\title{
Effectiveness of international service intelligence : a multifaceted perspective
}

Citation for published version (APA):

van Birgelen, M. J. H. (2000). Effectiveness of international service intelligence : a multifaceted perspective. [Doctoral Thesis, Maastricht University]. Universiteit Maastricht. https://doi.org/10.26481/dis.20001220mb

Document status and date:

Published: 01/01/2000

DOI:

10.26481/dis.20001220mb

Document Version:

Publisher's PDF, also known as Version of record

\section{Please check the document version of this publication:}

- A submitted manuscript is the version of the article upon submission and before peer-review. There can be important differences between the submitted version and the official published version of record.

People interested in the research are advised to contact the author for the final version of the publication, or visit the DOI to the publisher's website.

- The final author version and the galley proof are versions of the publication after peer review.

- The final published version features the final layout of the paper including the volume, issue and page numbers.

Link to publication

\footnotetext{
General rights rights.

- You may freely distribute the URL identifying the publication in the public portal. please follow below link for the End User Agreement:

www.umlib.nl/taverne-license

Take down policy

If you believe that this document breaches copyright please contact us at:

repository@maastrichtuniversity.nl

providing details and we will investigate your claim.
}

Copyright and moral rights for the publications made accessible in the public portal are retained by the authors and/or other copyright owners and it is a condition of accessing publications that users recognise and abide by the legal requirements associated with these

- Users may download and print one copy of any publication from the public portal for the purpose of private study or research.

- You may not further distribute the material or use it for any profit-making activity or commercial gain

If the publication is distributed under the terms of Article $25 \mathrm{fa}$ of the Dutch Copyright Act, indicated by the "Taverne" license above, 
Effectiveness of International Service Intelligence

A Multifaceted Perspective 
2000, Marcel J.H. van Birgelen, Maastricht.

All rights reserved. No part of this publication may be reprinted or utilized in any form or by any electronic, mechamical or other means, now known, or hereafter invented, including photocopying and recording, or in any information storage or retrieval system, without written permission from the copyright owner.

15BN: 90-9014408-0

Printed by: DATAWYSE, Maastricht. 


\title{
Effectiveness of International Service Intelligence
}

\author{
A Multifaceted Perspective
}

\section{PROEFSCHRIFT}

ter verkrijging van de graad van doctor aan de Universiteit Maastricht,

op gezag van de Rector Magnificus, Prof. Dr. A.C. Nieuwenhuijzen Kruseman, volgens het besluit van het College van Decanen,

in het openbaar te verdedigen

op woensdag 20 december 2000 om 16:00 uur

door

Marcel J.H. van Birgelen 
Promotor:

Prof. Dr.J.C. de Ruyter

Compromotor:

Dr. M.G.M. Wetzels

Beoordelingscommissie:

Prof. Dr. I.G.A.M. Lemmink (voorzitter)

Prof. Dr. S.J. Maijoor

Prof. T.A. Swartz, Ph.D. (California Polytechnic State University, San Luis Obispo, USA) 
The Information Sea is unstable. The hypotheses and intentions which emerge from it are complex and multifaceted.

(Baxter 1995)

What we observe is not nature itself, but nature exposed to our method of questioning.

(Heisenberg 1958) 


\section{Preface and Acknowledgements}

In general, the preparation of a doctoral dissertation is considered an undertaking that involves extreme personal effort on behalf of the Ph.D. candidate writing the dissertation. However, after the three year's process of preparing and completing this dissertation, Ihave come to the conclusion that a successful dissertation can only arise from own personal input in combination with the help and assistance of many other persons surrounding the Ph.D. candidate. In this preface, I gratefully take the opportumity to thank all the persons who stand at the foundation of this dissertation and who provided me with the necessary motivation and stimulation to bring it to an end.

First of all, I sincerely would like to thank both my advisors, Ko de Ruyter and Martin Wetzels, for their very valuable support, input, and ideas. Already before actually finishing my education at Maastrïcht University, Ko motivated me to enter the world of academia. It started five years ago with his enthusiastic supervision of my Master's Thesis and after its completion by giving me the opportunity to become one of his research assistants. After that, a position as junior lecturer paved the path to becoming Ko's Ph. D. student. During these last years, I have got to learn Ko as a person with inspiring academic and conceptual knowledge, which makes him a true seer especially in the area of services and interactive marketing. At times necessary, ko has always succeeded in providing me with the inspiration and motivation to carry on with the job. Especially during the final phase of finishing the dissertation, Ko's stimulation turned out to be extremely valuable and essential. For all this, I would like to say: Ko, thank youl

Silmultaneous to working with Ko, I also started to cooperate with Martin. Martin, by insiders known as Magic Marty, possesses the remarkable skills and insights that make him a wizard especially when it comes to statistics and numbers. At this point, 1 sincerely would like to thank him especilally for his willingness to assist me in this dissertation's analyses whenever requested. In addition, also a non-professional word of thanks should be expressed for the times Martin provided me with the possibility to stay over after spending a long night at the Irish pub, drinking our favorite Irish beer. Martin, slainte! Overall, my cooperation with Ko and Martin has resulted in various publications, which form a solid foundation to build a(n) (academic) career on.

Next, I would like to express my sincere gratitude to the members of the graduation committee, Prof. Dr. I.G.A.M. Lemmink, Prof. Dr. S.J. Maijoor, and Prof. T.A. Swartz, Ph.D. Thank you for the effort and time that you have put into reading the 
dissertation and for providing me with valuable remarks and suggestions. In its finalizing phase, these suggestions have made the dissertation to what it has become.

In addition, I gratefully wish to thank all my colleagues and former colleagues of the Department of Marketing and Marketing Research of Maastricht University. They created a professional environment that stimulated the accomplishment of this academic mission. Furthermore, the department needs to be thanked for providing the necessary financial and non-financial resources that have turned out to be invaluable for completing the dissertation. I hope our cooperation will continue to exist in the time to come. I would like to address a special word of gratitude to my room-mate Willemijn van Dolen. We spend quite some time discussing and helping each other with our work, talking about non-work-related matters, and, very important, laughing about a wide range of issues. Without that I certainly would have had a much harder time to reach the point where I am now. I consider myself fortunate to have had you as my roomy for the last three years! Finally, I would like to thank our department's secretaries, Mieke Donders and Pascalle Meuris, especially for their assistance in the finishing phase of the dissertation.

A grateful acknowledgement goes to the financial support that was given to me by the Faculty of Economics and Business Administration of Maastricht University and to the students who assisted me in collecting the data for the dissertation by writing their theses and conducting internships. Furthermore, I sincerely would like to thank our sponsoring organization, the frequently referred to multinational office equipment manufacturer, for their willingness to participate in the various research projects. Without them it would have remained virtually impossible to empirically test the conceptual frameworks and theoretical research hypotheses formullated.

The probably most fundamental expression of gratitude should go to my parents. They have always given me the opportunity and stimulation to learn and develop myself. They have always let me undertake the things I desired to do. Without them, this dissertation certainly would not have seen the light of day. Pap, mam, bedankt!

Finally, I would like to thank my partner in life, Lyan, with utmost sincerity. Her love, support, inspiration, and imfinite belief in me have driven me to finish this dissertation. Some day, in some way, I hope I will be able do the same for you. Lyan, thank you from the bottom of my heart! 


\section{Table of Contents}

Preface and Acknowledgements

1 Introduction. 1

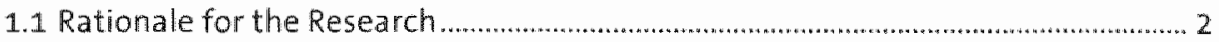

1.2 Rationale for the Research Domain ......................................................................... 3

1.3 Purpose and Research Questions............................................................................... 4

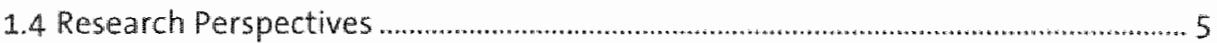

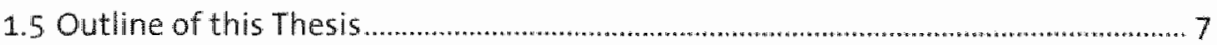

2 Culture's Consequences for International Service Intelligence............. 9

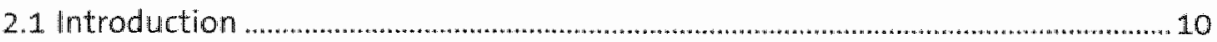

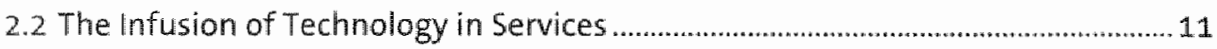

2.3 Culture's Consequences for Services ............................................................... 13

2.4 Development of Hypotheses .................................................................................. 14

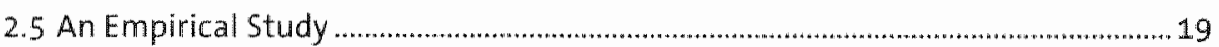

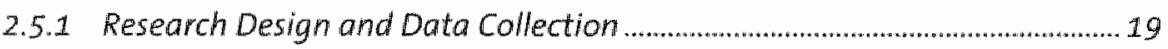

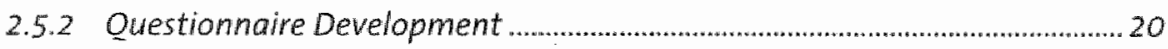

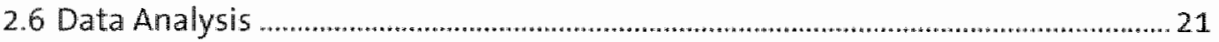

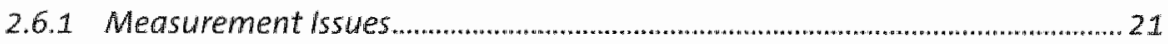

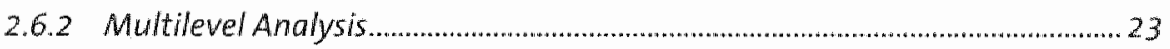

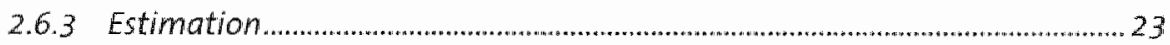

2.6.4 Model Building …............................................................................................ 23

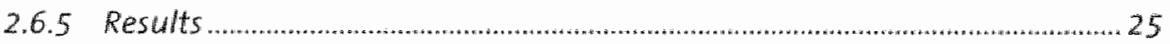

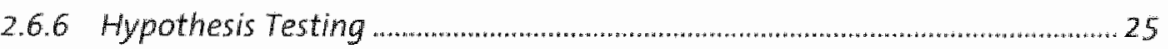

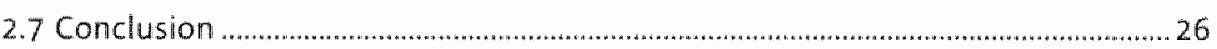

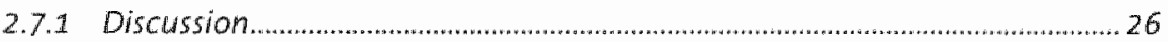

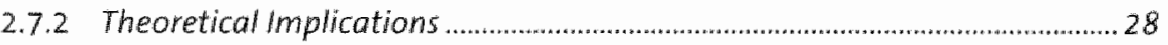

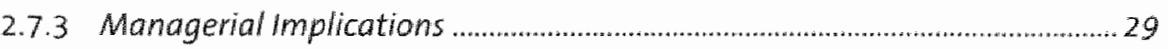

\section{The Impact of Incomplete Information on the Use of International} Service Intelligence............................................................................... 31

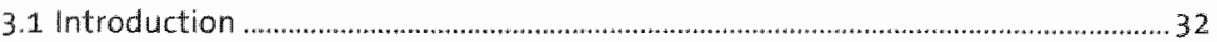

3.2 Uncertainty, Missing Information, and Marketing Decision-Making .................... 33

3.3 Informational Topics for Decision-Making in International Service Settings...... 35

3.3.1 Information on Cross-national Comparability of Research Data..................36 


\subsubsection{Information on Financial Accountability of international Service} Inverstments.

3.4. Developing Hypotheses on Incomplete information in Intermational Service

Settings.

3.5 An Experimental Study

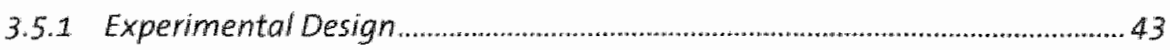

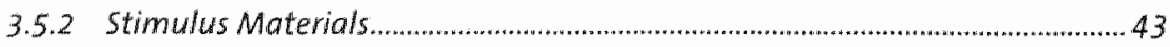

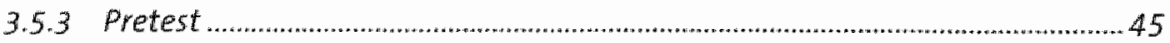

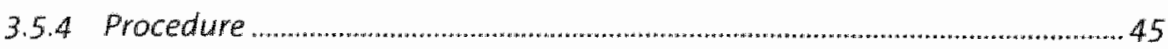

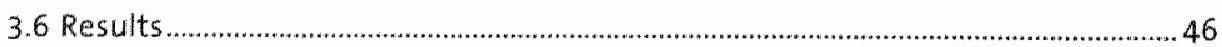

3.6.1 Manipulation Checks..................................................................................... 46

3.6 .2 Validity and Reliability of Dependent Measures ................................................ 47

3.6.3 Testing Assumptions of MANOVA and Dependent Variables .......................... 48

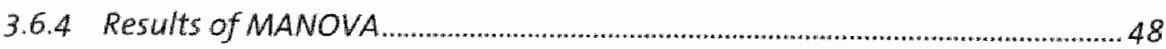

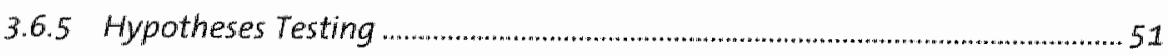

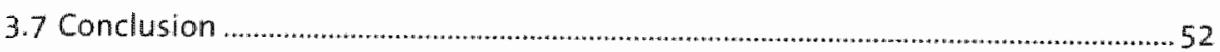

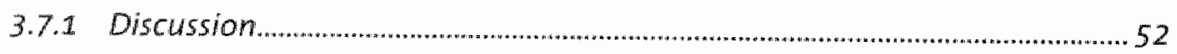

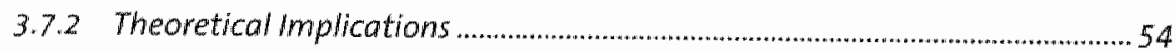

3.7 .3 Managerial Implications .........................................................................................5. 55

4 Effectiveness of International Service Intelligence: A Relational Perspective

4.1. Introduction

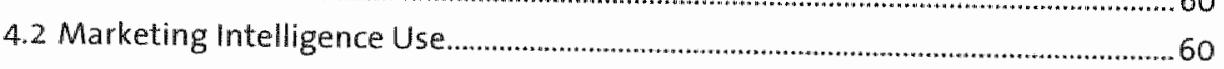

4.3 Development of a Conceptual Framework and Research Hypotheses ...................62

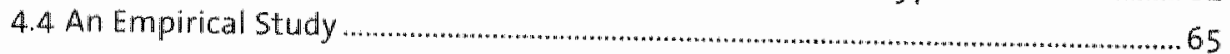

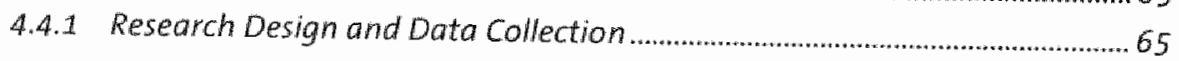

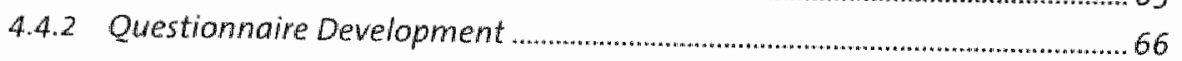

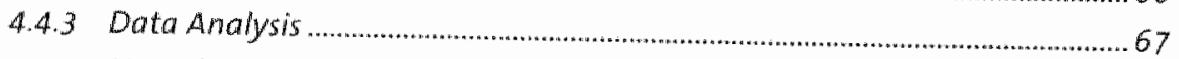

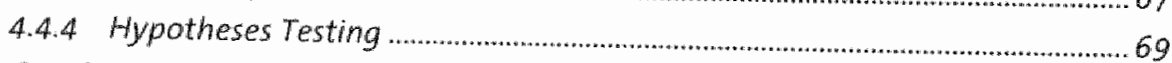

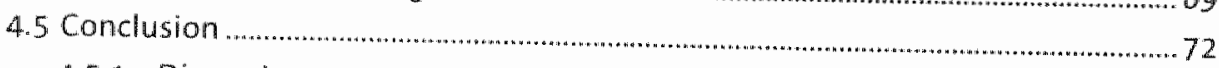

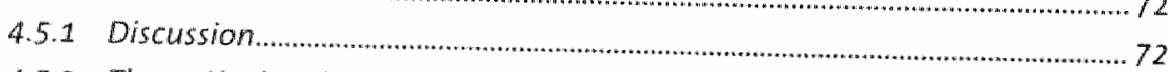

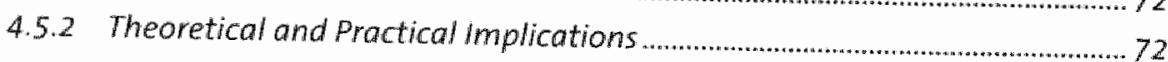

\section{The Impact of Attitude Strength on International Service} Intelligence Effectiveness.

5.1 Introduction

5.2 Attitude Strength: An Overview

5.3 Attitude Strength: Cognitive and Behavioral Consequences 
5.4 Conceptual Framework and Research Hypotheses ....................................................80

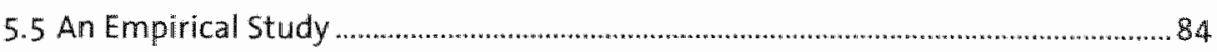

5.5.1 Research Design and Data Collection .................................................................84

5.5 .2 Questionnaire Development ............................................................................... 84

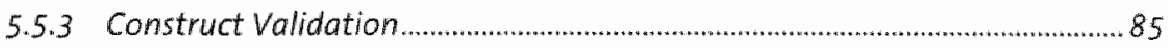

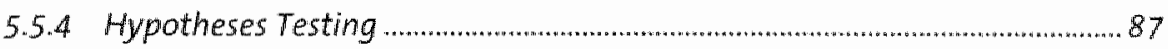

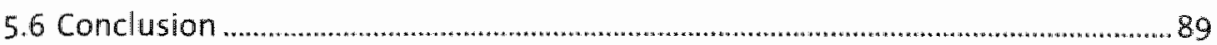

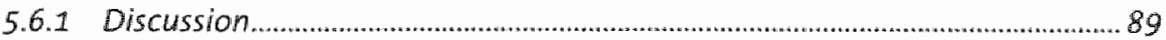

5.6 .2 Theoretical Implications ................................................................................ 91

5.6.3 Managerialimplications ................................................................................ 92

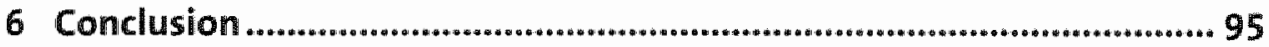

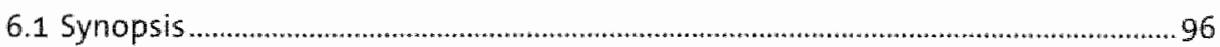

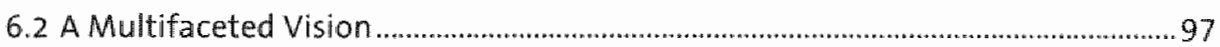

6.3 A Perspective on Future Research .............................................................................101

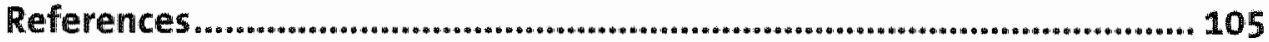

Appendix I: Modeling Hierarchical Relationships ...................................... 122

Appendix II: Sample Scenario ............................................................... 124

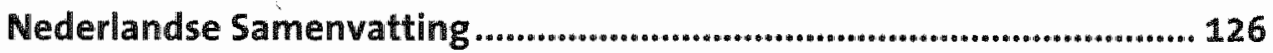

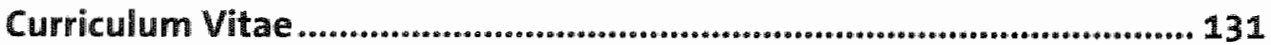



Introduction

1

Chapter 1

Introduction 


\section{Introduction}

\subsection{Rationale for the Research}

Whether the great $16^{\text {th }}$ century thinker Sir Francis Baker was a marketer avantwa-lettre we will never know, for we just do not have information to draw such a bold conclusion, and for making (marketing) decisions, information frequently forms a necessary input. However, Baker"s well-known adagio "for knowledge itself is power" seems more true than it ever was in today's information society. For present-day companies, marketing knowledge is of the utmost importance. More recently, various modern marketing thinkers have emphasized that marketing knowledge or intelligence can be used as a resource for beneficial actions towards customers, the organization, and even society as a whole (Barabba 8. Zaltman 1991). Intelligence has been defined as a complex and multifaceted concept (Merriam-Webster Dictionary 1997). In these definitions the ability to utilize knowledge in order to react to one's environment is the crucial element (Forrest 1999). More specifically, Menon and Varadarajan (1992, p. 53) suggest that a more effective use of marketing intelligence can be viewed as "critical to being more market-oriented and to succeeding in an intensely competitive business enviromment". Such conventional wisdom holds true for companies that operate in national markets and even more so for companies that deliver services internationally (Cowell 1993). The globalization of the service domain is increasing at rapid pace, blowing up the impact of llevels of uncertainty, ambiguity, and dynamism commonly associated with international markets (Deshpandé 1989). In order to deal effectively with these issues and to be able to exploit the power of knowledge as a competitive edge, there is a fundamental need for valid and reliable international service marketing intelligence (Czinkota \& Ronkainen 1995). More and more, however, marketing decision-making is hindered by the recurring phenomenon of informational overload, as rapid advances in information technology and research techniques are being made in the arena of international marketing research. Given the high costs of conducting marketing research and the opportunity costs of not using marketing intelligence, it seems important to pay attention to the issue of intelligence effectiveness in international services marketing. This is the mission of this thesis.

Albeit a necessary condition, information availability per se is not sufficient. To reap the fruits from international marketing information, it should actually be used by marketing as well as other decision-makers within the firm. Or, to use another metaphor, the proof of the pudding is in the eating. This is our conceptualization of 
information effectiveness. As indicated above, we adhere to the conceptualization of intelligence as a multifaceted concept. Therefore, the basic premise taken in this thesis is that the study of marketing information effectiveness in an international services context requires a multifaceted perspective. Our theory of perspectivism, firmly rooted in post-modernity, challenges the notion of scientific reality as linear and objective and adopts the notion of a systematic and participatory point of view. We need to pay attention to aspects of information, as well as the characteristics of both the actors who supply the information and the actors who need it as input for actual marketing decision-making, if we want to address the effectiveness issue effectively. This chapter sets the stage for studying these actors as well as the information that is shared between them. In other words, we will explain how we plan to develop a vision on the basis of our mission. The first chapter is structured as follows. Section 1.2 will give a more detailed justification of the main setting of this thesis, i.e. effective intelligence use in international service organizations. Subsequently, the general as well as the specific research questions addressed in this thesis are introduced. Next, section 1.4 briefly describes the research perspectives taken to answer these questions. Finally. section 1.5 provides an outline of the remainder of the thesis.

\subsection{Rationale for the Research Domain}

Nowadays, a growing number of service organizations are starting to broaden their horizons and explore the opportunities and challenges the international environment has to offer. This is caused by a variety of factors (Czinkota \& Ronkainen 1995). Firms can now expand their service activities far beyond their domestic markets and benefit from reaching many more customers due to the many advances made in information and communication technologies. As a result, market saturation in the home market can be avoided by lengthening or rejuvenating service life cycles in other countries. Furthermore, as markets open up, foreign policies that once were inflexible suddenly become variable. Subsidiaries can now be transferred from one country to another and necessary suppliers can be found on every continent. Cooperative agreements can be formed that enable all parties to bring their major strengths to the table and emerge with better services and ideas than they could produce on their own. In addition, research has found that multinational corporations face a lower probability of insolvency and less average risk than do domestic companies (Shaked 1986). Overall. international marketing not only provides service companies with new opportunities 
It also enables consumers all over the world to find greater varieties of services at lower prices and to improwe their life-styles and comfort.

The complexty of international service markets creates a need for international marketing research that can be used for solving strategic and tactical marketing problems. Cross-cultural differences between countries and the unfamiliarity with new markets make that urgent is sues such as what advertising to use across countries, whether and how to launch new services abroad, what prices to charge, and what foreign distribution channels to use should be paid explicit attention to. Furthermore, information is needed to monitor international customer satisfaction levels and changing trends in the environment, so that strategies can be evaluated with respect to their effectiveness and, whenever necessary, adapted accordingly. Finally, the investments required to meet the challenges of the internationalization factor are huge. There is a growing need for information on the accountability of these investments (Rust et al. 2000).

Issues of complexity seem to apply particularly to the international services domain, due to its unique qualities. Services are characterized by a high level of perceved purchase risk (Bateson 1995), especially when they are being provided by foreign, often unknown service providers (de Ruyter et al. 1998). This is caused by limited possibilities for (international) standardization (Guseman 1981), a strong focus on experience and credence attributes (Parasuraman et al. 1985), and high customer participation in the service production process (Bateson 1995). Related to this last issue is the fact that service encounters traditionally involve relationships between persons (Bitner 1990; Parasuraman et al. 1985; Shostack 1985). Since national culture is important for the formation of attitudes and preferences (Lovelock \& Yip 1996) culturally-determined norms and values held by the persons involved will affect service encounters in international environments (Usumier 1996). Cultural background can thus be expected to shape subjective expectations of service delivery that will have an effect on customer service performance perceptions and evaluations. As a consequence, research intelligence use in the international services domain represents a challenging area for further investigation.

\subsection{Purpose and Research Questions}

The overall purpose of this thesis is to gain an in -depth insight into how effective marketing intelligence can be gemerated for marketing decision-making in 
international service organizations. More specifically, the main research question can be formulated as follows:

\section{Which factors contribute to the effectiveness of marketing research intelligence in international service organizations?}

In order to address this issue effectively from a variety of perspectives, we formulated four related research projects. In the specific research projects the following research questions were posed:

1) How can cross-cultural variation be taken into account in international services research?

2) How do additional information attributes contribute to effectiveness of international service intelligence?

3) What researcher characteristics contribute to effectiveness of international service intelligence?

4) What user characteristics contribute to effectiveness of international service intelligence?

These research questions imply multiple research perspectives. In the next section, we elaborate on them.

\subsection{Research Perspectives}

Several research perspectives are taken in order to answer the main problem statement of this thesis in a comprehensive manner. First of all, we focus on characteristics or attributes of information. This intelligence attribute perspective examines characteristics of information and how they relate to information utilization by decision-makers in international service organizations. Such an approach has been frequently used in the academic marketing literature. Examples of intelligence attributes that have been identified are content quality, form quality, actionability, and acceptability (e.g., Deshpandé \& Zaltman 1982, 1984; Kohli \& Jaworski 1990; Menon \& Varadarajan 1992). In addition, previous research has focused on the impact of cross-cultural variation on information (e.g., Donthu \& Yoo 1998; Furrer et al. 2000; Hofstede 1980; Mullen 1995; 5tyles 1998) and financial accountability (Buzzell et al. 1975: Rust et al. 1995) and its potential impact on managerial decision-making. 
Moreover, in addition to the presence of intelligence attributes, a substantial body of research has also looked at the absence of certain attributes and its impact on decision-maker utilization (e.g., Jagacinski 1991; Kahneman et al. 1982; Lim \& Kim 1992; Lipshitz \& Strauss 1997).

In addition to the intelligence attribute perspective, it seems warranted to pay attention to the context within which the information is presented. In order to firmly anchor our thesis in the services research domain, we position intelligence effectiveness in the context of the intelligence service encounter. We term this perspective the relational exchange perspective. The basic premise taken is that the provision of intelligence is essentially a relational exchange between a service provider (e.g., the researcher) and a client (e.g., the user or decision-maker). Therefore, in addition to studying the quality of the service provided, we feel that the focus should also be on the encounter or interaction between provider and customer. Previous research has focused mainly on user perceptions of the interaction (e.g, Barabba 1978; Ernst 1976), trust, and characteristics of the intelligence provider on user evaluative judgments (e.g., Moorman \& Austin 1995; Moorman et al. 1992, 1993). In this study. we adopt a similar point of view and look at the impact of decision-maker perceptions of the service and the service provider on effectiveness of service intelligence.

A third perspective taken in this thesis focuses on the viewpoint or internal attribution by users of information and its impact on service intelligence effectiveness. We term this the attitudinal perspective, as it is oriented towards the impact of user attitudes on cognition and behavior. This is an approach that has been taken mainly in the area of social psychology (e.g., Chaiken et al. 1995; Eagly \& Chaiken 1993, 1995; Krosnick et al. 1993; Pomerantz et al. 1995). However, we believe that it holds promising potential for explaining service intelligence effectiveness in an international context too. We argue that ultimately the use of information also depends on a conducive user attitude.

\section{Figure 1-1: The Intelligence Service Encounter}

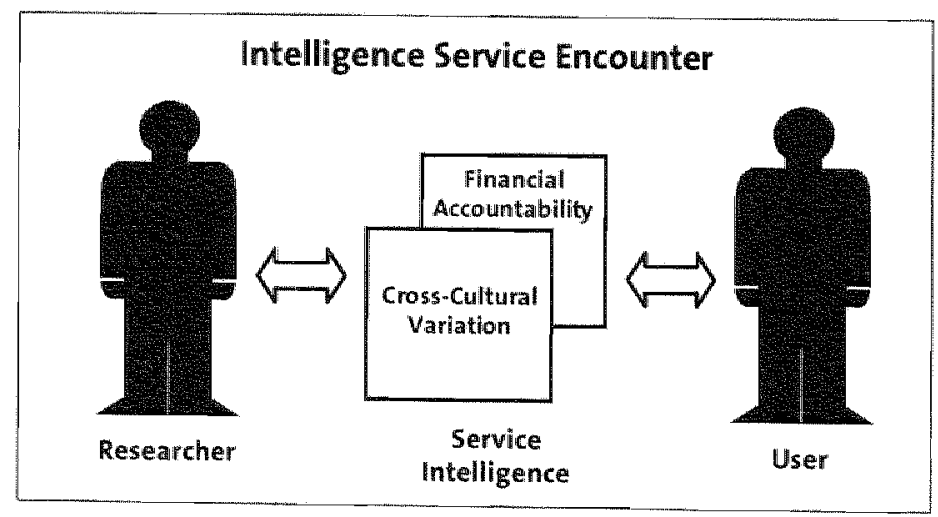


Figure 1-1 visualizes the multifaceted approach aimed at developing our vision of effectiveness of international service intelligence. Having explained our point of departure, we will now outline where our journey will take us in the next section.

\subsection{Outline of this Thesis}

The main body of this thesis consists of four chapters, each of which incorporate both a conceptual development and an empirical research orientation, as well as a theoretical and managerial implications section. Essentially, this thesis contains a collection of four related research projects, as we observed above. In each chapter, we attempt to contribute to addressing aforementioned research questions. First, we relate the content of the chapters to our multifaceted approach. In chapters 2 and 3 , the focus is on marketing intelligence attributes. This perspective will be supplemented in chapter 4 by taking user perceived intelligence provider characteristics into consideration. In addition to the quality of content, user's perception of the researcher are taken into account in an attempt to explain intelligence effectiveness, i.e. the likelihood of actual information use. In chapter 5, finally, the focus is on the user of information in accordance with the attitudinal perspective.

Secondly, we briefly introduce the content of the chapter in relation the research questions. Chapter 2 relates to the first research question. In the chapter, we address the impact of cross-cultural variation on customer evaluations of various modes of service delivery. We attempt to demonstrate how cultural background characteristics of customers can nuance international intelligence on service performance.

Chopter 3 reports on a study that was undertaken to investigate the impact of various types of intelligence in international service research. Here, we take the absence of aforementioned types of information into account. By means of an experimental design we examine the effect of two types of incomplete information on decision-maker utilization of marketing intelligence. A distinction is made between (I) information on cross-cultural comparability of international marketing research intelligence and (11) information on the financiall accountability of tactical actions that can be undertaken on the basis of a specific research project in an assessment of decision-maker preference.

Chapter 4 supplements the focus on properties of research information by taking user perceived evaluations of the intelligence provider into consideration. A study was designed to simultaneously assess the impact of intelligence attributes as well as 
researcher characteristics on intelligence use by decision-makers in an international service firm.

In chopter 5 , the focus is on the attitude of a decision-maker who is supposed to use international service research results. We attempt to explain marketing intelligence use as a function of a decision-maker's attitude towards the subject of information (in this case, international customer satisfaction).

Finally, chapter 6 combines the results of aforementioned studies by developing a vision that addresses our mission. 


\section{Chapter 2}

\section{Culture's Consequences for International Service Intelligence}

in chapter 2, the focus is an attributes of international service intelligence. In the chapter a perspective is introduced which aims at addressing the impact of crosscultural variation on service evaluation intelligence with respect to three different service delivery modes. As a result of recent technological advances, the services partfolio of many companies is extended from the traditional, face-to-face service encounter towards voice-fo-voice and bit-ta-bit technology-based services Mare specifically, we examine the interplay between perceived service performance and customers' cultural background in generating international intelligence on satisfaction judgments for traditional vs. technology-based after-sales services. Results suggest that culture moderates the service quality-customer satisfaction relationship for technology-based after-sales serwices in contrast to face-to-face service encounters. Consequently, cross-cultural variation forms an impontant attribute of international service intelligence. 


\section{Culture's Consequences for International Service Intelligence}

\subsection{Introduction}

It has been widely recognized that delivering superior service quality is a key factor in creating a viable competitive advantage (Rust 1998; Zeithaml 2000). In the past decades, therefore, a great deal of research attention has been devoted to customer evaluations of their encounters with service providers (e.g., Bitner 1990; Cronin \& Taylor 1992; Oliver 1993; Parasuraman et al. 1985). In these studies the focus has been almost exclusively on the personal, face-to-face interaction between customers and frontline service employees and the service encounter has been conceptualized as "high-touch, low-tech" "Bitner et al. 2000). However, rapid advances in technology are fundamentally transforming and changing the nature of customer-company interactions (Rust 1999). In addition to the traditional service delivery format (face-toface), companies are now using voice-to-voice (e.g, toll-free telephone support) and bit-to-bit (e.g., online service delivery) modes, as well as combinations thereof, to deliver their services. This has enabled firms to expand the notion of competitive service positioning and take the commonplace wisdom of "listening to the voice of the customer" as well as the long-standing concept of "the customer as partial employee" literally.

In addition, the infusion of technology in service delivery is leading to a process of spatial convergence, as a result of which service delivery can now easily transcend geographical as well as cultural boundaries. Interestingly, many firms treat spatial convergence synonymously with cultural convergence in their communications. strategy. This becomes clear, for instance, from the commercials by ICT Infrastructure provider CISCO Systems in which people from various cultural backgrounds are asking television viewers around the globe enthusiastically "are you ready?". Technology provider Lucent is telling its customers to "expect great things" in the pay-off line of their recent advertising campaign. The underlying assumption here is the inevitability of a world-wide and pan-cultural acceptance and positive affirmation of information technology. However, it remains disputable whether or not the adoption and evaluation of technology is culturally neutral.

In this chapter, we argue that, particularly for intelligence on evaluations of customer-company service interactions infused with technology, culture's consequences should not be underestimated. We concur with the view that even in borderless cyberspace "there are very significant borders, such as cultural ones" (Brace 
2000, p. 70). Our objective is to examine the interplay between perceived service performance and dimensions of customer's cultural backgrounds in the formation of intelligence on evaluative judgments with regards to traditional vs. technology-based service delivery formats. The chapter is structured as follows. First, we discuss the impact of companies' use of technology in serving customers. Subsequently, we discuss the relationship between culture and service evaluations and we develop a set of hypotheses that focus on the moderating effect of cultural characteristics on the formation of overall customer satisfaction with the after-salles service delivery. Next, we report the results of a large-scale empirical study aimed at testing these hypotheses. In conclusion, we discuss the theoretical and managerial implications of our findings.

\subsection{The Infusion of Technology in Services}

Faced by the challenges of intelligent interactivity, many service providers have supplemented their traditionall face-to-face service delivery format with services that involve multiple media. In addition to interpersonal service encounters, customers can now opt for technology-mediated interactions for service delivery, varying from voice technology to fully automated self-service-aids (Risch Rodie \& Schultz Kleine 2000). The infusion of technology is rapidly and fundamentally changing the nature of the service encounter with far-reaching consequences for companies as well as customers. It has been argued that the increasingly significant role of technology in service delivery necessitates a re-conceptualization of established concepts in the services marketing research domain. This has lead Parasuraman (1996) to conclude that the well known services marketing triangle (Bitner 1995; Kotler \& Arrnstrong 1997), symbolizing the tripartite relationship between customers, employees and service company should be enhanced by the inclusion of technology as an additional dimension in order to reflect the present complexity inherent in service delivery. As a consequence, service encounters are now conceptualized as "the dynamic relationship between employees, customers, and technology" (Bitner et al. 2000, p. 141) and service encounters can be classified according to their technology-based delivery mode (Dabholkar 1994).

The implementation of technology in the delivery of services offers a wide range of advantages both to service providers and customers. Increased opportunities for service customization and flexibility, service recovery, and spontaneous customer delight have been identified as the main three drivers of customer satisfaction to be 
influenced by the infusion of technology (Bitner et al. 2000). Especially, customization and flexibility have emerged as important consequences of technology in services (Fisher 1998; Peppers \& Rogers 1997; Quinn 1996). Other advantages frequently mentioned are the possibility to develop multichannel service strategies, the establishment of more effective informational exchange, higher consistency in the delivery of standardized services, lower costs, and increase in customer choice (Heim \& Sinha 2000). In research on customer evaluations of service delivery technology, these advantages feature prominently in attribute-based models (Dabholkar 1996; De Ruyter et al. 2000\%.

More fundamentally, however, customer evaluative judgments of technology based services may also be dependent on the nature of the customer's role in the service delivery process (Dabholkar 2000). In traditional after-sales service delivery, $a$ service employee could visit a customer's site for servicing or repairing equipment ('doit-for-me'). The customer's role remains fairly passive and customer participation is low. As a consequence, the well-known service quality performance variable will be the main determinant of customer satisfaction (Rust \& Oliver 1994). With the advent of technology-based service delivery modes, however, customers are more actively involved in the service delivery process. The service can now be initiated and delivered by the customer without direct or indirect face-to-face or voice-to-voice contact with service representatives ("do-it-yourself) (Barnes et al. 2000). In banking, travel, brokerage and many other service and goods ind ustries successful self-service business models have already been developed. Due to a higher degree of customer participation, customer satisfaction evaluations may be increasingly based on the customer's attitude towards the service technology used. Attitude-type variables, such as customer technomreceptivity (Barnes et al. 20c0), perceived media richness and symbolism (Treviño et al. 2000) and the disposition towards self-service technology (Dabholkar 1996) also have been demonstrated to have an impact on overall service evaluations. These findings are in line with previous research that has identified the impact of a generalized attitude in relation to technology evaluations in other contexts (Dickerson \& Gentry 1983; Ledingham 1984), as well as studies on social influence and media use (Fulk 1993; Schmitz \& Fulk 1991; Fulk \& Boyd 1991). Consequentially, it seems important to take individual and/or situational context variables into account in explaining the formation of customer evaluations of the widening scope of service delivery formats. To date, there is an accumulating body of academic research on the role of individual differences in evaluations of technology-based services, resulting in important nuances in customer profiles (Dabholkar 1996; Meuter et al. 2000). In contrast, less is known about the impact of contextual factors with regards to customer evaluations of technology-based service encounters (Parasuraman 2000). 
Yet, there is a compelling rationale for examining the role of cultural background characteristics in relation to customer evaluations of services. In the managerial literature it has been emphasized frequently that the infusion of technology in service delivery is leading to a collapse of temporal and spatial distance (Maney 1997). It is argued that technology enables a $24 / 7$ service delivery, worldwide, and independent of geographic location. Online presence is often accompanied by offline facilities like contact centers providing 24 hours support to customers worldwide resulting in an advanced convergence of voice and data (Richardson \& Marshall 1999) and panregional distribution centers for automated, instantaneous ordering and shipping of supplies to clusters of geo-markets (e.g., Hewlett-Packard's European service operation). As a consequence, services are increasingly being delivered to customers from various cultural backgrounds (Huber et al. 1997). However, despite the ephemera of cyberspace it has been stated that "technology is not independent of culture" (Trillo 1997). It has been argued that the cultural context, expressed in shared norm and value systems (Hof stede 1980; Kluckhohn 1951), has an impact on the acceptance of technology-based interactions between people (Trillo 1997). Likewise, in a business environment that is increasingly characterized by cultural diversity, divergent identification systems will influence acceptance and evaluation of technology-based service delivery (Steinman et al. 2000). Therefore, we posit that cultural contingencies. are expected to moderate the formation of customer evaluations of services and subsequently have an impact on the validity and rellability of service intelligence. ln the next sections we will explain how.

\subsection{Culture's Consequences for Services}

In addition to the infusion of technology, it seems important to consider culture's consequences for services and services intelligence. Whether mediated by technology or not, services involve interactions and relationships between customers and service providers (Bitner 1990; Parasuraman et al. 1985; Shostack 1985). Therefore, Usunier (1996, p. 252) argues that "prevailing cultural norms will apply in service encounters as they apply in any social interaction". Gallois and Callan $(1997$, p. 86) even state that all interactions between people are "governed by culture-specific social rules". Culture, described as a collective value system shared by a category of people (Hofstede 1991), influences the formation of attitudes and preferences (Lovelock \& Yip 1996). Culture manifests itself in non rational values programmed early in people's lives (Hofstede 1980). According to Oliver (1997) and Gallois and Callan (1997), these values are 
predisposing conditions for desires as well as the social rules of interaction. As such, they shape subjective attitudes and preferences and hence form the basis for comparison standards customers use for evaluating services. Furthermore, as social networks of customers are expanding beyond their own cultural horizons (e.g., through electronic multiuser groups), customer expectations and perceptions of service delivery are increasingly shaped by intercultural connectivity.

This becomes clear from a number of recent studies that focused on culture's consequences for services in which the relationship between Hofstede's (1980) dimensions of culture and service evaluation formation processes is examined empirically. Donthu and Yoo (1998) found empirical evidence for effects of cultural traits on service quality expectations. Low power distance consumers, for example, tend to have higher overall service quality expectations and expect service providers to be responsive and reliable. Correspondingly, Furrer et all. (2000) conclude that high power distance customers find reliability and responsiveness less important. Individualistic consumers also were found to have higher overall quality expectations, but expect the service provider to be more empathic and assuring (Donthu \& Yoo 1998). Mattila (1999) reports that customers from different cultures tend to value various service elements differentially. She concludes that Western customers, in contrast to Asians, rely more on tangible cues from the physical environment as a result of lower power distance levels.

However, the empirical results with respect to the relationship between culture and service evaluations seem far from univocal. In contrast to Mattila (1999), Furrer et al. (2000), for example, report a strong positive relationship between power distance and relative importance of tangible service elements. This leads us to conclude that the theoretical and empirical foundation of culture's consequences for services is still rather fluid and that more research on the interrelation between culture and services performance evaluation is needed, particularly given the complexities of technologymediated cross-cultural service delivery. In the next section, therefore, we develop a number of hypotheses that focus on the moderating impact of Hofstede's cultural dimensions on service evaluation formations by customers.

\subsection{Development of Hypotheses}

The concepts of service quality and customer satisfaction have featured prominently in the academic services literature of the past two decades. One issue that has given rise to considerable debate is the sequential order of service quality and satisfaction. 
Recent research has convincingly argued and empirically demonstrated that satisfaction should be viewed as the superordinate construct (e.g., De Ruyter at at. 1997; Dabholkar et al. 2000). In other words, there is growing consensus that service quality is an antecedent to satisfaction with services. In adherence to this perspective, we examine the impact of perceived service quality with traditional and technologybased service delivery mades on overall customer satisfaction. More specifically, we make a distinction between voice-to-voice or call center-based service support and bitto-bit or electronic (web-based) service delivery. In an after-sales service setting, voice. to-voice service delivery is a format in which there is real-time, personal telephone interaction for supplying information and/or customer coaching by a service employee to perform self-service (e.g., solving product problems). Electronic service delivery reflects online self-service via the Internet. Furthermore, we explore how the service quality-satisfaction relationship for the various service delivery modes is moderated by dimensions of national culture.

Based on the line of argument in the preceding sections of this chapter, our premise is that culturally-determined variation in attitudes and preferences is likely to cause customers from various cultural backgrounds to differentially evaluate service delivery modes, depending on the type of technology infusion. In methodological terms, the main effects in our model pertain to the effect of perceived service quality on overall customer satisfaction, while interaction effects relate to the moderating effect of culture on the service quality-customer satisfaction relationship. Due to the fact that conceptual as well as empirical evidence for the impact of cultural dimensions on customer evaluative judgments is non-existent and that it is consequentially virtually impossible to specify a-priori relationships, we will not focus on the main effects of cultural dimensions on overall customer satisfaction with the service delivery in this chapter. Moreover, our premise is that both voice and electronic service delivery involve sophisticated implementation of technology and that, therefore, it is not warranted to make a-priori distinctions between these two technology based service delivery modes.

With regards to the main effects of perceived service quality on overall customer satisfaction, we hypothesize a positive relationship for all three service delivery modes on the basis of aforementioned previous research:

$H_{1}$ : Both for traditional (face-to-face) and technology-based (voice-to-voice and bitto-bit) after-sales senvice delivery modes there will be a positive relationship between perceived service quality and overall customer satisfaction. 
It has been argued that in comparison to traditional, face-to-face service delivery modes, technology-based services entail higher levels of active customer participation or self-service (Barnes et al. 2000; Bitner et al. 2000; Dabholkar 2000) and that a more active involvement of the customer in the service delivery process has a positive impact on service evaluations (Rish Rodie \& Schulz Kleine 2000). As a consequence, culturally determined norm and value systems will be more prevalent in service evaluations by customers. Furthermore, due to location and time constraints, face-toface services are commonly delivered lacally, i.e. by service employees from the same cultural background. In contrast, we argued that technology-based services are frequently not hindered by these constraints and that they may be delivered across cultural boundaries, invalwing interactions between people with different cultural backgrounds. Therefore, we posit that for the face-to-face delivery format, cultural dimensions will not have an impact on the service quality-satisfaction relationship:

$H_{2}$ : National cultural dimensions will not moderate the positive effect of perceived service quality on overall customer satisfaction in case of face-to-face after-sales service delivery.

Alternatively, we expect that for techmology-based service delivery modes (voiceto-voice' and 'bituto-bit') national culture does moderate the service qualitysatisfaction relationship. In the remainder of this section, we will nuance this position by making a case for the interaction effects of four cultural dimensions discerned by Hofstede (1980) on the formation of a customer's evaluative judgment with respect to service delivery modes.

To begin with, we focus on the power distance dimension. According to Hofstede (1991), in such cultures a certain distance between powerful and less influential people is expected and may even be desired. In a recents study, Furrer et al. (2000) conclude that customers in high power distance cultures focus more on tangible service aspects, rather than service employee behavior-related dimensions such as reliability and responsiveness. Since technology-based services are mediated and, therefore, the interaction between service employee and customer is more distant and involves a relatively high degree of tangible technology-related elements, we expect that in high power distance cultures perceived performance of an impersonal, technology-based service delivery mode can contribute to overall service satisfaction. Therefore, we posit that for technology-based service delivery modes the relationship between service quality and overall satisfaction will be moderated by the cullural dimension of power distance: 
$H_{3 a^{*}} \quad$ The positive effect of perceived voice-to-voice after-sales senice quality on overall customer satisfaction will be stronger when national cutural power distance is higher;

$H_{3 i}$ : The positive effect of perceived bit-to-bit after-sales service quality on overall customer satisfaction will be stronger when national cultural power distance is higher.

As we argued above, culture is reflected in its members' attitudes and predispositions (Lovelock \& Yip 1996). One relevant predisposition for the adoption and positive evaluation of new service delivery formats is innovativeness. Steenkamp et all. (1999) found effects of cultural characteristics on consumer innovativeness, i.e. the predisposition to buy new and different products and brands rather than to adhere to earlier choices and patterns of consumption. Consumers in countries characterized by higher individualism were found to be more innovative. In individualistic cultures initiation of new and unknown behaviors, independently of other persons, seems to be valued more. Persons in individualistic cultures seem to be more trusting and less risk averse in exchange relationships with external, unknown parties (Yamagishi \& Yamagishi 1994). In addition, persons with a lower need for interaction with service employees were found to display more positive attitudes towards using new computerized self-service options (Dabholkar 1992). It has been argued that individualists show less interest in interaction and can be bothered less by the social absence of a service provider in case of technology-based after-sales services (Gallois \& Callan 1997). Therefore, we expect that customers in more individualistic cultures will hold a more positive attitude towards innovative technology-infused service delivery modes and, therefore, that the perceved service quality-satisfaction relationship will be reinforced by the individualism dimension:

$H_{4:}$ : The positive effect of perceived vaice-to-woice after-sales service quality on overall customer satisfaction will be stronger when national cultural individualism is higher;

$H_{4 b}$. The positive effect of perceived bit-to-bit after-sales service quality on overall customer satisfaction will be stronger when national cultural individualism is higher.

Masculinity has also been found to have an effect on consumer inmovativeness (Steenkamp et al. 1999). In countries characterized by higher masculinity scores, consumers are more innovative, the rationale being that masculine cultures are more material- and achievement-oriented (Hofstede 1991). Purchasing new items or making 
use of new, innovative services may be considered a consequence of this tendency (Mowen 1995). Therefore, we posit that in more masculine cultures, innovative service delivery formats will be evaluated more positively. Consequently, we hypothesize that:

$H_{s w^{\prime}} \quad$ The positive effect of perceived vaice-to-voice after-sales service quality on averall customer satisfaction will be stronger whem national cultural masculinity is higher:

$H_{\text {sir. }}$ The positive effect of perceived bit-to-bit after-sales service quality on overall customer satisfaction will be stronger when national cultural masculinity is higher.

Finally, we argue that due to higher unfamiliarity with innovative, technologybased service delivery modes such services will be perceived as more risky. In accordance with Lynn and Gelb"s (1996) finding that penetration of technical durables is lower for higher levels of uncertainty avoidance, acceptance and utillization of services strongly relying on technology is likely to be lower in high uncertainty avoidance cultures. In such cultures, perceived quality of innovative service delivery modes is less likely to result in additional service satisfaction. Furthermore, as there is less room for contextual cues which may reduce ambiguity in interactions and hinder the formation of an evaluative judgment, we hypothesize that:

$H_{6 a n}:$ The positive effect of perceived voice-to-voice after-sales service quality on overall customer satisfaction will be weaker when national cultural uncertainty avoidance is higher:

$H_{6 b}$ The positive effect of perceived bit-to-bit after-sales service quality on overall customer satisfaction will be weaker when national cultural uncertainty avoidance is higher.

In the next section, we discuss the results of an empirical study conducted to test our research hypotheses. 


\subsection{An Empirical Study}

\subsubsection{Research Design and Data Collection}

We tested our hypotheses by conducting an empirical study with an international sample of customers of a multinational office equipment manufacturer, the headquarters of which are located in the Netherlands. For several years, the company has been conducting a large-scale international survey aimed at measuring overall customer satisfaction with after-sales services and support for its high-tech office equipment. The data that is used in the present study is data from this international survey and was collected in 1999 in eleven different countries, including the Netherlands, the United Kingdom, Norway, Austria, Germany, France, Sweden, Belgium, the United States, Spain, and lreland.

In this study, we focus on three distinct after-sales services delivery formats. The first format is the traditional service wisit, in which a service engineer delivers face-toface services (e.g., servicing or repairing equipment and installing supplies) at a customer's site. This format refers to what we have termed 'do-it-for-me-services' and can be characterized in terms of high-touch, low-tech (despite the often technical nature of the after-sales services). Typically, these services are rendered by a workforce of service engineers within each country. The technology-based after-salles service delivery modes are represented by the voice-to-voice and bit-to-bit services. In the callbased service delivery, the customer is transferred to a helpdesk via a semi-automated response system where helpdesk agents (qualified service engineers) register and help solving problems and provide answers to questions. Frequently, helpdesks serve multiple international markets. Both the customer and the service employees are actively involved in a 'do-it-together' service delivery process. The electronic service deliwery mode rellates to the facilitating process of ordering necessary supplies like toner, paper, replacements as well as diagnosing and repairing equipment online. Typically, this increasingly accurs through web-based services. This "do-it-yourself" service is predominantly initiated and controlled by the customer.

In total, 14,888 questionnaires were sent to customers in the eleven countries. Of these, 7740 usable questionnaires were returned, resulting in a relative response rate of $5.2 \%$. The absolute response rates per country were as follows; the Netherlands: 320 ; the United Kingdom: 739, Norway: 598, Austria: 194. Germany: 477. France: 1168, Sweden: 507, Belgium: 550, the United States: 2726, Spain: 374, and Ireland: 87 . The differences in country-level sample sizes reflect the relative size of the markets in each country. A more detailed profile of our sample emerged on the basis of the following 
background variables: respondents were active in various functions such as sales manager $(18 \%)$, operations manager $(41 \%)$, company buyer $(23 \%)$, and general manager (5\%). As a token of this study's relevance, $89 \%$ of the respondents considered information on the manufacturer's service levels important. Furthermore, at the time of the survey, $37 \%$ of the respondents also used other brands of office equipment from other suppliers.

\subsubsection{Questionnaire Development}

The items used to measure the quality of our three after-sales service delivery modes discussed above were part of a larger questionnaire administered in the international customer satisfaction survey. When conducting an international survey the establishment of construct equivalence is crucial, meaning that the underlying meaning and interpretation of the items included in the questionnaire should be equal across countries. For establishing construct equivalence a four-step procedure was followed. The first step consisted of conducting focus group interviews with managers of the lacal operating companies in order to generate ideas on what is important for measuring the quality of the after-sales services. Next, the preliminary questionnaire was developed by formulating quality measurement items on the basis of the focus group interviews and the dimensions of the well-known SERVQUAL instrument designed by Parasuraman et al. (1985). These items were formulated in an expectancy disconfirmation format in which customers could express their perception of performance relative to the expectations they hold (Cronin \& Taylor 1992). The questionnaire was double-back translated in each country in order to check translation quality (Brislin 1980). Third, interviews were being held with managers for evaluating the questionnaire. On the basis of comments given during these interviews some items were adapted. Finally, a quantitative pretest was conducted with smaller samples of customers in each country, which also resulted in some minor adaptations to the fimal questionnaire.

All quality items were measured on a 9-point scale, ranging from 1 "much worse than expected" to 9 "much better than expected". Service visit quality was operationalized by six items, e.g., "Ability of XXX's service technician to solve your problem in one visit", "Degree to which $X X X X^{\prime}$ 's service technician provides feedback on the progress of the service visit', and 'Competence of XXX's service technician'. The quality of the service call was measured by four items, e.g. 'Competence of $X X X$ 's call handling staff in registering your problem", "Understanding of your needs by $X X X$ 's call handling staff', and 'Feedback on when your problem will be solved'. Four items were also used to measure quallity of the electronic after-sales service. These items related 
for example to the accessibility of the electronic service, the information provided through the electronic system, and the responsiveness of the system. Finally, overall customer satisfaction with the manufacturer's after-sales services was measured by a 9-point single-item scale ranging from 1 "very dissatisfied' to 9 "very satisfied".

As recommended by Schwartz (1994) and in correspondence with Steenkamp et al. (1999) the cultural characteristics of the eleven countries participating in our study were represented by an update of the Hofstede (1980) ratings provided by Hoppe (1990). These extensively validated ratings can be considered a more contemporary measurement of national culture. Furthermore, the Hofstede dimensions have been successfully used in previous consumer-related research (Lynn et al. 1993; Roth 1995).

\subsection{Data Analysis}

\subsubsection{Measurement Issues}

Reliability analysis on the items indicated sufficient internal consistency in terms of Cronbach's alpha. Across the entire sample the $\alpha$ reliability coefficients for service visit, service call, and electronic service delivery were $0.96,0.93$, and 0.93 respectively. All scores well-exceed the recommended cut-off value of 0.70 (Nunnally \& Bernstein 1994). On a country level the coefficients ranged from 0.93 to 0.97 for the service visit, 0.88 to 0.95 for the service call, and 0.91 to 0.97 for the electronic service delivery.

in cross-national research aimed at empirically assessing and comparing theoretical frameworks measurement instruments first have to be evaluated with respect to their cross-national equivalence. Instruments cam only be compared when showing cross-national invariance. The underlying reasoning is that cross-national differences in scale means or structural relationships between scale scores then indicate true differences between countries, instead of being caused by systematic response biases or problems with scale artifacts, reliability, or nonequivalence (Mullen 1995; Steenkamp \& Baumgartner 1998; van den Vijver \& Leung 1997).

Structural equation modeling is the most widely used technique to assess measurement invariance cross-nationally. We tested for measurement invariance using a hierarchical ordering of nested models (Bollen 1989; Byrne et al. 1989; Jores kog 1971a; Marsh 1994; Marsh \& Hocewar 1985):

1. Totally non-invariant model with no between country invariance constraints;

2. Factor loadings invariant across countries; 
3. Factor loadings, factor correlations, and factor variances invariant across countries, and;

4. Totally invariant model with all parameters invariant across countries.

The first model essentially tests whether the pattern of salient and nonsalient factor loadings is equal across countries: this is referred to as configural invariance (Steenkamp \& Baumgartner 1998). Our results indicated that the data fit well with the à priori hypothesized model: $\chi^{2}(814)=1191.91$ (p<0.001); GFl=0.90; TLl=0.99; CFI=0.99; RMSEA $=0.031$. Although the $\chi^{2}$-statistic is significant and that is indicative of a poor fit, the other goodnes5-of-fit indices indicated a good fit. All factor loadings are significant and the standardized loadings exceeded 0.5 . In the second model we tested whether the factor loadings are equal across countries $\left(\Lambda^{1}=\Lambda^{2} \ldots=\Lambda^{11}\right)$; this is referred to as metric invariance (Steenkamp \& Baumgartner 1998). Our results indicated that the diata fit satisfactory with the à priori hypothesized model: $\chi^{2}(924)=1548.89(p<0.001)$; $C F|=0.86 ; T L I=0.98 ; C F|=0.98 ; R M S E A=0.038$. The increase in chi-square between the two models is significant: $\Delta \chi^{2}(110)=356.98 \quad(p<0.001)$. However, the remaining goodness-of-fit indices (TLU, CFI and RMSEA), which are less sensitive to sample size, show a less marked decrease in fit. In the third model we tested whether the factor loadings, factor correlations and factor variances are invariant across countries. Our results indicated that the data fit satisfactory with the a priori hypothesized model: $\Delta \chi^{2}(984)=1687.57$ ( $\left.p<0.01\right) ; G F=0.85 ; T L I=0.98 ; C F l=0.98 ; R M S E A=0.037$. The increase in chi-square between the two models is significant: $\Delta \chi^{2}(60)=138.68$ ( $\left.p<0.001\right)$. However, the remaining goodness-of fit indices ( $T L I, C F I$ and RMSEA), which are less sensitive to sample size, show a less substantial decrease in fit. In the fourth and final model we tested whether the factor loadings, factor correlations, factor variances and error variances are invariant across countries. Our results indicated that the data fit poorly with the a prori hypothesized model: $\Delta x^{2}(1124)=5703.43$ (p<0.01); GFI=0.72; TLI=0.94; $C F I=0.92$; $R M S E A=0.098$. The increase in chi-square between the two models is highly significant: $\Delta x^{2}(140)=4015.86$ ( $\left.p<0.001\right)$ and the remaining goodness-of-fit indices (TLI, CFl and RMSEA) also show a substantial reduction in fit.

In conclusion, we find support for configural invariance and partial support for measurement invariance. Our findings suggest that the factor loadings, factor correlations and factor variance are invariant across countries. The error variances are not invariant across countries. However, the invariance of error variances is typically regarded as the least important constraint (Marsh 1994). 


\subsubsection{Multilevel Analysis}

Our antecedents of customer satisfaction include variables at two levels of aggregation: the individual and the country level. Service quality evaluations occur at an individual or personal level, whereas cultural characteristics occur at the country level. Such data are designated as multilevel data (Brylk \& Raudenbush 1992; Steenkamp et al. 1999). The levels are hierarchical, in that customers are nested within countries. How to investigate hierarchically ordered systems has been a concern for a number of disciplines for quite some time. Conventional statistical techniques (e.g. ordinary regression analysis) ignore this hierarchy and, therefore, may lead to incorrect results (Bryk \& Raudenbush 1992; Hox 1995; Hox \& Kreft 1994). On the contrary. hierarchical linear models, also called multilevel models, are an effective approach to deal with hierarchically nested data structures (Hofmann 1997; Raudenbush 1993). Furthermore, a multilevel modell allaws for the estimation of cross-level effects (i.e. the interactive effects of individual and country level variables). This is possible, because the coefficients of the individuall level effects may be specified as random, partially explained by country level variables. Hence, this enables researchers testing hypotheses on how variables assessed at the country level affect relationships occurring at the individual level. For more details about multilevel analyses, we refer to appendix 1.

\subsubsection{Estimation}

For the conduction of the multilevel analyses the computer program MLwiN 1.0 (Rasbash et al. 1999) was employed which computes iterative generalized least squares (IGLS) estimates by means of an iterative approach known as the EM algorithm (Bryk \& Raudenbush 1992; Dempster et al. 1977; Goldstein 1995).

\subsubsection{Model Building}

In the present study, two level models were specified. First of all, level two contains eleven countries. Secondly, there are 7740 customers at level one. Consequently, the following strategy for model building was used. First of all, an intercept-only model (model A) has been estimated: a fully unconditional model (i.e. a model without predictors at any level), which decomposes the variance of the intercept into two independent random components, namely $\sigma_{e 0}{ }^{2}$ at the individual level and $\sigma_{u s}{ }^{2}$ at the country level. This model represents the (unexplained) variation of the outcome variable (i.e. overall customer satisfaction) at each level (individual and country). The 
second model (model B) is an extension: the variables under investigation are included at the individual level as well as at the country level to investigate how much of the total variance in the outcome variable can be explained by these added variables. The third model (model c) is extended with cross-level interactions between the individual level and country level variables to determine the contribution of these cross-level interactions to the model.

Table 2-1: Results of Multilevel Analyses

\begin{tabular}{|c|c|c|c|}
\hline & Model A & Model B & Model C \\
\hline Nnercepl (\%, & 6.5817 & $\begin{array}{l}\text { Fixed Part } \\
5.5180\end{array}$ & 5.8119 \\
\hline
\end{tabular}

Individual-lewel Coefficients":

Electronic Service $(\gamma / n)$

Service Call $(\gamma / 30)$

Service Wisil (m)

$\begin{array}{ll}.284(.0147)^{*} & .4679(.1960)^{*} \\ .3374(.0146)^{*} & .7699(.1842)^{*} \\ .4220(.0173)^{*} & .3919(.2187)^{*}\end{array}$

Country-level Coefficients ${ }^{\text {th }}$ :

Power Bistance (w)

Individualism (yoz)

Masculinity $\left(\gamma_{W}\right)$

Uncertainty A voidance $\left(\gamma_{04}\right)$

$\begin{array}{ll}-.0130(.0055)^{*} & -.0114(.0054)^{*} \\ .0115(.0061)^{*} & -.0081(.0061) \\ .0006(.0022) & .0009(.0022) \\ .0132(.0048)^{*} & .0101(.0047)^{*}\end{array}$

Cross-level Interactionss:

Power Distance $\times$ Electronic Service $\left(\gamma_{1}\right)$

Individualism $\times$ Electronic Service $\left(\gamma_{1}\right)$

Masculinity $\times$ Electronic Service $\left(\gamma_{13}\right)$

Uncertainty Avoidance $x$ Electronic Service $\left(y_{14}\right)$

Power Distance $x$ Service Call $\left(\gamma_{21}\right)$

Individualism $\times$ Service Call $\left(\gamma_{22}\right)$

Masculiaily $\times$ Serwice Call ( $\left.\gamma_{23}\right)$

Uncertainty A voidance $\times$ Service Call $\left(\gamma_{24}\right)$

Power Distance $\times$ Service V wisil $\left(\gamma_{1}\right)$

Indlvidualism $x$ Service Visin $\left(\gamma_{32}\right)$

Masculinity $\times$ Service Visit $\left(\gamma_{n}\right)$

Uncertanty Avoidance $\times$ Service Wisit $\left(y_{3}\right)$

$$
\begin{aligned}
& .0043(.0024)^{*} \\
& .0054(.0025)^{*} \\
& .0030(.0011)^{*} \\
& -.0044(.0022)^{*} \\
& .0024(.0023) \\
& .0051(.0023)^{*} \\
& .0013(.0010) \\
& -.0035(.0021)^{*} \\
& -.0002(.0026) \\
& -.0006(.0027) \\
& .0001(.001) \\
& -.0002(.0024)
\end{aligned}
$$

$\sigma_{*}{ }^{2}$ (individuat wer war)

$\sigma_{s i}^{2}$ (country-level var.
$2.6894(.0433)$
$.0367(0185)$

Random Part ":

$\begin{array}{rr}1.5275(.0246) & 1.5222(.0245) \\ .0067(.0118) & .0052(.0106)\end{array}$

Deviance

A Devianilue

$\Delta \mathrm{df}$
20645.23

\section{Model Fit: \\ 25268.73 \\ $4376.50^{\text {** }}$}

10
25240.28

$28.45 *$

12

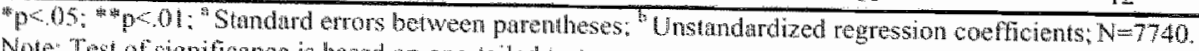

Note: Trest of significance is based on onemiled test. 


\section{6 .5 Results}

Table 2-1 presents the results of our multilevel analyses. The findings of madel $A$ indicate substantial variance at both levels, which implies that a multilevel approach is appropriate. The model fit, the predictive power of the different models, can be compared by a likelihood ratio test (Bryk \& Raudenbush 1992). Deviance is computed for each model and the difference between the deviance statistics ( $\Delta$ Deviance) has a $x^{2}$-distribution under the $H_{0}$ that the extended model (model B) does not predict significantly better than the reduced model (model A). Critical values of the $x^{2}$-statistic mean that the reduced model is too simple a description of the data (Kleinbaum et al. 1988). Furthermore, model $B$ reveals a highly significant $\Delta$ Deviance reflected in the sharp decreases in residual variances of the outcome variable at both levels, which implies that inclusion of the specified variables leads to a significant improved model. Regarding model $C$, though decreases in estimated residual variances are slight, the significant $\Delta$ Deviance reveals that this model still is a better specification than model $B$. Hence, adding the hypothesized cross-level interactions leads to a better predictive power of the model.

\subsubsection{Hypothesis Testing}

The results of model $C$ show a significant positive main effect for all three individual level perceived service quallity wariables: electronic service $\left(\psi_{10}=0.47\right)$, service call $\left(\gamma_{20}=0.77\right)$, and service visit $\left(\gamma_{30}=0.39\right)$ on overall satisfaction. Hence, we fail to reject lhypothesis 1: for the traditional (service visit) and technology-based (service call and electronic service) after-sales service delivery modes higher levels of perceived service quality result in higher overall customer satisfaction. Hypothesis 2 can also be accepted as indicated by the four non-significant cross-level interactions for service wisit. Therefore, the positive effect of face-to-face service visit quality on overall customer satisfaction is not significantly moderated by any of the four national cultural dimensions. With respect to the innovative service delivery modes hypothesis $3_{\mathrm{a}}$ is rejected: the cross-level interaction between power distance and perceived service call quality is not significant, indicating that for this service the positive relationship between service quality and overall customer satisfaction is universal across the participating countries. In support of hypothesis $3_{\text {bo }}$ however, the positive relationship between perceived bit-to-bit electronic service quality and overall customer satisfaction is significantly stronger in countries characterized by higher national power distance $\left(\gamma_{11}=0.0043\right)$. Next, national individualism significantly moderates the service quality-customer satisfaction relationship for both innowative service delivery 
modes. For service call $\left(\gamma_{22}=0.0051\right)$ as well as electronic service $\left(\gamma_{12}=0.005 .4\right)$ perceived service quality has a stronger positive impact on overall customer satisfaction in more individualistic cultures. Hypotheses, $4 a$ and $4_{b}$, therefore, are falled to be rejected. Masculinity, on the other hand, significantly moderates the positive service qualitycustomer satisfaction relationship only for the electronic after-sales service $\left(\gamma_{13}=0.0030\right)$, not for service call. Consequently, hypothesis $5_{3}$ is rejected and hypotheses $5_{t}$ is failed to be rejected. Finally, both for service call $\left(\gamma_{24}=-0.0035\right)$ and electronic after-sales service $\left(\gamma_{24}=-0.0044\right)$ a significant moderating effect is found for national cultural uncertainty. In support of hypotheses $6_{a}$ and $6_{6}$, the positive relationship between perceived service quality and overall customer satisfaction will be weaker in countries characterized by higher levels of uncertainty avoidance.

\subsection{Conclusion}

\subsubsection{Discussion}

In this chapter, we examined the moderating role of cultural dimensions in the customer evaluation formation of three after-sales service delivery modes. Our results seem to confirm that technology indeed is not independent of culture (Trillo 1997) and warn against overemphasizing similarities between cultures in the provision of services (Robinson 1988). This is in line with recent work in communications research which concludes that people tend to differ in their perceptions of technology-based forms of communication (Carlson \& Zmud 1999) and that contextual factors may influence the evaluation of mediated communication (Grudin 1994; Jones \& Marsh 1997). Therefore, our findings emphasize that it is important to take cross-cultural variation into account in the provision of international service intelligence.

Furthermore, our results enable to form an understanding of how national cultural characteristics play a role in the evaluation of innovative after-sales services delivery modes which are increasingly used by service providers nowadays. Concerning the cross-cultural universality of the service quality-customer satisfaction relationship, significant differences were found. For the traditional service delivery mode, the service visit, no moderating effects of cultural dimensions were found. Across all eleven countries, perceived service quality seems to be a key determinant of overall customer satisfaction with the manufacturer's after-sales service. We feel that this finding may be based on the following explanations. First, due to lower active participation in the service delivery process, a customep's cultural background and 
subjective cultural values are less likely to play a role. Overall satisfaction with the manufacturer's after-sales service is then mainly determined by the service engineer's performance and culture will not have an effect on this relationship. Related to this a second explanation can be sought in a phenomenon we refer to as cultural adaptation. In the day-to-day practice of traditional service delivery, face-to-face encounters are contextually rich enough to adapt to contextual and social cues. Furthermore, this type of service delivery commonly occurs within the same cultural context. Therefore, it is likely that cultural service adaptation automatically occurs and national culture is less likely to become an influential factor.

For innovative service delivery modes, characterized by higher levels of technology infusion and customer participation, customers" cultural background does seem to matter. In case of the voice-to-voice, call-center based services, both national individualism and uncertainty avoidance have a significant moderating effect on the service quality-satisfaction relationship. Customers in individualistic cultures seem to be more innovative and less bothered by the physical absence of the service provider. Quality of a voice-to-voice service seems to be valued more in such cultures and this reinforces the formation of overall customer satisfaction. For high uncertainty avoidance cultures however, a negative moderating effect was found. The inherent relatively higher perceived risk involved in telephone-based service delivery, diminishes the positive relationship between perceived voice-to-voice service quality and customer satisfaction in these cultures characterized by uncertainty avoidance. On the other hand, no significant moderating effects were found for power distance and masculinity. In a voice-to-voice service delivery mode there still is personal interaction between customer and service provider. For such interactions it is common to follow an 'act (i.e. speak) local' strategy, in which a customer is automatically being transferred to a contact employee with a similar cultural background or who is familiar with and adapts to cultural characteristics of the customer. Moreover, customers may be more used to telephone support in the case of after-sales service delivery. Finally, customers might not be aware of the technology (such as, for instance, computertelephone integration or CTI) involved. This could lead to the perception that this service delivery format is less distant and/or innovative and result in lesser impact of the power distance and masculinity dimensions.

The most pronounced consequences of cultural background are for electronic service delivery; the positive relationship between perceived service quality and overall customer satisfaction is moderated by a customer's cultural background, as a significant moderating effect was found for all four cultural dimensions. In cultures characterized by power distance, individualism, and masculinity the positive relationship between perceived service quality and customer satisfaction was 
significantly stronger. The distance created by technology seems in accordance with the distance in power structures and as people in individualistic cultures tend to be more innovative and trusting in exchange relationships with external parties this contributes to the formation of a customer's judgment regarding this new type of after-sales service delivery. In addition, customers in more masculine cultures will also value this service delivery mode more. They are more material- and achievementoriented and, therefore, will make more easily use of a new, technology-infused service and value adequate service quality more. Uncertainty avoidance, on the other hand, has the opposite effect. The positive relationship between service quality and overall custorner satisfaction was found to be significantly weaker in high uncertainty avoidance cultures. In these cultures, adequate quality of a high technology bit-to-bit service seems not to be valued additionally, most likely due to the higher level of uncertainty and perceived risk involved.

\subsubsection{Theoretical Implications}

Since the present study is a preliminary attempt to study the simultaneous effects of perceived service performance and national culture on customer service satisfaction several theoretical implications need to be taken into consideration. In this way, potential issues that merit future research efforts may be identified. First, a number of paradoxes seem to accompany technology (Mick \& Fournier 1998). For instance, technology could make customers feel competent and in control when it leads to a desired result, yet it could also result in a feeling of incompetence and loss of control in case something goes wrong. Due to the active role of the customer in technologyinfused service delivery modes there will unavoidably exist additional individual level contingency variables that might have an impact on customer service (dis) satisfaction. Future research, therefore, should incorporate more individual level variables, such as perceived control over the service process.

Second, at the aggregate (country) level additional variables should be taken into consideration. It has been suggested that the level of economic development in at country may play a role (Steenkamp et all. 1999). Moreover, the degree of Internet penetration and acceptance is likely to affect customer perceptions of new service delivery modes. It can be expected that the better a country is developed economically as well as technologically, the higher the acceptance of innovative, technology-infused services will be and the stronger the effect of perceived service quality on overall customer satisfaction will be.

Third, as illustrated by Hiltz and Johnson (1989), future work on technology-based applications should not assume that subjective satisfaction alone is sufficient 
indication of successful system implementation. Actual usage levels of an innovative service or its benefits, such as the impact on customer productivity, could be included in future research on the effectiveness of innovative service delivery modes.

A fourth limitation pertains to the updated Hofstede scores used to operationalize the cultural characteristics. These scores relate to work-related values, which according to Lynn and Gelb (1996) potentially do not equal the values people hold as consumers. Therefore, additional conceptualizations of national culture could be explored, such as Schwartz"s value scheme (Smith \& Schwartz 1997).

\subsubsection{Managerial Implications}

Our findings enable companies to nuance information on service channel performance. In an era of growing service globalization through the implementation of technology our results can be used for the formulation of more effective after-sales service management pollicies. Increased customer participation in innovative, technology-infused service delivery modes seems to create a need for adapting the manner in which these services should be provided. The main lesson to be learned from the present study is that, in comparison to more traditional service delivery modes (face-to-face), national cultural characteristics seem to be most influential in innovative after-sales services. In specific, for the voice-to-voice call-center service the impact of culture was moderate, whereas for the electronic service the positive relationship between perceived service quality and overall customer satisfaction was most strongly moderated by national culture. Service firms planning to centralize their service delivery modes (e.g., by means of installing pan-European contact centers), therefore, should acknowledge the potential effectiveness and perhaps even necessity of an adequate service adaptation approach. in correspondence with Johansson's (1997) recommendation that for the adaptation of services attention should be paid to aspects pertaining to the local environment cultural characteristics will partly determine the design of technology-infused service delivery processes.

More specifically, through user-centered designs in which culturally determined cognitions, values, and attitudes of users are stressed multicultural user bases could be served and cultural distances be overcome. Intelligent agents, designed to act like humans for collecting information, purchasing services or handling e-mail, need to 'understand' the cultural background of its users in order to be used effectively. As an example, in high uncertainty avoidance cultures an effective technology-infused service design would need to be different from that in low uncertainty avoidance cultures. The uncertainty likely to be associated with these services could be reduced, for example by providing guarantees, priority responses, service evidence in the form 
of progress reports, and the opportunity to establish personal contact with the service provider whenever required (Bitner et al. 2000). In addition, customers in low power distance and collectivistic cultures also would have to be approached differently than high power distance; individualistic customers. Our results seem to suggest that in such cultures, in which people tend to be less innovative, more risk-averse, and value personal contact, an emphasis on personalized service delivery could be more a effective strategy than a strong a focus on using technology for delivering the service. This does not imply that a technology-infused service delivery mode will be ineffective. Implementing an option to contact a service representative 'online' could, for example, already be sufficient. By carefully planning a multichannel service strategy in which the various service delivery modes play a complementary role, a service firm will be able to take culture's consequences for intermational service intelligence into account. 


\title{
Chapter 3
}

\section{The Impact of Incomplete Information on the Use of International Service Intelligence ${ }^{1}$}

\begin{abstract}
Whereas information on cultural differences has proven to be relevant, other types of information may be important as well. Given the substantial investments commonly associated with internationalization of services, it seems crucial to take information on financial consequences of senice investments into consideration. In this chapter, we examine the relative impact of the presence or absence of both cross-cultural and financial accountability information on user evaluative judgments and information utilization intentions. The results of an experimental study suggest that decisionmakers in international service firms are unlikely to ignore missing information Instead, they tend to adopt a strategy of inferential, assumption-based reasaning. Furthermore, the absence of information relevant to decision making in international services marketing has distinct evaluative consequences in terms of satisfaction, trust, and intention to use information.
\end{abstract}

\footnotetext{
${ }^{2}$ This chapter is largely based on: Birgelen, M.J.H. van, J.C. de Ruyter \& M.G.M. Wetzels (2000), "The Impact of Incomplete Information on the Use of Marketing Research Intelligence in international Service Settings: An Experimental Study", Journal of Service Research, 2 (4), 372-387.
} 


\section{The Impact of Incomplete Information on the Use of International Service Intelligence}

\subsection{Introduction}

During the past decade the services sector has been subjected to considerable growth. both on national as well as international levels. It has been identified as the fastest growing area of international trade (Bradley 1995; Terpstra \& Sarathy 1993). Globalization has become an effective competitive strategy for service firms due to a combination of structural, market, and technological changes (Segal-Horn 1994). stagnant domestic markets and growth opportunities across geographical borders cause service firms to broaden their business horizons (Bradley 1999).

It is generally agreed upon that services can be characterized by higher perceived purchase risk in comparison to goods (Bateson 1995). An important reason for this pertains to the fact that services are hard to standardize (Guseman 1981). Other reasons are limited information availability for consumers due to higher levels of experience and credence attributes of services (Parasuraman et al. 1985) and involvement of customers in the production process of services. (Bateson 1995). In comparison to national services, the associated purchase risk is likely to be even higher for foreign services, due to difficulties with standardizing services internationally and higher unfamiliarity with the service or its provider. In total, this will result in comparatively high levels of international uncertainty for service firms.

Furthermore, when engaging in international activities, service organizations are facing the challenges and barriers associated with internationalization strategies (Cowell 1993). They come into contact with new business environments as well as unfamiliar cultures. Since culture is an integral element of the service encounter (Bradley 1999), inevitably this will result in higher degrees of uncertainty. Cultural customs and practices are highly determinant for customers service expectations, perceptions, and ultimately evaluations. A service firm's ability to effectively deal with culture-based differences in expectations, perceptions, and evaluations, as well as in forelgn customs and practices will determine its international success or failure.

In dealing with international uncertainty, serwice firms are continuously in need of marketing intelligence. For the development of successful international marketing strategies, detailed market intelligence is a prerequisite (Craig and Douglas 2000). Among the most common ways of obtaining such information is conducting international marketing research. According to Newman (1962), marketing research 
can supplement the intuition and assumptions of the marketing decision-maker. However, "one of the unfailing realities of marketing problems is that information about the situation is often incomplete and of questionable accuracy" (Nylen 1990, p. 8). Consequently, in addition to uncertainty inherent to international environments, decision-makers in service firms will be confronted with informational uncertainty as well.

The purpose of the present study is to gain more insight into the issue of incomplete intelligence and its consequences for decision-making in international service settings. We aim at investigating what the impact will be of incomplete international service intelligence (1) on the way in which decision-makers in international service organizations handle this lack in information, (2) how they will evaluate the intelligence that is available, and (3) whether they will use it for decisionmaking purposes. Insight in the consequences of incomplete information enables providers of international service intelligence, such as marketing research agencies or internal research departments, to better understand the underlying processes and the effects of informational uncertainty. Information management policies can then be developed that ultimately help decision-makers in service organizations to more effectively deal with uncertainty.

The chapter is structured as follows. First, we will provide a brief synthesils of the extant literature on decision-making uncertainty and missing information and more explicitly discuss their role in (services) marketing decision-making. Next, we will address two distinct informational topics of decision-making relevance for international service settings: (1) information on the cross-national comparability of research data and (2) information on the financial accountability of international service investments. The first type of information aims at reducing environmental, culture-based, uncertainty, the latter serves to decrease strategic uncertainty of international service activities. 5ubsequently, we will formulate our research hypotheses. After having reported on an experimental study designed to test our hypotheses, we conclude the chapter with a discussion of the results and present a number of theoretical and managerial implications.

\subsection{Uncertainty, Missing Information, and Marketing Decision-Making}

Uncertainty is a well-studied phenomenon in academic decision-making literature. This originates from the fact that it is quite influential on effective decision-making practices Kahneman et al. 1982; McCaskey 1986; Orasanu \& Connolly 1993; 
Thompson 1967). When reviewing the stream of literature on uncertainty, it soon becomes clear that there is no unambiguous definition of this concept available. Lipshitz and Strauss (1997) integrate existing definitions and provide a quite comprehensive conceptualization of uncertainty. These authors identify three types of uncertainty, namely (1) incomplete information, (2) inadequate understanding of information due to conflicting meanings, and (3) undifferentiated informational alternatives that are equally positive or negative.

Decision-makers have been found to cope differently with these distinct types of uncertainty (Lipshitz \& Strauss 1997). One of the mostly used ways of coping with incomplete information is so-called assumption-based reasoning, i.e. filling gaps in knowledge by making assumptions through extrapolation on the basis of available information (Cohen 1989). Reducing uncertainty, for example by collecting adlitional information, is used mostly to deal with an inadequate understanding of information. Finally, undifferentiated alternatives are handled by weighing the pros and cons of each alternative. An additional way of dealing with uncertainty is by suppressing or denying it. This particular strategy was found to be used the least by decision-makers.

In the present study, the focus is on the impact of uncertainty caused by having incomplete information in international service settings. Despite the importance of complete information for reducing international uncertainty, the consequences of information incompleteness represent an underdeveloped area in the intermational services marketing literature. To date, research on the effects of missing information on decision-making has featured most prominently in organizational behavior sciences, human decision processes and applied psychology literature (e.g., Jagacinski 1991; Slovic \& MacPhillamy 1974; Yamagishi \& Hill 1981; Yates et al. 1978).

In these areas, research mainly focuses on how decision-makers form evaluative judgments about alternatives that vary in the amount of information, by looking at how these decision-makers combine and integrate existing information and how they deal with missing information. For instance, in the context of personnel decisions, lagacinski (1991) demonstrated that decision-makers did not ignore important missing information about job candidates but predicted the missing information from available information, then make an evaluation about the candidate and penalize the candidates for whom this information was missing.

In marketing-related areas, the issue of missing information has been studied especially in relation to consumer behavior toward goods. Several studies investigated how consumers handle missing information when having to form evaluations about products and making purchase decisions. Most of these studies support the formal inference processing model, finding its essence in the earlier-mentioned principle of assumption-based reasoning (Ford \& Smith 1987; Gardial \& Biehal 1987; Johnson 
1989; Johnson \& Levin 1985\%. The model predids that consumers make inferences on a product's unstated attributes on the basis of stated attributes that serve as informative cues. The presented and inferred attributes are then integrated to form an overall evaluation, preference, or choice (Lim \& Kim 1992).

With respect to this evaluation, Johnson and Levin (1985) conclude that the greater the amount of missing information, the less favorable a product's evaluation. As compensation for the missing information consumers might try to collect additional information. However, "...the ability to form accurate inferences, even with considerable mental effort, can reduce the total effort associated with the choice process by eliminating or significantly reducing the search for the missing information" (Ross \& Creyer 1992, p. 15). This illustrates that in many marketing decision-making situations, inference formation is an essential strategy in the process of forming evaluations and that it represents an interesting field for further academic investigation.

The question whether findings from the various research domains, including the product literature, could be extended to the intermational services setting has been left unanswered. Incomplete information will function as an "uncertainty multiplier effect", implying that it will increase the uncertainty that is inherent to international services. Before examining the effects of incomplete information in international service settings, however, the question should be raised of what matters are important for adequate decision-making in international service management. In the next section, two such informational topics will be introduced on the basis of which our research hypotheses will be formulated.

\subsection{Informational Topics for Decision-Making in International Service Settings}

Different types of information tend to differ in degree of decision-making relevance across business settings. In this chapter, the focus will be on two informational topics that are likely to be of relevance for decision-makers in an international service setting: information on cross-national comparability of research data and information on financial accountability of international service investments. 


\subsubsection{Information on Cross-national Comparability of Research Data}

The impact of a country's culture on the international marketing of services is quite substantiall. For services, cultural differentiation will be one of the most important causes of international uncertainty. As Dahringer (1991, p. 7) states "barriers to the international marketing of services are due largely to the close cultural relationships between a society and the services offered in that society". Due to the high levels of heterogeneity and subjectivity, services are hard to standardize (Rust et al. 1996). This holds for national services, but even more for international service activities. A country"s culture is an integral element of many services (Bradley 1999) and cultural factors form a major obstacle to standardization (Buzzell 1968).

Services are often strongly culture bound and their demand is influenced by a country's society (Bradley 1999). Therefore, variations in service quality performance perceptions can be partially culturally determined. Service issues important in one society, for example the way in which a service is provided, may be found less relevant in another society in which it might be the outcome of the service process that counts. In addition, organizations crossing national borders will be confronted with "differences in economic wealth and levels of literacy, coupled with linguistic heterogeneity and cultural diversity in marketing environments throughout the world" (Douglas \& Craig 1983, p. 11). Of the entire service delivery process, the service encounter is the stage in which a society's culture is likely to be most influential. It is at this stage when the interaction between the international service organization and its foreign customers takes place and the organization is directly confronted with local customer preferences and practices.

Higher levels of unfamiliarity with foreign markets combined with the cultural diversity across countries create a need for reliable marketing research information. However, due to the same cultural differentiation creating this need, difficulties will arise when comparing research data cross-nationally. Information often might not be readily comparable across countries. Important service concepts may be understood differently across countries or participants from whom the information is being obtained might react differently to measures used for data collection. Theoretically, these problems form serious threats to both construct and measure equivalence, respectively referring to cross-cultural equivalence in constructs to be studied and equivalence in the operational measures in cross-cultural settings (Craig \& Douglas 2000). In ordep for international marketing research data to be comparable across countries, equivalence both in content as well as methodology is of crucial importance (Dawar \& Parker 1994; Craig \& Douglas 2000). Equivalent and standardized measures 
should be developed, athough these may entail some loss of precision and accuracy in any given culture and country (Elder 1976; Przeworski \& Teune 1970).

Information that is comparable cross-nationally reduces the environmental uncertainty and can be used validly and reliably for the purpose of decision-making in international service settings. For establishing equivalence, marketing researchers must first carefully design appropriate research methods. Furthermore, analysis techniques must be applied to formally check the lewel of equivalence in multicountry data (e.g. Mullen 1995; Styles 1998). Decision-makers are likely to feel more comfortable with their decisions when being convinced that thorough attention has been paid to the international validity of research information. Therefore, providers of international marketing research information should pay explicit attention to the level to which crossmational equivalence in the data has been established and checked when providing intelligence to decision-makers in international service organizations.

\subsubsection{Information on Financial Accountability of International Service Investments}

The second informational topic relates to the financial accountability of international service investments. Investments in service activities and service quality are usually made with the objective of improving performance and customer satisfaction. Investing in customer satisfaction is important to business organizations since "...customer satisfaction drives future profitability" (Anderson \& Formell 1994, p. 13). In the goods industry, the relationship between marketing investments and business performance has been studied extensively. One of the most well-known examples is the PIMS (Profit Impact of Marketing Strategies) program, sponsored by the Strategic Planning Institute (SP) (cf. Buzzell \& Gale 1987). Some of the most important. conclusions were that market share is positively related to Return on Investment (ROI) and that product quality is an important determinant of market share. Other studies reported a direct positive association between product quality and ROI (Buzzell et al. 1975: Schoeffler et al. 1974).

Also in the services industry the necessity of gaining insights into the financial consequences of investments has been acknowledged. According to Rust et al. (1995. p. 59) "How to make profitable decisions about quality expenditures is the key managerial problem". Service quality improvement efforts need to be financially justified or accounted for. For this purpose managers need a method that helps them to direct their limited resources to those areas from which they get the greatest response. The "Return on Quality" (ROQ) approach, developed by Rust et al. (1995), is such method in which the change in the net present value caused by the quality improvement is related to the necessary extra expenditures. It allows managers to 
compare alternative quality improvements on the basis of their $R O Q$ and has been proven useful in several service industries, such as hotel and banking services.

When conducting a marketing research project on the service quality of an international service firm and its customers' satisfaction level, the outcome is likely to consist of conclusions and managerial recommendations aimed at improving service quality where necessary. Due to the ever-growing urge to account for financial investments, information on the financial implications of service expenditures will be highly appreciated by decision-makers. The relevance of this particular type of information is illustrated by Lipe's (1998) finding that some market measures, such as variance of returns, had an actual effect on investment decisions and risk assessment. Information on investment consequences provides an opportunity to reduce the operational uncertainty associated with international service investment decisions. Therefore, information on the accountability of international service investments represents an useful issue in a marketing research project. After having discussed two important informational topics, we now will continue with the formulation of our research hypotheses concerning information incompleteness with respect to these topics and its consequences for decision-making in international service settings.

\subsection{Developing Hypotheses on Incomplete Information in International Service Settings}

Service firms seeking information on the international markets they enter or are active in often hire marketing research agencies to conduct marketing research projects for them. Deshpande and Zaltman (1982, p. 15) state that "reliance on external research agencies is a common if not the most common approach to conducting research". Mostly, these projects will result in research reports presenting concrete results and managerial recommendations for the international service organization. On the basis of the previous discussion, explicit attention could be paid in such research reports to the level of cross-national comparability of research data. This communicates to decision-makers that techniques have been used to make the information comparable across countries and that it can be readily used for operational decision-making. On the other hand, it is also possible that nothing is being mentioned on the comparability of the data, consequently leading to incomplete information on this issue.

Similarly, reports may differ in the level to which attention is being paid to the financial accountability of international investments in service quality. Our point of 
departure in this experimental study is that incomplete information on both topics. will result in higher levels of decision-making uncertainty in international service settings. To set the stage for further analyses our first hypothesis focuses on how decisionmakers in an international service firm will deal with incomplete information. Subsequently, several hypotheses focus on the evaluative effects of varying levels of information presence on satisfaction with, trust in, and finally intention to use the information avallable in an intermational research report. Figure 3-1 presents our conceptual framework with respect to the experimental factors and the evaluative dependent variables.

\section{Figure 3-1: Conceptual Framework}

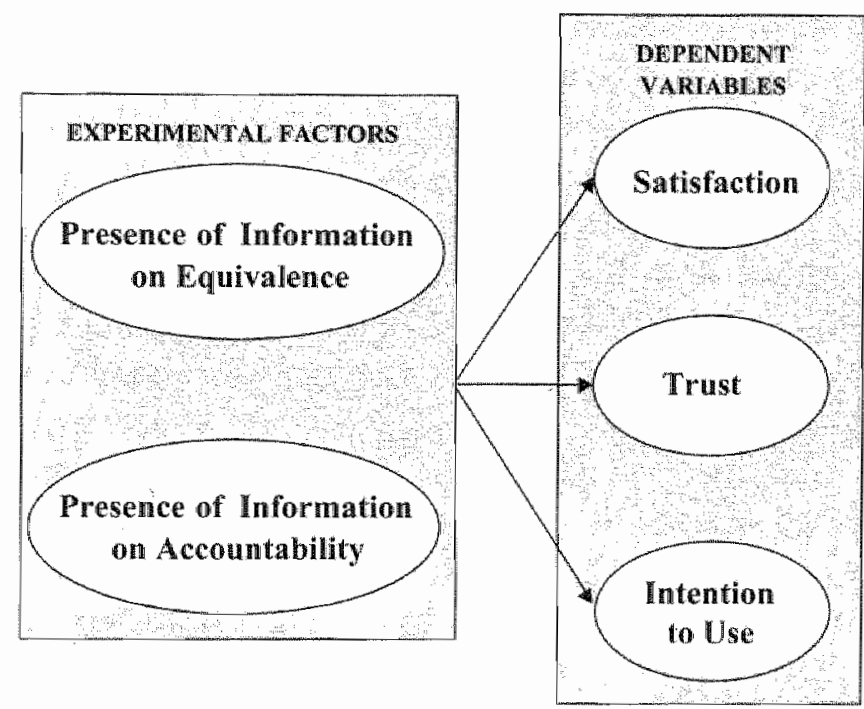

As mentioned before, decision-makers can deal with uncertainty in several ways. In the case of uncertainty caused by missing information, it was found that missing information is unlikely to be ignored or denied by decision-makers (Lipshitz \& Strauss 1997). Potter and Beach (1994) concluded from an experimental study that specific missing information on attributes is regarded as an important violation when having to make decisions. This violation was found to strongly influence the screening process of alternatives between which a choice had to be made, resulting in favorable evaluations of more complete alternatives.

Other studies have shown that decision-makers will tend towards assumptionbased reasoning and extrapolate or predict the missing information from other avallable information (Ford \& Smith 1987; Gardial \& Biehal 1987; Jagacinski 1991; 
Lipshitz \& Strauss 1997\%. The main adwantage of using assumption-based reasoning is that it enables experienced decision-makers to act quickly and efficiently on the basis of limited amounts of information (Lipshitz \& Ben Shaul 1997). For decision-making in international services, other available information might for example be the information present in the report, information obtained from other related studies, and knowledge based on a decision-maker's own experience. These informational sources can be used to make inferences about the missing information, which then are combined with the available information in order to evaluate the overall decisionmaking usefulness of the research report presented. Correspondingly we hypothesize that:

Hypothesis 1:

When being confronted with a marketing research report in which information would be missing decision-makers in international service settings are more likely to make predictions about the missing information on the basis of ather available information than to ignore it.

Hypothesis 1 relates to how decision-makers objectively deall with incomplete international marketing research information. When studying the use or non-use of such information, a focus on subjective evaluative judgments is useful as well. Since conducting a marketing research project can be considered a service provided by the research agency to a client, we adopt a perspective in which customer evaluative judgments are central (e.g., Parasuraman et al. 1985, 1988). Several evaluative criteria in the context of services marketing have been developed in the literature, including customer satisfaction and trust between partners.

Satisfaction can be described as a customer's cognitive and affective evaluation of a product or service being delvered to him/her by a specific provider (olver 1997). Satisfaction was fiound to be influenced by the level of penceived service quality (Cronin \& Taylor 1992; Oliver 1993; Parasuraman et al. 1994; Rust \& Oliver 1994). In a study on relationships between providers and users of marketing research intelligence, Moorman et al. (1992, p. 315) define trust as "a willingness to rely on an exchange partiner in whom one has confidence". We adapt this definition and define trust in research information as "a willingness to rely on marketing research information in which one has confidence ${ }^{\text {nv }}$. Moorman et al. (1993) found that, among other factors, trust was positively influenced by a researcher's willingness to reduce research uncertainty. This could be achieved by providing information that is as complete as possible. 
Menon and Varadarajan (1992) suggest that a receiver's perception of information quality influences the degree to which the information is acted upon. Deshpande and Zaltman $(1982,1984)$ found a positive relationship between quality of the contents of a research project and the extent of use of research information. In managerial decision-making situations, alternatives in which information was missing were found to be rejected more readily (Potter \& Beach 1994). Also in marketing decision-making it can be concluded from several studies that alternatives with missing information tend to be devalued by consumers (Huber \& McCann 1982; Johnson \& Levin 1985). integrating the above, we expect that complete marketing research information leads to more positive attitudinal (satisfaction and trust) and behavioral (intention to use) evaluative judgments compared to when information is missing in an international research report. Therefore, we formulate the following set of research hypotheses:

\section{Hypotheses 2:}

Decision-makers in international service settings having to make decisions on the basis of a marketing research report that contains complete information on both cross -national data equivalence and financial accountability of international service investments...

$\mathrm{H}_{2 \mathrm{a}}$ : are more satisfied with;

$\mathrm{H}_{2 \mathrm{~b}}$ : have higher levels of trust in;

$H_{x x}$ : and have a higher intention to use the avalable information in the report ... than decision-makers having to make decisions on the basis of a report that is missing information on both topics.

In practice, decision-makers are likely to value various types of information differentially. Information on the financial consequences of planned investments could be perceived as more meaningful and involving than research-methodological information on whether or not data have been corrected for cross-national differences. This is driwen by the earlier mentioned managerial need of knowing the financial consequences of (service) investments: "In an era of cost cutting, quality expenditures must be made financially accountable" (Rust et al. 1995, p. 59).

From the attribution literature (Gilbert et al. 1988; Newman \& Uleman 1989) it is known that if information creates more cognitive unrest, individuals will process this information more deeply and will develop attributions. Furthermore, accountable subjects, having to justify their judgments and decisions to others, will display more search efforts and examine more information (Lee et al. 1999), whereas lower issue involvement decreases information processing efforts Maheswaran \& Meyers-Levy 1990). In contrast to equivalence information, information on service investment accountability is highly relevant for a manager's perception of the environment. It will 
create higher levels of involvement, mainly because it directly confronts decisionmakers with the financial consequences of their decisions, and thus is likely to be processed more elaborately. Literature on distinctiveness supports this notion and indicates that relevant and distinct stimuli are more deeply processed (Sanbonmastu et al. 1991) and more easily remembered (McDaniel et al. 1995). We postulate that information on cross-national equivalence of research data is likely to be of lesser practical relevance for decision-makers in international service settings and, therefore, less influential on attitudinal and behavioral evaluative judgments in comparison to information on financial accountability of international service investments. Consequently, we hypothesize that:

\section{Hypotheses 3 :}

Decision-makers in international service settings having to make decisions on the basis of a marketing research report that cantains no information on cross-national data equivalence and complete information on financial accountability of international service investments...

$H_{\text {3a: }}$ are more satisfied with;

$H_{3 b}$ : have higher levels of trust in;

$\mathrm{H}_{36}$ : and have a higher intention to use the available information in the report ... than decision-makers having to make decisions on the basis of a report that contains complete information on equivalence and no information on accountability of investments.

The previous set of research hypotheses differentiates between the effects of incomplete information on the two informational topics, based on the notion that decision-makers are likely to make a cognitive distinction between relevant and less relevant information. However, it can also be expected that both types of information interact with each other. The magnitude of the presence effect of relevant accountability information might very well depend on the level of information avallable in the international research report on cross-national data equivalence. This originates from the thought that decision-makers will additionally value apparently less relevant information as soon as they feel at least being provided with information of higher perceived relevance. This corresponds with the so-called adding model, which focuses on how decision-makers combine information when making evaluations about alternatives (Jagacinski 1991). Adding models state that more information (e.g." on cross-national data equivalence) increases the total value of an alternative as long as this information is not negatively valued by the decision-maker. 
Therefore, with respect to the interaction between both types of information, we hypothesize that:

\section{Hypotheses 4:}

The effect of complete information on financial accountability of international senvice investments will be more positive in terms of ...

$H_{43}$ : satisfaction with:

$\mathrm{H}_{40}$ : trust in, and;

$\mathrm{H}_{4, \mathrm{c}}$ : intention to use the avallable information in the report

... for decision-makers in international service settings having to make decisions on the basis of a marketing research report that also contains complete information on cross. national data equivalence than for decision-makers having to make decisions on the basis of a report with no information on equivalence.

In the next section, we will report on the results of an experimental study designed to test our hypotheses.

\subsection{An Experimental Study}

\subsubsection{Experimental Design}

In order to test our hypotheses, we used a between-subjects, fixed-effects factorial design consisting of two factors. Information on cross-national data equivalence (EI) was manipulated on two levels: (1) providing complete information on data equivalence and (2) providing no information on data equivalence. Information on financial accountability of international service investments (AI) was also manipulated on two levels: (1) providing complete information on financial accountability and (2) providing no information on financial accountability. Consequently, we arrived at a full $2^{2}$ factorial design, allowing us to explicitly take interaction effects into account (Keppel 1991; Kirk 1982).

\subsubsection{Stimulus Materials}

As stimuli for evoking responses, we developed distinct scenarios to be shown to decision-makers working in an international service setting and who use international marketing research information on a regular basis. The purpose of these scenarios was 
to help decision-makers put themselves into the situation in order to test our hypotheses. Each scenario contained a description of a hypothetical international marketing decision-making situation. The scenarios were developed after in-depth interviews both with marketing researchers and decision-makers. This was done to ensure that scenarios depicted a realistic situation and were worded realistically.

Each scenario started with an introduction to clarify the setting of the decisionmaking situation. In this introduction, it was explicitly mentioned that respondents should picture themselves in a situation in which they were confronted with an imaginary international marketing research report provided by an external marketing research agency. Respondents had to be real-life decision-makers. They were selected from a list of employees working at the international headquarters of a large, Dutch office equipment manufacturing company. It was stated that, on the basis of the report, they would have to make decisions regarding (the improvement of) the manufacturer's international service quality policy or assist in the decision-making process regarding this policy.

The report was said to contain information about a (fictive) study on international customer satisfaction with various service aspects relevant for this particular type of industry. This study had been conducted internationally among customers of operating companies located in several countries. In each scenario it was mentioned that the report contained all the standard marketing research information, such as information on research design, data collection methods, sampling techniques, sample composition, and results and recommendations regarding international customer satisfaction with the manufacturer's service. The manipulation occurred with respect to the amount of information on cross-national data equivalence and financial accountability of international service investments said to be present in the research report.

The two levels for equivalence information were incorporated in a scenario by stating that decision-makers should imagine themselves in a situation in which they would like to compare customer satisfaction scores between the different international operating companies of the manufacturer. They were then notified of the problem that arises when comparing satisfaction scores across countries. This was done by pointing respondents at the fact that differences in familiarity with measures and scaling procedures may lead to incomparable scoring across populations. Furthermore, due to differences in culture, a score obtained in one country may not have the same meaning or conceptual interpretation in another country. Therefore, different procedures and conceptualizations of the issue under study may be effective in different cultures and countries. 
In the experimental design, one group of decision-makers (with complete information on cross-national data equivalence) responded to a scenario in which it was explicitly stated that the marketing research agency did everything possible to establish cross-national equivalence in the customer satisfaction data and that valid national comparisons could be made. Another group was confronted with a scenario in which it was explicitly stated that no information on the establishment of data equivalence was present in the research report and, therefore "that a valid comparison could not be guaranteed.

With respect to information on financial accountability of international service investments, decision-makers were explicitly notified of the importance of knowing what additional costs will be incurred by international service quality improvement efforts and what the actual benefits and pay-off will be. One group of decision-makers was told that the imaginary research report included an appendix presenting the results of various cost/benefit analyses. These analyses clarified what the estimated costs and benefits would be of service quality improvement efforts on the basis of recommendations given in the report. Another group would read a scenario, stating that no such information was present in the report. In total, four scenarios were composed based on the combination of manipulated amounts of information on both topics. For the purpose of illustration, a final sample scenario is included in appendix 11.

\subsubsection{Pretest}

Using simple random sampling, 15 actual decision-makers were selected for the pretest. The pretest had two functions: (1) to assess whether the desired state was induced by the manipulations of the independent experimental factors and (2) to assess the reliability of the dependent measures. The subjects participating in the pretest were interviewed immediately after exposure to the manipulation (Perdue \& summers 1986). The interviews revealed that the manipulations were successful in inducing the desired state of mind. Only minor adaptations to the four decision. making scenarios were necessary. Moreover, it became clear from preliminary analyses that the dependent measures showed sufficient reliability in terms of coefficient alpha.

\subsubsection{Procedure}

As mentioned participants in the experiment had to be real-life decision-makers. All the participating decision-makers, were located at the international headquarters of the manufacturing organization. These headquarters consist of staff departments 
that, by formulating company policies, centrally guide the operating companies in a great number of countries. To reach an acceptable level of external validity, an essential criterion was that respondents to be included in the final sample were regular users of internationall marketing research information who had experience in interpreting it. Potential respondents were randomly assigned to the four treatments.

Initially, 126 respondents participated in our study. However, by evaluating control questions assessing a respondent's job title (used for the determination of whether respondents were likely to be in a position in which they have to use information) and usage rate of international marketing research information (considered a proxy for interpretation experience), 6 respondents were excluded from our sample, this because they were not likely to have the intended usage and experience level. Consequently, the final sample consisted of 120 usable decision-makers equivalently distributed across the four random experimental groups. Among others, these decision-makers were active in business analytical positions (7\%; e.g., business analyst, service business analyst), strategic positions ( $9 \%$; e.g., strategic planner), productrelated positions (23\%; e.g., product manager, product specialist), and service-related positions (48\%; e.g., service manager, service marketer).

Since we expected relatively large effects (Cohen 1992; Sawyer \& Ball 1981; Stevens 1986) for all treatments and anticipated the dependent variables to be highly intercorrelated (Stevens 1986), a sample size of approximately 25 per cell would suffice to achieve a power (1- $\beta$ ) of 0.80 at an $\alpha=0.05$ (Cohen 1992; Sawyer \& Ball 1981). Each respondent received a booklet which, in addition to the control questions, included instructions, a description of the scenario and the satisfaction, trust, and intention to use measures. Additionally, measures were included for assessing a decision-maker's strategy of dealing with missing information. Furthermore, manipulation checks were added to assess whether the state intended by the two independent variables was induced (Perdue \& Summers 1986).

\subsection{Results}

\subsubsection{Manipulation Checks}

The results of the manipulation checks suggested that there were significant differences between the experimental levels as intended by the design. The cells with complete and no information on cross-national data equivalence differed significantly $\left(F_{i .115}=9.08[p=0.0032]\right)$ on the statement 'This study would enable me to create al valid 
sequence of the various operating companies on the basis of customer satisfaction scores: Cells with complete and no information on financial accountability of international service investments differed significantly $\left(F_{1,915}=6.74[p=0.0107]\right)$ on 'This study would provide me with adequate input for service imvestment decisions. Furthermore, Sternthal et al. (1987) argue that ulitimately the effectiveness of the manipulations is reflected in the pattern of the resulting data.

\subsubsection{Validity and Reliability of Dependent Measures}

We carried out principal component analyses to assess whether the items for the dependent variables would load on separate components. These analyses showed that each item loaded (>0.5) on only one component and that items grouped together as intended. This indicates discriminant vallidity and that the three measures are tapping different concepts. Satisfaction with the information in the imaginative research report was operationalized by 4 items on a 7-point Likert-type scale (e.g., "I would be satisfied with the contents of this research report', "I would be satisfied with the results of this research report", and "This research report would be positive for our organization'). These items were partly based on a measurement instrument developed by Oliver (1980). A composite measure for satisfaction, as well as for trust and intention to use the available information, was calculated by averaging the items. The coefficient alpha for satisfaction was 0.83 .

Trust in the information was operationalized by 3 items on a 7-point Likert-type scale which were adapted from a scale developed by Moorman et al. (1992) (e.g." "I would trust the contents of this research report", "I would trust the results of this research report', and 'I would be confident in taking decisions on the basis of this research report') (coefficient alpha $=0.81$ ). The items for measuring a decision-maker's intention to use the information originated from a scalle by Maltz and Kohli (1996). We conceptualize information use as the extent to which the deciston maker intends to use the information to understand his or her work environment and to make and implement service quality decisions, thereby respectively covering both conceptual and instrumental use of research information (Menon \& Varadarajan 1992). The instrument consisted of 8 items measured on a 7-point Likert-type scalle (e.g. "It is very likely that the information of this study would improve my understanding of how customers perceive our" service quality", "The information of this study would improve the effectiveness and efficiency of our service quality policies", and 'It is very likely that I would use the information of this study for improving our service quality policies") (coefficient alpha $=0.91$ ). 
Finally, the way in which decision-makers would (hypothetically) deal with missing information (predicting versus ignoring) was operationalized by 3 items on a likert type scale for each strategy. These items were presented to all respondents, also to the ones who were confronted with the complete information scenario. These respondents were asked to imagine themselves in a situation in which (some) information was missing. The items were developed specifically for the purpose of this study (predicting: e.g." 'On the basis of the information that is avallable in the research report 1 would make inferences about the missing information and use these inferences together with the available information to form a judgment about the report"; ignoring: e.g., "I would not be bothered by the fact that certain information is missing in the report and base my judgment solely on the information that is avallable in the report'). The instrument for 'predicting' had a coefficient alpha of 0.73 . for 'ignoring' this was 0.81 . Overall, therefore, all reliability scores wellmexceed the threshold value of 0.70 as proposed by Nunnally and Bernstein (1994).

\subsubsection{Testing Assumptions of MANOVA and Dependent Variables}

Given the relatively large sample size and the robustness of MANOVA to departure from multivariate normality (Stevens 1986; Tabachnik \& Fidell 1996), violations of multivariate normality are not expected to be severe. In addition, inspection of the histograms, normal-probability plots, skewness and kurtosis for each dependent measure for each cell showed only slight departures from normality. Another assumption underlying MANOVA is equality of variance-covariance matrices. This assumption can be tested using Box's $M$ test for homogeneity of dispersion matrices. However, this test is usually not very useful, as it is extremely sensitive to multivariate nonnormality (Stevens 1986: Tabachnik \& Fidell 1996).

Finally, power analysis revealed that the power level was well above 0.8 for the significant effects ( $\alpha=0.05$ ). If the dependent variables are uncorrelated, MANOVA is superfluous. In such a case, one might be able to rely on univariate ANOVAs (one for each dependent variable). The pooled within-groups correlation matrix revealed relatively high correlations between the dependent variables, thereby supporting the applicability of MANOVA.

\section{6 .4 Results of MANOVA}

Before testing our hypotheses, we provide the results of MANOVA, conducted to assess whether significant main and interaction effects of the independent factors on the dependent variables exist. Research into the robustness of the statistics available for 
MANOVA Suggested that the Pillai-Bartlett trace criterion [V] might be the most robust statistic for general protection against departures from multivariate nomally and homogeneity of variance-covariance matrices (Stevens 1986; Tabachnik \& Fidell 1996). Therefore, we will only report the Pillai-Bartlett trace criterion and its f-approximation. However, it must be noted that all four rival tests (Wilks" likelihood ratio criterion [W], Hotelling-Lawley trace criterion [T], Roy's largest root criterion [R], Pillai-Barlett criterion [V]) are asymptotically equivalent in large samples. The results of MANOVA are summarized in table $3-1$.

\section{Table 3-1: Results of MANOVA}

\begin{tabular}{|c|c|c|c|c|}
\hline Effect & Pilla-Bsartlett Trace [V] & $F^{a, d}$ & p-walue & $n^{2,6}$ \\
\hline \multicolumn{5}{|c|}{ Main Effects } \\
\hline $\begin{array}{l}\text { Equivalence } \\
\text { Information (EI) }\end{array}$ & 0.04 & 1.50 & $\mathrm{p}=0.219$ & 0.04 \\
\hline $\begin{array}{l}\text { Accomntability } \\
\text { Infomation (AI) }\end{array}$ & 0.13 & 5.68 & $p=0.001$ & 0.13 \\
\hline \multicolumn{5}{|c|}{ Two-Way Interaction } \\
\hline $\mathrm{EI} * \mathrm{AI}$ & 0.13 & 5.71 & $p=0.001$ & 0.13 \\
\hline 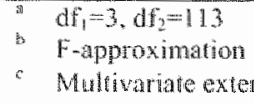 & nof $\eta^{2}$ & & & \\
\hline
\end{tabular}

As can be concluded from table 3-1, we found that the presence of information on financial accountability of international service investments has a significant main effect (on all three dependent variables), indicating that the means on the dependent variables are unequal for the two levels in which accountability information is present in the iriternational research report (Accountability information: $V=0.13 ; F_{3,13}=5.68$ $\left.[p=0.001] ; \eta^{2}=0.13\right)$. For the presence of information on cross-national data equivalence no significant main effect was found (Equivalence information: $V=0.04$; $F_{3,113}=1.50[p=0.219] ; m^{2}=0.04$ ).

Additionally, we found that the two way interaction between both types of information showed a significant effect $\left(V=0.13 ; F_{3.13}=5.71[p=0.001] ; \eta^{2}=0.13\right)$. As a consequence, the main effects in isolation will not yield a faithful picture of the results of the experiment (Keppel 1991; Kirk 1982). The cell means for the interaction between equivalence information and accountability information are presented in figure $3-2$ for all three dependent measures. The interaction effect turned out to be significant for both satisfaction with and trust in the available information in the international research report (Satisfaction: $F_{1,215}=6.00[p=0.016]$; Trust: $F_{3.115}=5.43 \quad[p=0.022]$ ). For intention to use the information the interaction effect was not significant (intention to use: $\left.F_{1,1,5}=0.20[p=0.656]\right)$. 
Figure 3-2: Graphical Representation of Means for Two-Way Interaction Between Presence of Equivalence and Accountabillity Information

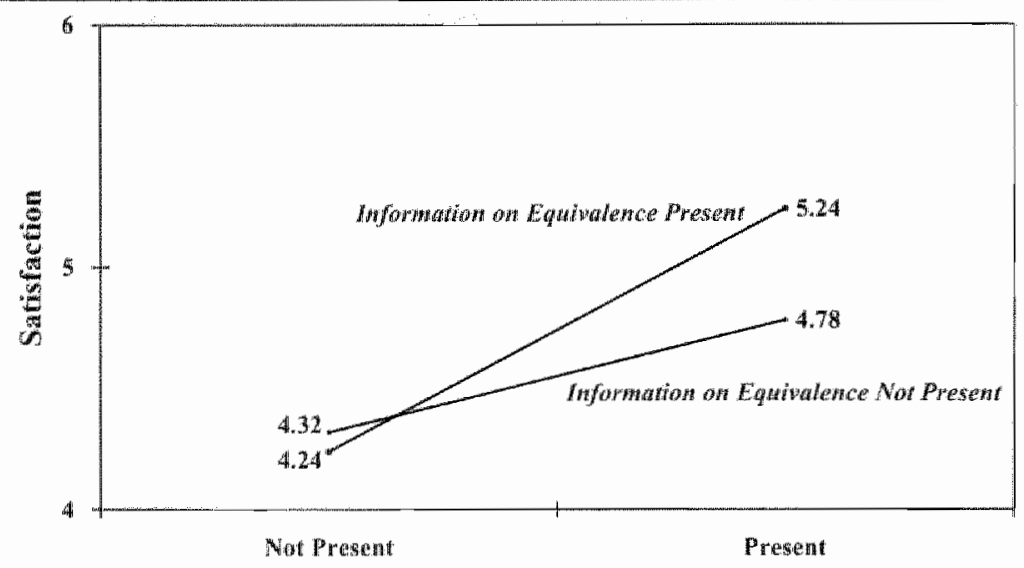

Information on Accountability

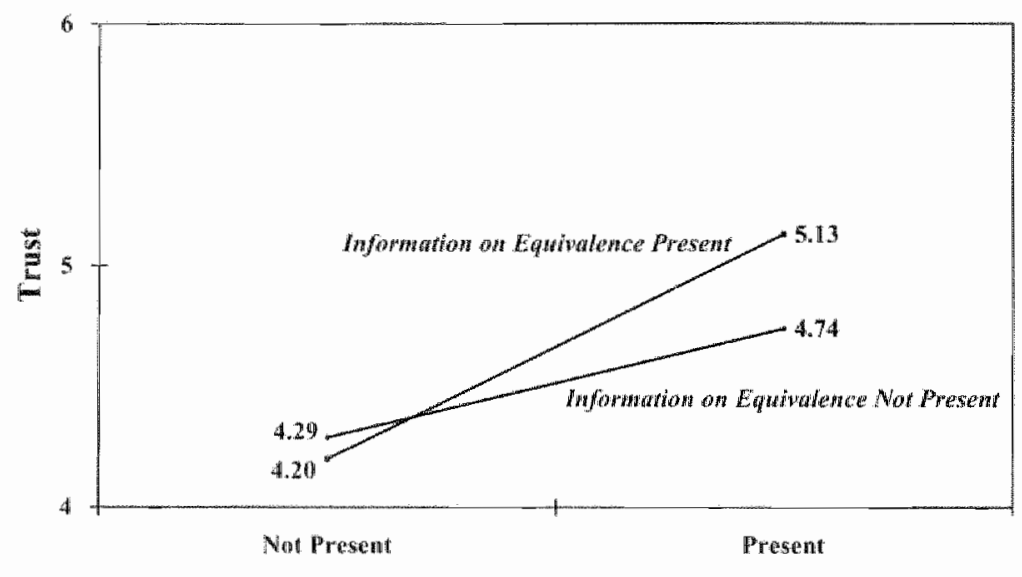

Information on Accountability

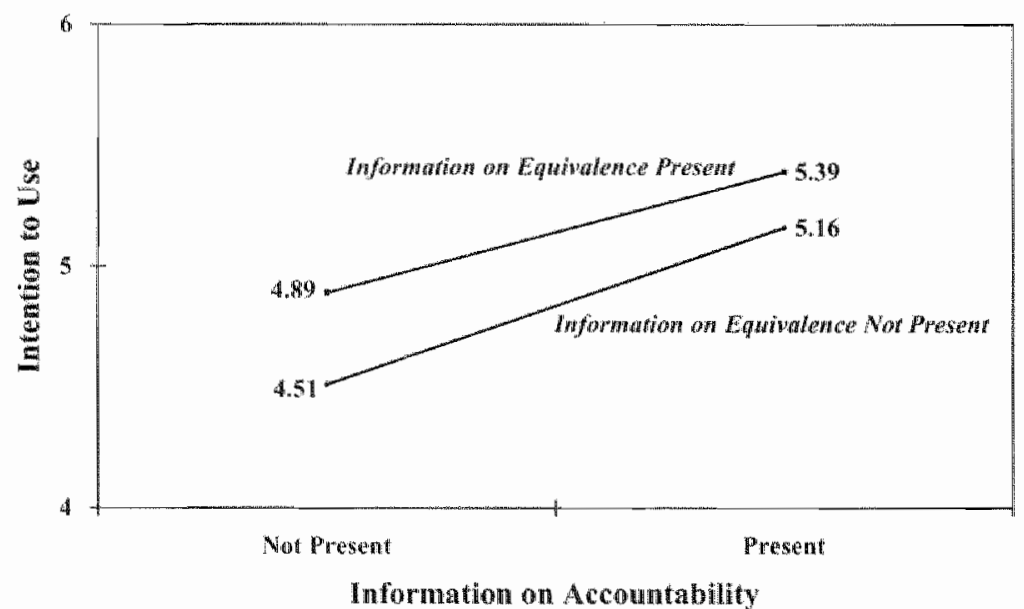


This is depicted graphicaly in figure 3-2: for intention to use the avallable information, the lines run practically parallel to each other, for satisfaction and trust the slopes of the lines are distinctly.

\subsubsection{Hypotheses Testing}

$\mathrm{H}_{3}$ was tested by performing a paired sample t-test across the entire sample of decision-makers to assess whether decision-makers generally are more likely to make predictions about missing information on the basis of other available information than to ignore it. On the basis of this test we fail to reject $H_{1}$ : The prediction strategy of dealing with missing information is used significantly more often than the ignoring strategy $\left(t_{\mathrm{n}}=16.32[\mathrm{p}<0.001]\right)$.

We used univariate contrast analyses to further explore the relationships uncovered by the omnibus test (Neter et al. 1990) and to test hypotheses $\mathrm{H}_{2}$ and $\mathrm{H}_{3}$. From conducting ANOVAs it became clear that the four cells in our factorial design differed significantly on the three dependent variables (Satisfaction: $F_{3.115}=6.34$ $[p=0.001]$; Trust: $F_{3_{1.13}}=5.14[p=0.002]$; Intention to use: $\left.F_{3,114}=4.36[p=0.006]\right)$. With respect to $\mathrm{H}_{2}$, we find that decision-makers who have to make decisions on the basis of an international marketing research report that contains complete information on both cross-national data equivalence and financial accountability of international service investments are significantly more satisfied with, have higher levels of trust in, and have a higher intention to use the available information in the report than decision-makers who have to make decisions on the basis of a report that is missing information on both topics (Satisfaction: $t_{1.5}=3.53[p<0.001]$; Trust: $t_{113}=3.13[p=0.001]$; Intention to use: $\left.t_{134}=3.45[p<0.001]\right]$. Therefore, we fail to reject $H_{2, a}, H_{26}$, and $H_{26}$.

With regard to $\mathrm{H}_{3}$ we find that decision-makers having to make decisions on the basis of an international marketing research report that contains no information on cross-national data equivalence and complete information on financial accountability of international service investments are significantly more satisfied with and have more trust in the available information in the report than decision-makers having to make decisions on the basis of a report that has complete information on equivalence and no information on accountability of investments (Satisfaction: $t_{115}=2.07[p=0.021]$; Trust: $\left.t_{113}=2.06[p=0.021]\right]$ ). No significant difference was found with respect to the intention to use the avallable information in the report (Intention to use: $t_{114}=1.03$

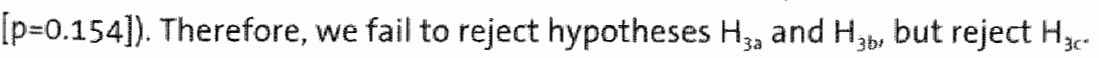

Finally, as was already mentioned in the preceding section, we found a significant two-way interaction effect between both informational topics. Although umesh et al. (1996) caution researchers for using the omnibus F-test for interactions to test for 
differences between cell means, they also indicate that the 2 *2 ANOVA constitutes a special case in that respect ( $c$. Keppel 1991). The interaction effect was significant for both satisfaction with and trust in the available information, but not for the intention to actually use it when making decisions (Satisfaction: $F_{1.115}=6.00[p=0.016]$; Trust: $F_{1,1,5}=5.43(p=0.022]_{i}$ Intention to use: $\left.F_{1,215}=0.20[p=0.656]\right)$. Hence, we fail to reject hypotheses $H_{4 a}$ and $H_{A, 2}$ but reject $H_{B c}$ in other words, the effect of complete information on financial accountability of international service investments is significantly more positive in terms satisfaction with and trust in the available information, for decision-makers who have to make decisions on the basis of an international marketing research report that also has complete information on cros: national data equivalence than for decision-makers having to make decisions on a report with no information on equivalence. For the intention to use the available information no such effect was found.

\subsection{Conclusion}

\subsubsection{Discussion}

In intermational settings companies often will be facing the uncertainty associated with engaging in international business activities. Especially in the services industry international uncertainty is likely to be substantial. Unfamillarity with foreign cultures, customers, and their service practices, combined with the higher perceived purchase risk for foreign services will result in a considerable amount of environmental uncertainty for intermational service providers.

To deal effectively with this uncertainty, service firms depend on the avalability of marketing information. In practice, however, it seems that information hardly ever is complete. In this chapter, we focused on studying the effects of incomplete information on the use of marketing research intelligence in international service settings. More specifically, it was studied how decision-makers in international serwice organizations deal with incomplete information. Furthermore, we looked at the impact of incomplete information on decisionmakers' evaluative judgments in terms of satisfaction with, trust in information and their likelihood of using it for decisionmaking purposes. For conducting the experiment, we focused on wo informational topics of importance for the international marketing of services: information on the level to which cross-national equivalence in research data has been 
established/checked and information on the financial accountability of international service investments.

Consistent with earlier research on human decision-making processes (e.g. Lipshitz \& Strauss 1997), the results of our experimental study show that also in an international service setting incomplete information will not be ignored by decisionmakers. On the basis of other avallable information and knowiedge they will apply a strategy of inferential, assumption-based reasoning. Decision-makers will use other sources of information, such as information that is available in the report, other related studies they are familiar with, and own experiential knowledge to make predictions about the missing information and then combine these inferences with the available information in order to evaluate the overall decision-making usefulness of a report. In this way, the informational uncertainty caused by incomplete information is effectively dealt with.

With regard to the consequences of incomplete information, several interesting results could be deduced from the study. First of all, when distinguishing between complete information on cross-national data equivalence as well as financial accountability of intemational service investments and no information about these issues at all, this resulted in significant effects. In comparison to a situation of incomplete information, complete information on data equivalence and financial accountability of investments led to a significant increase in a decision-maker's satisfaction with the information available in the international research report. Since marketing research is a service provided by a research agency to a client, it is consistent with earlier findings in services marketing research in which positive relationships were found between service quality and customer satisfaction (e.g. Parasuraman et al. 1994; Rust \& Oliver 1994).

Furthermore, complete information also resulted in higher levels of trust of the decision-maker in the available information and a higher intention to use it when having to make decisions about the international services strategy. This also supports previous findings, indicating positive relationships between uncertainty reduction and information quality/completeness on the one hand, and trust and intelligence uselevaluations on the other hand (Deshpandé \& Zaltman 1982, 1984; Moorman et al. 1993; Potter \& Beach 1994).

Information differs in perceived decision-making relevance. It was expected that information on the financial accountability of international service investments would be of higher managerial relevance to decision-makers than information on whether or not research data is internationally equivallent. It was found that presence of accountability information and a lack of equivalence information results in higher satisfaction with and more trust in available information, compared to a situation of 
lacking accountability information and presence of equivalence information. This indicates that decision-makers indeed subjectively tend to differentiate between information relevancy levels. However, no significant effect was found for the intention to use available information. In other words, focusing on relevant information improves decision-makers' evaluative judgments of the information provided, but does not automatically imply that it is going to be used for future decision-making. This suggests that a distinction can be made between attitudinall and behavioral consequences of information presence.

The previous findings suggest that equivalence information is of lower value to decision-makers in international service firms than financial accountability information. Indeed, the presence of accountability information positively influences satisfaction with, trust in, and intention to use the information, as can be concluded from the significant main effects that were found for accountability information. In contrast, no significant main effect was found for equivalence information. However, from testing our last set of hypotheses, it could be concluded that equivalence information is playing a role in combination with accountability information. The positive effect of accountability information on satisfaction and trust turned out to be significantly higher when information on cross-national data equivalence was present in the report compared to when it was missing. With respect to intention to use the information no significant interaction effect was found, providing additional evidence of an attitudinal and behavioral differentiation in evaluative consequences of incomplete information.

\subsubsection{Theoretical Implications}

Part of the strength of a study lies in the recognition of its limitations. This may suggest issues that merit future research. In the first place, the experimental design pertained to an experiment in the field (in one specific service firm with real decisionmakers) under laboratory conditions (artificially constructing the desired conditions by referring to an imaginary research report and decision-making situation). As a consequence, the generalizability of the findings would be limited with regard to reallife marketing decision-making situations. Due to differences in the purpose of the information, decision-makers involved, stage in the internationalization process implying varying degrees of experience, and different service firms in terms of line of business, size, market type, etc. inferences about generalizability should be treated cautiously and explored on the basis of future research in more real-life and other service situations. 
More specifically, the use of an experimental design is subject to inherent limitations pertaining to a possible lack of realism. For instance, our study involved a single sample of each subject group responding to one hypothetical, written case on the basis of limited information. Even though the results of the manipulation checks show successful manipulation of information on cross-national data equivalence and financial accountability of international service investments, differences may arise between simulation and reality, affecting the way in which respondents react. One way of dealing with this may be to provide more real-life scenarios by using physical research reports instead of describing them verbally. In this way, the manipulated conditions are more closely to holistic experience ('gestalt') of an actual marketing decision-making situation.

Furthermore, evaluative judgments in terms of satisfaction, trust, and intention to use have been restricted to a single decision-making episode, thus essentially studied from a static perspective. Future experiments might investigate how these judgments develop over time, in order to examine the impact of missing versus complete information more profoundly and in line with real-life dymamic situations.

Additionally, since conducting a marketing research project is a service, it can be argued on the basis of advances in attribution theory (e.g., Folkes 1994) that decisionmaker perceptions of satisfaction with and trust in marketing research information may be influenced by more than just the presence or absence of information in a research report. Factors relating to the interactive process of conducting an international marketing research project may considerably moderate quality, satisfaction, and trust evaluations by decision-makers. This corresponds to findings of Moorman et al. (1993) which indicate that trust between users and providers of marketing research information is influenced by interpersonal characteristics, such as expertise, integrity, confidentiality, timeliness, and sincerity of providers. Future research should take the impact of such attributions into account. Finally, additional research is needed to address how missing information has an impact on actual behavior rather than a decision-maker's intention to use available information.

\subsubsection{Managerial Implications}

Our results have several managerial implications for providers of international services marketing intelligence. They may enable marketing researchers, when providing their service to clients, to create conditions conducive to effective use of marketing intelligence. First, based on the conclusion that incomplete information is unlikely to be ignored by decision-makers, but will be compensated for by making inferences on the basis of other information, marketing researchers should acknowledge gaps in the 
information they provide. They can anticipate on this by providing additional sources of information that decision-makers can consult in case of incomplete information, such as managerial or research literature and information on the internet.

Next, the results imply that when conducting research for companies active in international service settings, substantial attention should be given to both the implications of conducting cross-national service research as well as to consequences for return on investments. Specifically, marketing managers need to be reassured that reliable comparisons between countries can be made and that attention has been directed at making information internationally equivalent. In addition, they seem to value information about the financial consequences of proposed investuments in the intermational service strategy that follow from the research conducted.

Despite of the importance of providing complete information for the evaluation and use of intelligence, in practice this might be hard to accomplish. Especially in situations when a trade-off between what information to provide has to be made, for example due to budgetary restrictions, intelligence providers could differentiate between relevant and less relevant information. A focus on financial accountability information resulted in higher decision-maker satisfaction with and trust in available information than a focus on cross-national equivalence information. Indeed, it seems that decisionmakers tend to value such, more strategic, information higher.

However, the significant interaction effect that we found between presence of equivalence and accountability information also suggests that additional information, which might be perceived as less relevant, can add value for decision-makers as well. Information on the international equivalence of the information in the international research report has an additional effect on evaluative judgments in terms of satisfaction with and trust in available information if it is presented in combination with financial accountability information. The intention to use information was not significantly influenced by variations in information relevancy. The level to which information is going to be acted upon may depend on other factors, as suggested by Deshpandé and Zaltman $(1982,1984)$. These may include content quality (relating to additional issues such as applied scientific research techniques, data collection methods, sampling procedures), form quality of the research report or its verbal presentation, and providing acceptable information that can be acted upon.

Furthermore, situational factors such as line of service business, firm characteristics, company objectives, level of business experience with foreign countries, etc. will also determine information content and utilization. For example, service firms wanting to export a new service to foreign markets with which they have little experience and which are quite distinct from the home-market will need more and other information than companies planning to provide an existing service to a 
neighbor country with similar characteristics. The former situation would demand more country-specific information on tariff and non-tariff measures, government trade policies, local laws and regulations, competitive activity, market characteristics, cultural background, local standards, specifications, expectations, and distribution systems (Czinkota \& Ronkainen 1995). In the latter case country-specific information may be of lesser importance compared to more company-strategic information, for example on the consequences of service investments. 



\section{Chapter 4}

\section{Effectiveness of International Service Intelligence: A Relational Exchange Perspective $^{1}$}

In essence, marketing research is a service that is delivered by a researcher to an information user, i.e. decision-maker. The acknowledgement of provider-user relational factors, therefore, seems to be worthwhile when studying effectiveness of international service intelligence. Besides intelligence attributes, we, therefore, examine what researcher characteristics drive use of senice intelligence in this chapter, in recent years, academic service research centers have been positioned as feasible research partners and we focus on this specific type of intelligence pravider The results of an empirical study conducted over two years suggest that user satisfaction is central to establishing decision-maker trust, as well as promoting the use of the information. In both years, quality of content and researcher characteristics were ldentified as key drivers of satisfaction in the initial stage of the relationship between the service research center and the client firm, information use is also driven by the political acceptability of the information. Trust in the researcher did not seem to have an effect on information use. The results of the second year, however, suggest that particularly trust becomes a crucial factor as the relationship develops for it then directly impacts the use of information provided by academic service research centers.

\footnotetext{
This chapter is largely based on: Birgelen, M.J.H. van, J.C. de Ruyter \& M.C.M. Wetzels (2001). What Makes Service Research Centers Effective?", Journal of Service Research, 3 (3) Forthcoming
} 


\section{Effectiveness of International Service Intelligence: A Relational Exchange Perspective}

\subsection{Introduction}

Effective use of marketing information is crucial for service providers in today's competitive environment in which geographical boundaries are rapidly converging (Menon \& Varadarajan 1992). The task of gathering information is commonly the responsibility of internal research departments or commercial research agencies. Recently, university-based service research centers have profiled themselves as a new research partner. One unique selling point of these centeirs is that clients have direct access to scientific knowledge and expertise. Alternatively, partnering with firms yields the benefits of access to testing grounds and additional funding for academic service research centers.

So far, however, virtually nothing is known about factors determining the performance of these service research centers. The question what makes service research centers effective has been left unanswered. This seems to be an important omission in the service research literature. In this chapter, we attempt to formulate an answer. It is structured as follows. First, the construct of intelligence use is conceptualized briefly and a number of possible antecedents are discussed.

Subsequently, we develop a new conceptuat model on academic marketing intelligence use and formulate several research hypotheses. Finally, the results of an empirical study, conducted in an international business-to-business service setting at two different points in time, are presented and a number of theoretical and managerial implications are provided.

\subsection{Marketing Intelligence Use}

A multitude of studies have focused on the use of marketing intelligence by organizations (e.g., Deshpandé \& Zaltman 1982, 1984, 1985, 1987; Krishnan 1989; Mattz \& Kohli 1996; Menon \& Wilcox 1994; Moorman et al. 1992, 1993). A widely used conceptualization of knowledge use distinguishes between instrumental use, conceptual use, and symbolic use (Menon \& Varadarajan 1992). instrumental use is the application of knowledge to solve a particular problem or make particular 
decisions (Deshpandé \& Zaltman 1982). Information can also be used for general enlightenment rather than for taking actions. This is referred to as conceptual use (Deshpande \& Zaltman 1982), which Menon and Wilcox (1994) classify as knowledgeenhancing use. Through conceptual use, knowledge and understanding of the issues under study will be changed. Finally, information can also be distorted beyond its correct intent and used symbolically (Feldman \& March 1981; Menon \& Varadarajan 1992). Symbolic use occurs when intelligence is misused by taking conclusions out of their context and disclosing only those that confirm an executive's predetermined positions, by oversimplifying findings, and/or by consciously ignoring any accompanying caveats or assumptions that may weaken the findings. Other forms of symbolic use are using findings to legitimize and sustain previously held dispositions or distorting findings to justify actions taken for other reasons.

in addition to conceptualization issues, it seems relevant to pay attention to factors that influence the use of intelligence by marketing decision-makers. In order to facilitate this we conceive of the provision of market information as a service. When taking such an approach, the well-known distimction between technical and functional service quality applies. Technical quality refers to the (international) marketing information provided. while functional quality relates to the way in which this information is being delivered to the client. Deshpandé and Zaltman (1982) identified a range of technical and functional factors that relate to the instrumental use of market research intelligence. Examples are the quality of the contents (techmical) and the interaction between information provider and client (functional). This interaction, in turn, is related to certain interpersonal characteristics of the researcher, such as integrity and expertise (Moorman et al. 1993). Based on path analyses, Deshpandé and Zaltman (1982) concluded that higher levels of perceived content quality, perceived ability to take actions on the research, and political acceptability of recommendations resulted in higher instrumental use of intelligence.

The causal relationship between quality of the information format and instrumental use was found to be relatively weak. Furthermore, higher levels of perceived interaction between research supplier and client were also positively related to instrumental utilization of market research information.

These findings apply to a business to consumer context. When extending their study to a business-to-business setting, different results were reported by Deshpande and Zaltman (1987). Apparently, the nature of industrial settings has specific characteristics that distinguish them from consumer goods settings and that translate to the role of marketing information and the factors influencing its use. According to Webster (1978) these are greater functional interdependence, greater technical praduct complexity, stronger buyer-seller interdependence, and greater buying 
process complexity. This seems to justify further systematic and empirical analyses of marketing intelligence utilization in business-to-business service settings The level of trust that organizations have in research providers also has been shown to influence intelligence use (Maltz \& Kohli 1996; Moorman \& Austim 1995; Moorman et al. 1992). Moorman et al. (1993) state that the level of trust in producers of intelligence will determine mere possession of information or actual use. The level of trust was also found to influence perceived quality of user-provider interactions, the level of researcher involvement, and the level of user commitment to the relationship (Moorman et al. 1992). Taking aforementioned findings into account, we now develop a conceptual framework of academic inteligence use in international business marketing setting by developing a number of research hypotheses.

\subsection{Development of a Conceptual Framework and Research Hypotheses}

In this chapter, we define academic intelligence utilization as the extent to which a marketing decision-maker uses information disseminated by an academic research center to understand the international environment and to make and implement marketing decisions. We posit that the actual use of information is determined by satisfaction with the outcome and process of the research project as well as trust in the researcher partner. Furthermore, in accordance with previous findings in the relationship marketing literature on services (De Ruyter \& Wetzels 2000), we expect that trust in the researcher depends on satisfaction with the academic research project. In turn, satisfaction will be causally related to four technical service quality constructs, namely quality of contents, quality of form, actionability, and political acceptability of information, and one functional service quality construct, namely researcher interpersonal characteristics. Our conceptual model is rendered in figure 41.

Satisfaction can be described as the consumer's cognitive and affective evaluation of the product or service that has been provided by a specific supplier. A relationship between two partners can only continue to exist when a certain level of trust between both parties has developed (Wetzels et al. 1998). Moorman et al. (1992, p. 315) define trust as "a willingness to rely on an exchange partner in whom one has confidence" and found that trust has a positive effect on intelligence utilization. Furthermore, satisfaction is likely to lead to acceptance and use of the academic intelligence for marketing decision-making purposes. Consequently, we posit the following hypotheses: 


\section{Figure 4-1: Conceptual Model of International Service Intelligence Use}

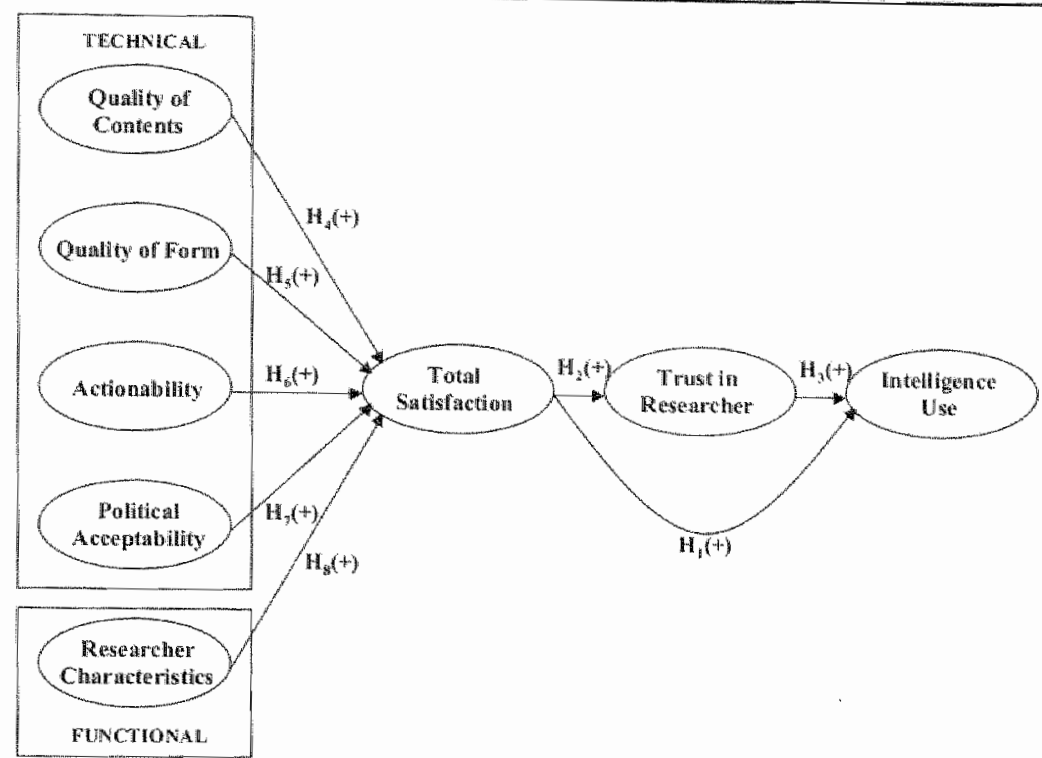

$H_{1}$ : There will be a positive relationship between total satisfaction with the academic research project and academic intelligence use.

$H_{2}$ : There will be a positive relationship between total satisfaction with the academic research project and trust in the academic researcher.

$H_{3:}$ There will be a positive relationship between trust in the academic researcher and academic inteligence use.

Satisfaction is a subjective evaluation that follows the experience of quality (de Ruyter et al. 1997). Technical service quality consists of quality of contents, quality of form, actionability, and political acceptability of the information disseminated. Quality of contents refers to issues that form the perceived overall quality of a marketing research project, such as applied research techniques, apropos use of scientific measurements, structure of data collection, and price-value trade-off.

Menon and Varadarajan (1992) suggest that a receiver's perception of intelligence quality influences the degree to which (s)he acts on it. Deshpande and Zaltman (1982, 1984) found a positive effect of quality of contents on use of the information. As for all service quality-related constructs, we expect the relationship between quality of contents and intelligence use to be mediated by decision-maker total satisfaction with 
the academic research project, resulting in the following hypothesis:

$H_{4}$ : There will be a positive relationship between quality of contents and total satisfaction with the academic research project.

In addition to quality of contents, quality of form of the research report and/or its presentation might have an effect on academic intelligence use. The specific physical form of marketing research has been shown to have an impact on use (Deshpande \& Zaltrnan 1982, 1984). We hypothesize the following relationship:

$H_{5}$ : There will be a positive relationship between quality of form and total satisfaction with the academic research project.

To increase the likelihood of intelligence use by decision-makers information should be actionable. The value of research intelligence increases if managers perceive the intelligence provided as actionable (Deshpandé \& Zaltman 1982). Actionability can be described as the extent to which the intelligence and recommendations are perceived to be implementable into practice and was found to positively influence intelligence use as well (Deshpandé \& Zaltman 1982, 1984). The following hypothesis is formulated:

$H_{\text {G: }}$ There will be a positive relationship between actionability of academic intelligence and total satisfaction with the academic research project.

Political acceptability of academic marketing intelligence can be described as the compatibillty of the information with company objectives. It refers to the fact whether information is tuned to internal policies and objectives. According to Kohli and Jaworski (1990), responsixeness of intelligence utilization may be function of factors such as the political acceptability of intelligence and the extent to which it challenges status quo. In support of this, Deshpandé and Zaltman $(1982,1984)$ found a positive effect of political acceptability on inteiligence use. We expect that higher levels of political acceptability of academic intelligence results in higher total satisfaction levels. and thus ultimately in higher intelligence use:

$H_{7}$ : There will be a positive relationship between political acceptability of academic intelligence and total satisfaction with the academic research project.

Finally, functional service quality is mainly determined by interpersonal 
characteristics of the researcher(s) conducting the study. Important qualities are for example expertise, integrity, flexibility, and confidentiality. Moorman et al. (1993) found an empirical causal linkage between interpersonal qualities and decision-maker trust in the researcher. We posit that this relationship will be mediated by total satisfaction with the research project:

$H_{8^{*}} \quad$ There will be a positive relationship between researcher characteristics and total satisfaction with the academic research project.

In the next section, we report on an empirical test of our conceptual framework.

\subsection{An Empirical Study}

\subsubsection{Research Design and Data Collection}

An academic service research center in the Netherlands has been conducting an international marketing research project for a multinational office equipment manufacturer. The project, similarly conducted in 11 countries in which the manufacturer has operating companies, involves a large-scale international customer satisfaction survey with respect to the multinational's after-sales services. Our study focuses on the effectiveness of the inteligence generated by this international research project. More specifically, in all 11 countries, data was collected on each of the project's technical and functional service quality level, decision-maker total satisfaction with the project, trust in the local researcher conducting the project, and the use of the intelligence provided. The data was collected with respect to two consecutive years in which the international customer satisfaction research project has been conducted. This two-year period of data collection on intelligence effectiveness enables us to validate our conceptual model over time and compare empirical relationships between both years. Our sample of decision-makers came from the multinational's upper and middle-lower management levels.

A mail questionnaire was designed, consisting of multiple items that operationalize the constructs in our conceptual model. Each item was formulated as a statement on which respondents could react by using a 7-point Likert-type scale $11=$ strongly disagree, $7=$ strongly agree). In year 1 , a total number of 80 questionmaires were mailed to managers across several operating companies of the multinational manufacturing organization. In year 2,87 questionnaires were mailed. Questionnaires 
were accompanied by a cover letter and a postage-paid return envelope. Initially, 68 and 78 respondents participated in our study for year 1 and 2, respectively. However, on the basis of control questions assessing respondents' job titles and whether or not they were familiar with the information from the research project, 3 respondents were excluded from our sample for year 1 and 5 respondents for year 2, since they could not be expected to be in an actual decision-making position involving the project. Consequently, the final sample consisted of 65 respondents for year 1 and 73 respondents for year 2 , resulting in response rates of $81 \%$ and $84 \%$, respectively. Respondents were mainly working in business analytical positions (year 1: $5 \%$, year 2 : 6\%; e.g. business analyst), strategic decision-making positions (year 1: 11\%, year 2 : $13 \%$; e.g. strategic planner), product-related positions (year 1: $22 \%$, year $2: 18 \%$; e.g., product manager), and service operations positions (year 1: 48\%, year 2: 53\%; e.g., service manager).

\subsubsection{Questionnaire Development}

In addition to our literature review, the research instrument was developed on the basis of interviews with users of marketing research information. These respondents were excluded from the actual study, since this could evoke test-retest biases. The interviews also yielded a number of items that needed to be adapted in the final questionnaire. The constructs relating to the technical service quality of the academic research project (quality of contents, quality of form, actionability, and political acceptabillity) were operationalized on the basis of validated scales previously used by Deshpandé and Zaltman (1982). Quality of contents was measured by 7 items, quality of form by 4 items, actionability by 6 items and political acceptability by 4 ittems. In order to measure the researcher interpersonal characteristics, representing functional quality. 5 items based on Moorman et al. (1993) were used. The 3 items relating to total satisfaction with the research project were developed specifically for this study. The items focused on measuring satisfaction with the contents, the presentation, and the researcher, and thus on research project outcome and process. Trust in the researcher was measured by 5 items on the basis of a scale by Moorman et al. (1992). Finally, academic intelligence use was operationalized by 7 items on the basis of a scale by Maltz and Kohli (1996). Table 4-1 presents a number of sample items for each of the constructs. 


\subsubsection{Data Analysis}

Before testing our hypotheses, we first assessed the level of internal consistency of the scales by performing reliability analyses. Table 4-1 presents the Cronbach's Alpha reliability scores of the various scales. As can be seen in table 4-1, for both years the reliability scores approximate or exceed the desired threshold value of 0.70 , except for political acceptability in year 1.

Table 4-1: Sample Items and Construct Reliabilities

\begin{tabular}{|c|c|c|c|}
\hline Construct & Sample Item ( $1=$ Strongly Disagree, $7=$ Strongly Agree) & $\alpha(y+1)$ & $\alpha(y+2)$ \\
\hline $\begin{array}{l}\text { Qunalicy of } \\
\text { Contents }\end{array}$ & $\begin{array}{l}\text { The nesearch techniques we applied appropriately } \\
\text { The information provided was worth the money spent } \\
\text { The results of the research project can be tosed very well to solve } \\
\text { current problems within } X X X\end{array}$ & 0.78 & 0.75 \\
\hline $\begin{array}{l}\text { Quality of } \\
\text { Form: }\end{array}$ & $\begin{array}{l}\text { There were too many tables graphs/statisics in the presertation } \\
\text { The analysis of the data in the presentation was more complex than } \\
\text { necessary }\end{array}$ & 0.82 & 0.73 \\
\hline Actionability & $\begin{array}{l}\text { Besides statistical date the report prowides also explicit } \\
\text { mecommendations for action } \\
\text { The recommend thions are asy to put into atect } \\
\text { The infomation was on the for taking pending decisions }\end{array}$ & 0.82 & 0.76 \\
\hline $\begin{array}{l}\text { Political } \\
\text { Acceptability }\end{array}$ & $\begin{array}{l}\text { The recommendations seemed to be organization-potitically } \\
\text { acceptable io me } \\
\text { The recommendations atre in line with the prevalling beliefs at } \times X X\end{array}$ & 0.49 & 0.73 \\
\hline $\begin{array}{l}\text { Resenrchar } \\
\text { Chanactenstics }\end{array}$ & $\begin{array}{l}\text { The researcher has a high level of expenise } \\
\text { The researcher seems to have a great deal of integrity } \\
\text { The researcher can be expected to keep confidenial what he/she } \\
\text { learns about our organizathon beyond the specific research projed }\end{array}$ & 0.86 & 0.87 \\
\hline $\begin{array}{l}\text { Total } \\
\text { Salisiaction }\end{array}$ & 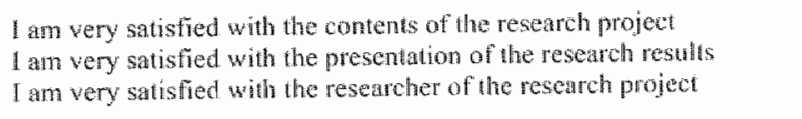 & 0.87 & 0.77 \\
\hline $\begin{array}{l}\text { Trus in } \\
\text { Rescarcher }\end{array}$ & $\begin{array}{l}1 \text { trust the researcher to do things my deparment can not do } \\
\text { I gencrally do trist the researcher }\end{array}$ & 0.65 & 0.80 \\
\hline $\begin{array}{l}\text { Interligence } \\
\text { Whe }\end{array}$ & 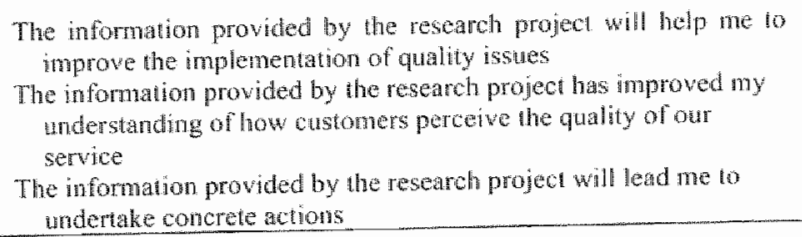 & 0.79 & 0.36 \\
\hline
\end{tabular}

We chose not to explicitly assess the psychometric properties of the measurement instruments in this explorative study, as the sample size in both years is insufficient for the use of exploratory factor analysis or confirmatory factor analysis. Therefore, we 
opted for aggregating the items for each construct and then correcting for measurement error by fixing the path from the indicator to the construct $\left(\Gamma^{*}\right.$ and $\left.\mathbb{I}^{-4}\right)$ by coefficient $\alpha$ and the error variance of the indicator by 1 minus coefficient $\alpha$. This simpler model allows us to estimate the structural model at a more favorable ratio of sample size to free parameters than the measurement model. Netemeyer et al. (1990) report that this approach basically yields the same results as a latent variable model with multiple indicators. The main advantage is that it deals with the sample size problems occurring with the use of structural equation modeling with multiple indicators with a relatively small sample size.

One major weak point of this approach is that quality of construct measurement can not be explicitly assessed. A solution for this problem is using confirmatory factor analysis (measurement model) in the first stage of the analysis (cf. Anderson \& Gerbing 1988). As already mentioned, in the present study this is not possible due to the small sample sizes. Therefore, in order to gain more insight into construct validity, bivariate correlation analyses were performed. The results of these analyses for year $\mathbb{1}$ and year 2 are presented in table 4-2. Overall, table 4-2 shows that constructs indeed are significantly correlated in the direction being hypothesized, except for political acceptability and satisfaction in year 1. Furthermore, we found that all correlation coefficients were statistically significant different from unity.

Table 4-2: Bivariate Construct Correlations"

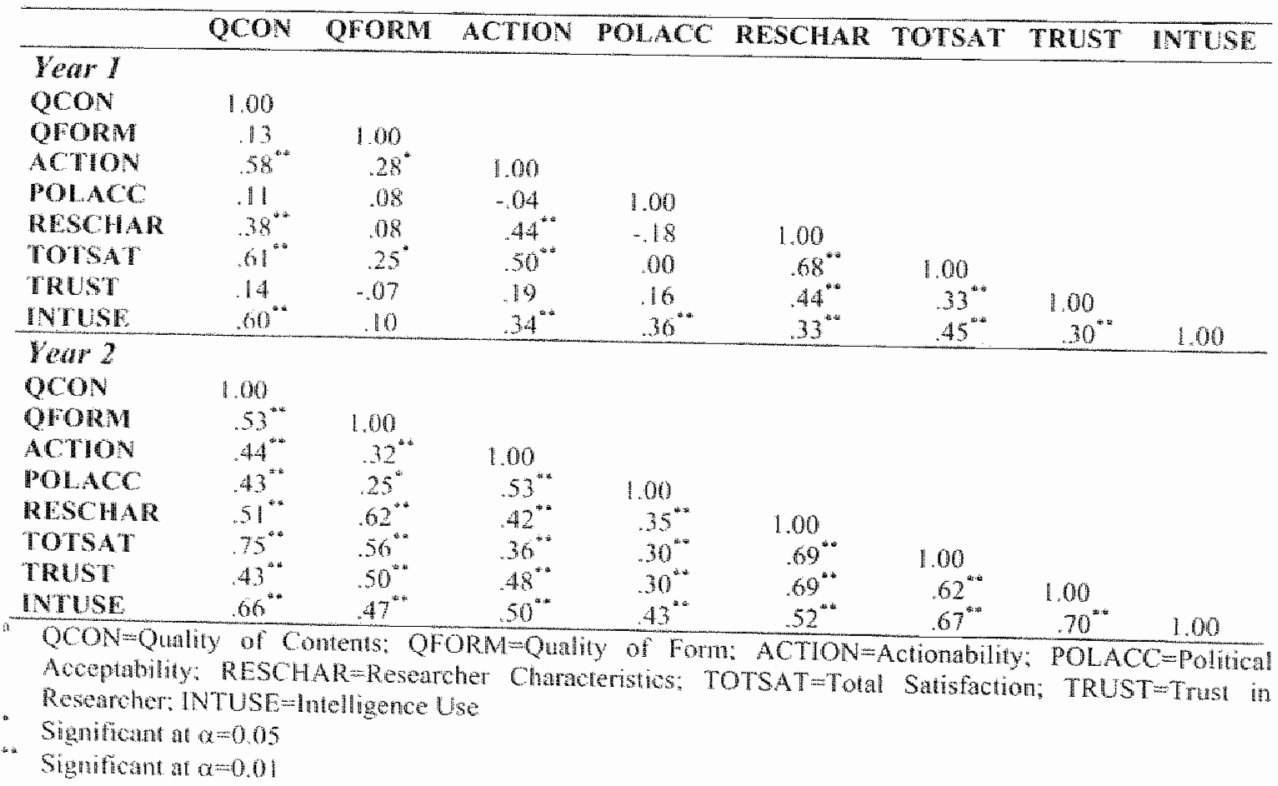


Satisfaction and related measures are prone to extreme skewness. Since LISREL relies on strong assumptions of normality we conducted additional analyses to check the distribution of the variables. Although some variables (quality of contents (year 1 and 2), quality of form (year 1 and 2), total satisfaction (year 1 and 2), intelligence use (year 1)) deviated significantly from a normal distribution using a Kolmogorov-Smirnov test this might be due to sample size. A more detailed evaluation revealed that these variables were significantly negatively skewed. However, inspection of the expected normal probability plot and the expected normal probability plot revealed that the magnitude of skewness was only moderate. These results were corroborated by inspection of the expected normal probability plot (Q-plot) of the standardized residuals of the models, which showed a straight line on a slope larger than $45^{\circ}$ for both models. Based on these analyses and the corrective actions taken concerning limited sample size we feel confident to test the hypotheses using our data.

\subsubsection{Hypotheses Testing}

In order to test the hypotheses we formulated, structural equation modeling with LISREL ${ }^{\text {B }} 8$ was used. We used LISREL ${ }^{\text {D }}$ to obtain maximum likelihood estimates of the path coefficients. As the $\chi^{2}$-value is not independent of sample size, various fit indexes have been developed that are supposedly independent of sample size (Hu \& Bentler 1995). Among these, the Tucker-Lewis Index (TLI) and the Comparative Fit Index (CFI) seem to be relatively unaffected by sample size.

The fit of our proposed model turned out to be good for year 1, as well as for year 2 . For year 1 , the fit statistics are as follows: $x_{(12)}^{2}=14.28(p=0.28), G F=0.95, A G F \mid=0.85$, RMSEA $=0.036, T L I=0.95, C F l=0.98$. For year 2 , these statistics are: $\chi_{(11)}^{2}=12.89(p=0.30)$, $\mathrm{GFI}=0.96, \quad \mathrm{AGFl}=0.86, \quad \mathrm{RMSEA}=0.044, \mathrm{TLI}=0.98, \quad \mathrm{CFI}=0.99$. All measures closely approximate or exceed the recommended cut-off vallues. The hypotheses can be tested by inspecting the path coefficients. The empirical relationships for the consecutive years are rendered in figures $4-2$ and $4-3$.

As can be concluded from these figures, hypothesis 1 is supported for both years by a significant positive relationship between total satisfaction with the academic research project and academic intelligence use by decision-makers. Decision-makers that in total are more satisfied with the research project are more likely to use the information for marketing decision-making purposes. Furthermore, in support of hypothesis 2, we found for both years a significant positive relationship between total satisfaction and the level of decision-maker trust in the researcher conducting the project. This indicates that decision-makers that are more satisfied are likely to develop a higher level of trust in the academic researcher. 
Figure 4-2: Causal Modell of International Service Intelligence Use (Year 1)

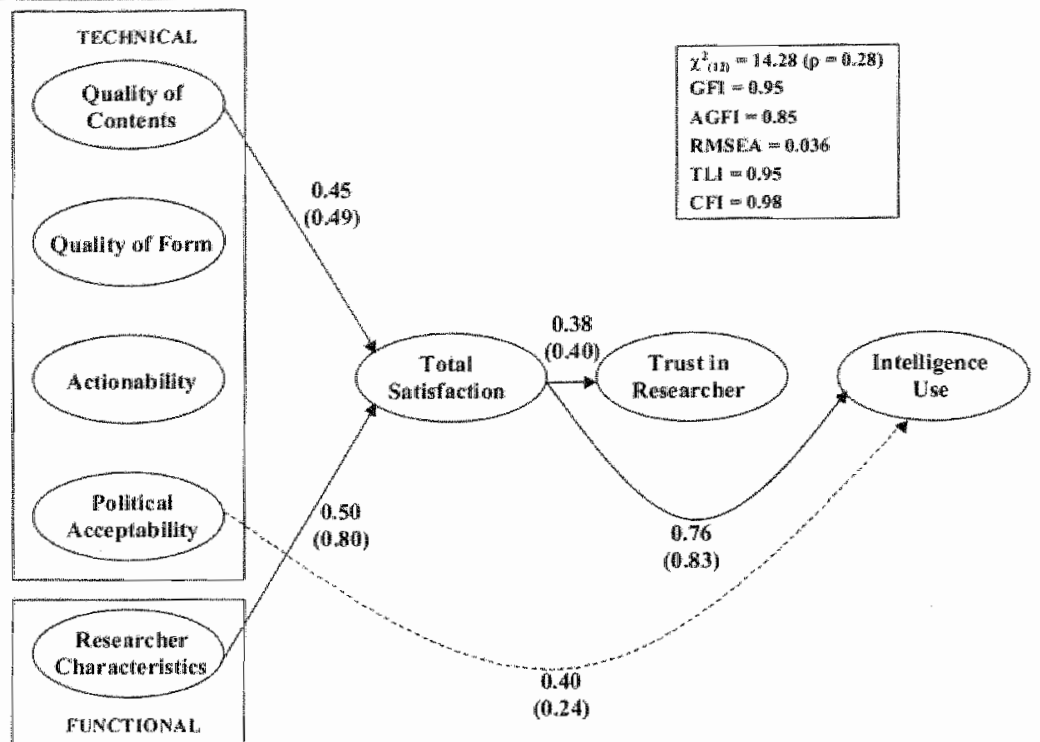

"Only significant standardized path coefficients are shown $(\alpha=0.05)$

b Unstandardized patls coefficients betwean parentheses

\section{Figure 4-3: Causal Model of International Service Intelligence Use (Year 2)}

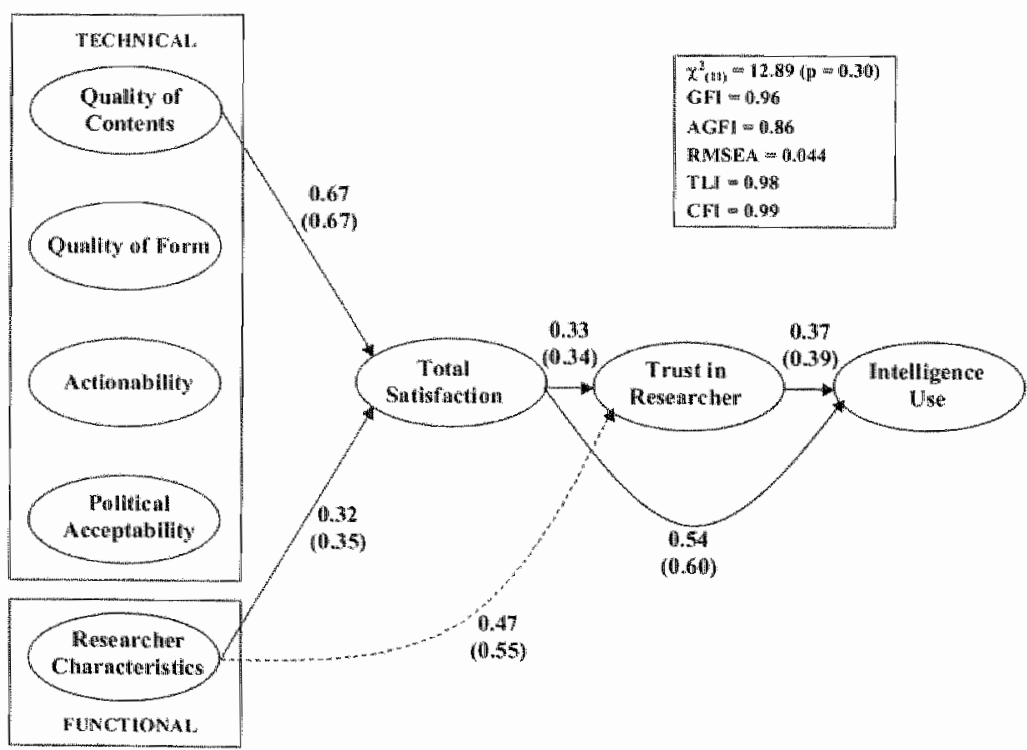

Only significant standathed path coethicients are shown $(\alpha=0.05)$

Unstandardized path coefricients between perentheses 
With respect to hypothesis 3 , no consistent relationship between trust in the researcher and academic intelligence use was found. For year 1 there was no significant relationship. whereas for year 2 higher trust in the researcher indeed results in higher academic intelligence use. Therefore, hypothesis 3 is rejected for year 1 and supported for year 2 .

Concerning the factors that influence total satisfaction with the academic research project the following conclusions can be drawn from our empirical study. Hypothesis 4 is supported by the data for both years: a significant positive relationship was found between quality of the contents of the academic research project and total satisfaction. The more positive a decision-maker evaluates the actual contents of the project, the more satisfied he or she will be in total. For both years we found no significant relationships between quality of form, actionability, and political acceptability on the one hand and total satisfaction with the academic research project on the other hand. Therefore, hypotheses 5,6 , and 7 are not supported by our study. In support of hypothesis 8 , however, it was found for both years 1 and 2 that interpersonal characteristics of the researcher indeed are positively related to total satisfaction with the project. The more positive the interpersonal characteristics of the researcher are evaluated, the more satisfied the decision-maker is with the academic research project in total.

The non-significant relationships that were found for quality of form, actionability, and political acceptability of the intelligence gave rise to some further analyses. Inspection of the modification indices suggested two additional relationships in our model, which have not been hypothesized a-priori. on the basis of these indices, it could be concluded for year 1 that, in support of Deshpandé and Zaltman (1982), political acceptability of the academic intelligence was directly and positively related to its use for marketing decision-making purposes. The more the information is perceived as acceptable for the service providing firm and in agreement with company objectives, the more likely it will be used. Furthermore, in accordance with Moorman et al. (1993), we found a significant positive relationship for year 2 between researcher interpersonal characteristics and decision-maker trust in the researcher. The more positive the academic researcher interpersonal characteristics (e.g., expertise, integrity, and flexibility) are evaluated, the more the researcher will be trusted by users of the intelligence disseminated. 


\subsection{Conclusion}

\subsubsection{Discussion}

Our study yields a number of interesting findings. First of all, differences over time were found. This emphasizes the dynamic nature of relationships between the center and its clients. Initially, the results suggest that it seems important to demonstrate an in-depth understanding of the client"s situation and provide information that is in accordance with the client company objectives. As the relationship develops, trust in the researcher becomes increasingly important. For both years, satisfaction had a significant, positive effect on trust in the researcher. This provides support for our reasoning that satisfying clients is important for academic providers of research intelligence, both from a short-term as well as a long-term perspective. Satisfied users are more likely to actually use the intelligence for service marketing decision-making purposes. Moreover, satisfaction increases decision-maker trust in the researcher, which ultimately becomes an important factor for achieving higher levels of intelligence use. An insight into the drivers of satisfaction, therefore, seems relevant. For both years, quality of contents is a key driver. The same holds for researcher interpersonal characteristics, such as expertise, integrity, and flexibility. For both years, these characteristics were also found to be positively contributing to user satisfaction.

The results of another recent study conducted by the authors which utilized the same conceptual model tested for commercial research agency/user dyads suggest that there are differences with respect to the specific setting in which marketing intelligence is investigated (see van Birgelen et al. 2000). 怆 was found that, in addition to quality of contents and research agency characteristics, significant positive effects of quallty of form and actionability on user satisfaction exist. Furthermore, quality of contents had a direct positive effect on intelligence use and, as was also found in the present study for year 1, trust did not significantly affect intelligence utilization. Comparison of the results of both studies suggests that for the evaluation of commercial agencies other factors seem to be taken into main consideration.

\subsubsection{Theoretical and Practical Implications}

An essential attribute of academic research is the acknowledgement of its shortcomings. In this way, potential issues that merit future research efforts may be identified. Our findings are based on one specific setting. Extension to other settings is. necessary. Also, more insight should be obtained into the underlying reasons for 
differences in effectiweness between academic and commercial research centers. In addition, our study suffers from a number of measurement shortcomings. Reliability and validity issues should be addressed in future research. In addition, despite the amount of variance in total satisfaction explained by our service quality constructs, the inclusion of additional variables could improve the model. Such variables might include user organizational characteristics, such as organizational structure and culture, or inter-organizational characteristics, such as user or researcher power in the dyad (Moorman et al. 1993). Finally, future research should take different types of information utilization into account. In the present study, we focused on a combination of instrumental and conceptual use. By making an explicit differentiation between utilization types, further insights into the objectives for which information can be used and factors influencing these usage types could be developed. For example, it can be expected that the level to which information can be acted upon (actionability) might become more important when the main objective of the information is strategy determination (instrumental use). On the other hand, when intelligence is intended to legitimize or justify previous actions (symbolic use). the effect of political acceptability might be quite strong.

Our findings have several practical implications for academic service research centers. Academic research centers should continuously monitor satisfaction levels of the clients they conduct research for. Evaluative meetings during as well as at the end of a research project are needed for assessing client satisfaction and for identifying potential issues for improvement. Furthermore, our results suggest that the adoption of a relationship oriented perspective is essential. When the research project was conducted for the first time, information-related factors had a direct effect on intelligence use. Service research centers, therefore, need to develop a thorough understanding of the client organization's specific decision-making situation, existing belliefs, budgets, and resource allocations. Over time, establishing trust by focusing on account management skills seems essential. This implies that, in research personnel selection and training procedures, it is desirable to explicitly focus on traits like integrity, flexibility and overall customer service orientation. Researchers should adhere to high standards, maintain objectivity in the research process, be aware of the confidential nature of the information they provide, and possess the knowledge and technical skills to do their job adequately. In order to satisfy clients, it is important for academic researchers to keep in mind that they seem to be evaluated on different factors in comparison to commercial research agencies. Without implying they are not important, factors as the way in which results are presented, actionability and political acceptability of the information seem to be less relevant for service research center effectiveness. It seems to be more important to focus on quality of the contents of an 
academic research project by offering transparency and background information on research techniques, data collection methods, argumentation and conclusions and creating a perception of value for money. 


\section{Chapter 5}

\section{The Impact of Attitude Strength on International Service Intelligence Effectiveness}

In this chapter, we zoom in on the user of information and adopt an attitudinal perspective on the effectiveness of international service intelligence, More specifically. we aim at predicting intentions of selectively using service customer satisfaction intelligence from decision-maker attitude strength. The results suggest that two dimensions of attitude strength, commitment and embeddedness, contribute directly and indirectly to selective international service intelligence use. Indirect effects occur through cognitive processes and selective judgment of satisfaction related information. 


\section{The Impact of Attitude Strength on International Service Intelligence Effectiveness}

\subsection{Introduction}

Organizations place great emphasis on satisfying their customers nowadays. A recent quote from a well-known fortune 500 company's mission statement illustrates this: "At Motorola Customer Satisfaction is our top priority". An emerging body of academic investigations has established the economic benefits of customer satisfaction empirically (e.g., Anderson 1996; Rust et al. 1995; Wetzels et al. 1998). These include customer retention, customer commitment, positive word of mouth, and higher profitability.

As a consequence, organizations search for ways to monitor their customers" satisfaction levels and collect information on the "customer voice". Considerable portions of marketing budgets are often spent on conducting customer satisfaction surveys. However, it has been argued that competitive advantage does not lie in access to intelligence but in the ability to effectively use it (Menon \& Varadarajan 1992; Moorman et al. 1992). In the modern context of tremendous information availability through advances in information technology and research practice, use of customer satisfaction-related information does not always appear to be optimal. Decisionmakers get frustrated when it turns out that, despite of repeated measurement and attention for quality-related issues, no changes in customer satisfaction levels are evident (Mulder 1999). This could cause managers to cast doubt on the topic of customer satisfaction. Moreover, customer satisfaction research is often conducted on behaif of managers who have a marketing (research) background and who occupy positions in which customer orientation is pivotal. Hence, they have often developed relatively strong views on how this type of research should be conducted (Deshpandé \& Zaltman 1982). This could easily lead to mistrust in, selective use, or non-use of information.

Given the economic cost of customer satisfaction research and the tremendous opportunity cost of not using information, it seems worthwhile to further develop our understanding of determinants of customer satisfaction intelligence use. Over the years, marketing intelligence utilization has received substantial attention in academic and managerial literature (e.g." Deshpandé \& Zaltman 1982, 1984, 1987; Lee et al. 1987; Maltz \& Kohli 1996; Moorman et al. 1992, 1993; Raphael \& Parket 1991). The majority of this literature explains significant variance in marketing information use 
with external factors, such as research quality, actionability and acceptability of results, interpersonal characteristics of the researcher, and organizational characteristics. However, with the exception of a few studies (e.g., Lee et al. 1987, 1997; Perkins \& Rao 1990), little research efforts have contributed to internal, decision-maker-related factors and of these studies none focused explicitly on customer satisfaction-related information.

Based on findings in social and organizational psychology and behavioral decisionmaking research, we posit that internal dispositions about customer satisfaction can be expected to co-determine intelligence use. Strong evidence was found for the effect of attitude strength on behavior and behavioral intentions (see Krosnick and Petty (1995) for an overview). In addition to a strongly held position, we argue that mediating factors, such as cognitive elaboration on customer satisfaction and selective judgment of information, will impact the use of information. The chapter is structured as follows. First, a review will be given of attitude strength in order to develop a more profound understanding of this theoretical concept. Next, cognitive and behavioral implications of attitude strength will be introduced. This background review will then serve to develop a conceptual framework on the consequences of attitude strength for intentions of using satisfaction-related information. After presenting the results of an empirical study, the chapter will conclude with a discussion of several theoretical and managerial implications.

\section{2 Attitude Strength: An Overview}

Individuals tend to differ in the way they relate to specific issues. In the social and economic psychology literature, among others, the term attitude is used to describe these differences. A basic definition of an attitude is: "Attitude is a psychalogical tendency that is expressed by evaluating a particular entity with some degree of favor or disfavor" (Eagly \& Chaiken 1993, p. 1). Attitudes can differ in strength between persons. Attitude strength, a concept frequently used in attitude research and theory (Pomerantz et al. 1995), is traditionally studied in settings of socially significant and controversial issues (e.g. Krosnick et al. 1993).

A review of the literature fails to give a predominating conceptual definition of this concept. Nonetheless, an overall consensus can be found: strong attitudes are described as resulting in selective cognitive processing and will be resistant to change, persistent over time, and highly predictive of behavior (Abeison 1988; Chaiken et al. 1995; Eagly \& Chaiken 1993, 1995; Krosnick et al. 1993; Olson \& Zamna 1993). Krosnick 
and Petty (1995) also refer to the strong impact of attitude strength on behavior and the processing of information. In our perspective, both characteristics of attitude strength, its impact on behavior and information processing, contribute strongly to the relevancy of this concept for a study on intentions to use customer satisfaction-related information.

There are several questions surrounding the concept of attitude strength. One major issue focuses on whether it consists of ane single construct or several. Constructs used to define attitude strength vary from one study to another. They are aften highly correlated or even assumed to be completely interchangeable. Good reviews exist of explorative studies on attitude strength constructs, their correlation, and their interchangeability (e.g., Abelson 1988; Krosnick et al. 1993; Raden 1985). The main tenet is that several studies have moved away from considering attitude strength as one single construct toward operationalizing it into several underlying constructs (Abelson 1988; Krosnick et al. 1993; Lastovika \& Gardner 1979; Raden 1985).

For three different samples and five distinct issues, Pomerantz et al. (1995) found an identical factor structure indicating the existence of two dimensions underlying attitude strength. These factors were highly similar to factor solutions found in other studies (e.g., Abelson 1988; Lastovika \& Gardner 1979). The first factor is referred to as commitment and the second as embeddedness. Commitment can be described as the degree to which one feels secure and positive that one"s opinion concerning a certain entity is correct and one takes a strong position in defending that belief. Embeddedness, on the other hand, indicates how one is personally attached and involved with an issue, and the belief that one's attitude position reflects an inner self. walue. Given their success in predicting behavior and their resemblance with constructs found in earlier research, we confidently adopt embeddedness and commitment to conceptualize attitude strength.

Exploratory factor analyses revealed that attitude commitment was operationalized by items relating to attitude certainty as well as extremity of one's attitude position toward an issue (Pomerantz et al. 1995). Both characteristics are well established within the economic psychology and marketing literature (e.g. Davidson et al. 1985; Marks \& Kamins 1988; Van der Pligt et al. 1983). Certainty can be defined as "...the degree to which an individual is confident that his or her attitude toward an object is correct..." (Krosnick et al. 1993, p. 1132). Extremity relates to "... the extent to which an individual's attitude deviates from the midpoint of the favorable-unfavorable dimension..." (Krosnick et al. 1993, p. 1132).

Embeddedness was shown to be related to personal importance, ego involvement, and knowledgeability concerning some entity (Pomerantz et al. 1995). These 
characteristics also have a considerable foundation in academic literature (e.g., Jaccard \& Becker 1985; Krosnick 1988, 1990; Wood \& Kallgren 1988). Personal importance is defined as "..the extent to which an individual cares deeply about and is personally invested in an attitude..." (Krosnick et al. 1993, p. 1132). According to Pomerantz et al. (1995) ego involvement measures the centrality of one's attitude to the self-concept and value system. Finally, krosnick et al. (1993, p. 1133) refer to knowledgeability as

"...the amount of information about an object that accompanies one's attitude toward it in memory...". An overview of attitude strength and the constructs underlying it remains of little use for an empirical study on customer satisfaction-related information use without identifying relevant consequences. Therefore, the next section will elaborate on the cognitive and behavioral implications of attitude strength.

\subsection{Attitude Strength: Cognitive and Behavioral Consequences}

Various studies have shown that stronger attitudes contribute to (the likelihood of) behavior (e.g., Jaccard 1981; Jaccard \& Becker 1985; Pomerantz et all. 1995). This is exemplified by Krosnick and Petty $(1995$, p. 3) who state that "Attitudes can guide behavior, and strong attitudes should be more likely to do so than weak ones". Fishbein and Ajzen (1975) developed the attitude-behavior relationship with their theory of reasoned action. The theory proposes that actual behavior is determined by an intention to engage in that behavior. In turn, this intention is determined by a subject's attitude toward the behavior and environmental subjective norms concerning that behavior. As will become clear, we also adopt the basic ideas of the theory of reasoned action for the development of our conceptual framework. More specifically, it is expected that stronger attitudes concerning customer satisfaction will elicit intentions to engage in attitude-congruent behavior, which will be the selective use of satisfaction-related intelligence. The intentions will then function as predictors of actual behavior.

According to Pomerantz et al. (1995), with regards to the behavioral implications of attitude strength, a distinction must be made between mediating selective cognitive processes and resistance outcomes. As stated by Krosnick and Petty (1995, p. 3) "....attitudes can influence information processing and judgments". Therefore, attitude strength can directly influence (intentions of) behavior but can also have an indirect effect via selective cognitive processes, which in turn lead to resistance to change and attitude-incongruent positions (Eagly \& Chaiken, 1995). In attitude strength research, 
several of cognitive processes have been identified, such as selective exposure, selective attention, selective cognitive elaboration, selective perception, and selective memory (Eagly \& Chaiken 1993). In turn, Pomerantz et al. (1995) use selective judgment and attitude polarization measurements for assessing resistance outcomes and both issues have been the focus of numerous other studies as well (Eagly \& Chaiken 1993; Fiske \& Taylor 1991; Lord et al. 1979; Sherman et al. 1989).

The basic ideas of selective cognitive processes and resistance outcomes are important for understanding the effect of attitude strength on selective use of customer satisfaction-related information. The potential of attitude strength as a concept predicting selective use of satisfaction-related information emanates from these underlying processes. Besides in stronger intentions to act strong attitudes can be expected to result in more elaborate cognitive processes concerning the attitude entity. By thinking more elaborately about it one tends to selectively judge or evaluate information dealing with the entity on the basis of a normative framework that has been developed concerning the entity. Lee et al. (1987) refer to this phenomenon as 'belief perseverance', which they base their finding on that research confirming prior beliefs tends to be rated higher and is more likely to be used. On the basis of the previous discussion, we now will develop a conceptual framework on the effects of attitude strength concerning customer satisfaction on marketing decision-maker intentions to selectively use satisfaction-related information.

\subsection{Conceptual Framework and Research Hypotheses}

Based on Pomerantz et al. (1995), we consider attitude strength to be a twodimensional concept encompassing commitment and embeddedness. Our objective is to empirically test the relationship between decision-maker attitude stirength concerning the importance of customer satisfaction and intentions to selectively use customer satisfaction-related marketing intelligence. On the basis of the previous literature review, we expect this relationship to be direct but allso to be mediated by other factors.

Following from its general conceptualization, attitude commitment refers to feeling secure and positive that one"s attitude concerning the importance of customer satisfaction is correct and to taking a strong position in defending this belief. We expect that commitment will increase the level to which one cognitively elaborates upon issues relating to customer satisfaction, for example on the positive consequences of satisfying customers for an organization. For important decisions 
individuals tend to reflect more on the matter (Jaccard et al. 1995). Furthermore, several strength variables were found to increase selective cognitive elaboration (Chaiken et al. 1995; Chaiken \& Vates 1985). More specifically, Pomerantz et al. (1995) found empirical evidence for a positive association between attitude commitment toward an issue and selective cognitive elaboration.

$H_{1}$ : There will be a positive relationship between commitment toward the importance of customer satisfaction and cognitive elaboration on customer satisfaction.

In the literature there is little research explicitly examining the relationship between attitude strength and selective judgment (Chaiken et al. 1995; Houston \& Fazio 1989; Miller et al. 1993). However, Pomerantz et al. (1995) concluded that the level of commitment toward an issue had a positive effect on selective judgment of issue-related information. Therefore, we expect that decision-maker commitment toward customer satisfaction will contribute to the selective judgment of satisfactionrelated information. This judgment is based on an attitude-congruent normative framework used to evaluate the information.

$H_{2}$ : There will be a positive relationship between commitment toward the importance of customer satisfaction and selective judgment of customer satisfaction-related information.

Next, decision-makers committed to customer satisfaction are likely to show higher intentions to act in accordance with this attitude. More specifically, they will display higher intentions to use information, supporting their attitude that customer satisfaction is important for the organization. As mentioned, the positive attitudebehavior reliationship has been supported by various other studies (e.g., Jaccard 1981; Jaccard \&. Becker 1985; Pomerantz et al. 1995). Pomerantz et al. (1995) showed that attitude commitment positively influences intentions to act in accordance with this attitude.

$H_{3:}$ There will be a positive relationship between commitment toward the importance of customer satisfaction and intentions to selectively use customer satisfactionrelated information.

The effects of attitude embeddedness on cognitive elaboration have been well documented in psychological literature as well as in marketing settings. Petty and Cacioppo (1979) showed that issue involvement and personal relevance (similar to 
what Pomerantz et al. (1995) refer to as ego inwolvement and personal importance) increase one's motivation to process information. Since then the relationship between issue involvement and processing effort has received considerable attention and been confirmed in various other studies as well (e.g., Beatty \& 5 mith 1987; Chaiken 1980; Greenwald \& Leavitt 1984; Maheswaran \& Myers-Levy 1990; Petty \& Cacioppo 1986a, 1986b; Petty et al. 1983). Furthermore, prior knowledge has also been shown to positively influence information processing (Maheswaran \& Sternthal 1990). This leads us to expect that embeddedness of the importance of customer satisfaction will increase decision-maker cognitive elaboration on customer satisfaction.

$H_{3}$ : There will be a positive relationship between embeddedness of the importance of customer satisfaction and cognitive elaboration on customer satisfaction.

Similar to attitude commitment, we expect that embeddedness will also contribute to decision-maker selective judgment. Decision-makers in whom the importance of customer satisfaction is strongly embedded are more likely to selectively judge customer satisfaction-related information against a normative framework.

$H_{5}$ : There will be a positive relationship between embeddedness of the importance of customer satisfaction and selective judgment of customer satisfaction-related information.

In decision-making experiments it has been shown that prior knowledge, underlying embeddedness, influences behavior of information acquisition and use (Lee et al. 1999). Following Pomerantz et al. (1995) we posit that embeddedness of the importance of customer satisfaction will directly result in higher intentions of selectively using satisfaction-related information. Information that is in accordance with this attitude is more likely to be used for decision making purposes than information that does not conform to this attitude (e.g., information not underlining the importance of satisfying customers).

$H_{6}$ : There will be a positive relationship between embeddedness of the importance of customer sotisfaction and intentions to selectively use customer satisfactionrelated information.

Selective cognitive elaboration on a specific issue strongly increases the level of selective judgment of information concerning this issue (Pomerantz et al. 1995). As a consequence, we expect that decision makers who cognitively elaborate on customer 
satisfaction and its importance for the organization (who feel they know what they are talking about and dealing with') are more likely to judge/evaluate customer satisfaction-related information on the basis of an attitude-congruent normative framework. The respective hypothesis is as follows:

$H_{7}$ There will be a positive relationship between cognitive elaboration on customer satisfaction and selective judgment of customer satisfaction-pelated information.

Finally, decision-makers who selectively judge customer satisfaction-related information on the basis of their personal attitude or belief are likely to act in accordance with this belief. Based on Hogarth and Makridakis (1988), we expect that they are more likely to express intentions of using information that underlines the importance of satisfying customers for the success of organizational activities:

$H_{g}$ : There will be a positive relationship between selective judgment of customer satisfaction-related information and intentions to selectively use customer satisfaction-related information.

The hypotheses we formulated above can be summarized by the conceptual model presented in figure 5-1.

\section{Figure 5-1: Conceptual Model of Attitude Strength}

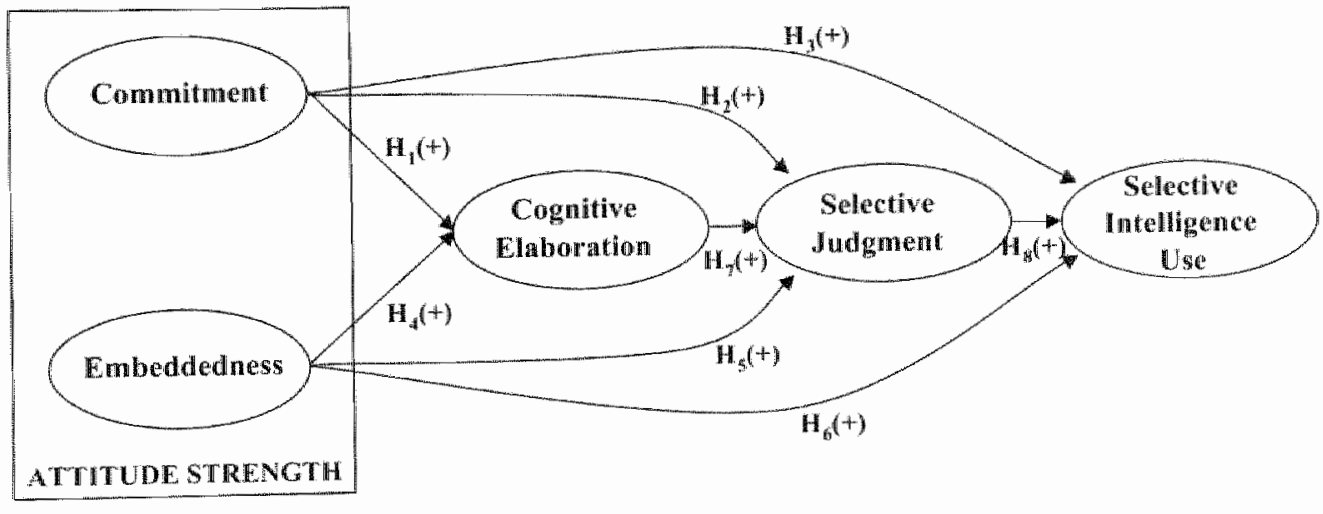

The next section will elaborate on an empirical study conducted to test our theoretical framework on customer satisfaction attitude strength. 


\subsection{An Empirical Study}

\subsubsection{Research Design and Data Collection}

The empirical study for testing our hypotheses was conducted among decision-makers of a multinational office equipment manufactuper. A service research center in the Netherlands has been conducting a large-scale international customer satisfaction survey in several countries focusing on after-sales services, such as product delivery, installation, customer support, service visits, and complaint handling. Respondents were required to be in marketing decision-making positions in which they would come into contact with the customer satisfaction-related information produced by the research project. Respondents were selected on the basis of their job titles for which descriptions were made available by the organization's management. Job titles of actual respondents included business analysis positions (6\%; e.g., business analyst), strategic decision-making positions (10\%; e.g., senior marketing executive), productrelated positions (17\%; e.g., product manager), and service-related positions (52\%; e.g., service manager).

Questionnaires were distributed personally by thoroughly instructed students who were active as interns in the organization. This procedure allowed us to exercise a certain level of control over the composition of the sample in terms of validity and the final response rate. In total, 174 questionnaires were handed out. Ultimately, the response totaled 121 respondents meeting the required criteria. This represents a response rate of $70 \%$, which is likely to be that high due to the personal approach in the sampling procedure.

\subsubsection{Questionnaire Development}

The questionnaire was developed by rewiewing the measurement instruments of attitude strength and rellated constructs in previous research. The items were adapted to the current marketing decision-making situation focusing on customer satisfaction and its importance to the organization. The questionnaire was extensively pre-tested and refined prior to actual data collection by administering it to several subjects from the target population who in in-depth interviews commented on potential misunderstandings and misconceptions. In order to avoid test-retest biases subjects involved in the pre-testing of the questionnaire were not included in the final sample. On the basis of the pre-test several items were adapted before including them in the final questionnaire. The questionnaire was of a structured undisguised format. 
Since the main focus was on determining a decision-maker"s predisposition toward customer orientation, an issue that might be considered a socially desirable business value, complete anonymity was explicitly stressed. This would increase the likelihood of ewoking true attitudes toward customer satisfaction and its importance. All items in the questionnaire were stated in a 9-point Likert-type format ranging from (1) totally disagree to (9) totally agree.

The items measuring the commitment and embeddedness constructs were based on the study by Pomerantz et al. (1995). Decision-maker commitment toward the importance of customer satisfaction was measured by four items and three items were used to operationalize the level of embeddedness of this issue. The respondent initiated free response procedure used by Pomerantz et al. (1995) to assess cognitive elaboration could not be used in our survey-based study. Therefore, the four items measuring cognitive elaboration of decision-makers about customer satisfaction were developed specifically for this study. However, the basic ideas of Pomerantz et al. (1995) were left intact. The same holds for the three items operationalizing selective judgment, which also had a strong foundation in Pomerantz et al.'s (1995) study.

Finally, decision-maker intentions to selectively use customer satisfaction-related intelligence were operationalized by four items based on a study on market intelligence use by Maltz and Kohli (1996). Table 5-1 presents sample items for each of the constructs.

\subsubsection{Construct Validation}

Prior to testing the hypotheses we assessed the construct validity of the constructs used in our empirical research. We used structural equation modeling, more in particular confirmatory factor analysis, in LISREL 8 (Joreskog \& Sörbom 1993) to evaluate unidimensionality, reliability, convergent validity and discriminant validity (Anderson \& Gerbing 1988; Bagozzi 1980; Bollen 1989; Gerbing \& Anderson 1988; Kumar \& Dillon 1987; Steenkamp \& van Trijp 1991).

The proposed measurement model with five constructs and eighteen indicators showed an adequate fit to the data: $\chi^{2}(125)=179.76(p<0.001), \operatorname{RMSEA}=0.048, G F=0.87$. AGFI $=0.82$, $T L I=0.95, C F I=0.96$ (Bagozzi \& Yi 1988; Bentler 1990; Bollen 1989; Hoelter 1983; Marsh \& Hocevar 1985; Steenkamp \& van Trijp 1991). However, after inspection of the t-values, the pattern of standardized residuals and the modification indices one item for selective intelligence use was deleted (Anderson \& Gerbing 1988; 5teenkamp \& van Trijp 1991). Subsequently, we obtained an even better fit: $\chi^{2}(109)=130.31$ $(\mathrm{p}=0.08), \mathrm{RMSEA}=0.032, G \mathrm{FI}=0.89, \mathrm{AGF}=0.85, \mathrm{TLI}=0.98, \mathrm{CF}=0.98$. Among these the Tucker-Lewis Index (TLI) (Bentler \& Bonett 1980; Tucker \& Lewis 1973) and the 
Comparative Fit Index (CF) (Bentler 1990) seem to be relatively unaffected by sample size and model complexity (Marsh et al. 1988, 1996). The Root Mean Square Error of Approximation (RMSEA) might provide even a better indication of goodness-of-fit (Steiger 1990). The walue of the RMSEA equals 0.032 and is well bellow the recommended cut-off value of 0.08 .

\section{Table 5-1: Sample Items}

\begin{tabular}{|c|c|}
\hline Coustruet & Sample Item ( $1=$ Strongly Disagree, $9=$ Sirongly Agree) \\
\hline Commüment & 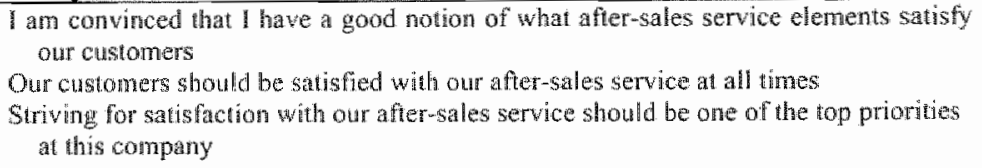 \\
\hline Entheddedness & 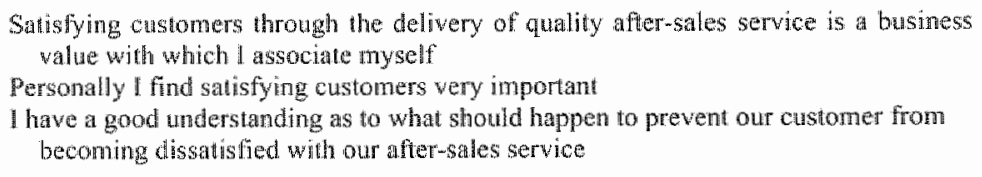 \\
\hline $\begin{array}{l}\text { Cognitive } \\
\text { Elaboration }\end{array}$ & 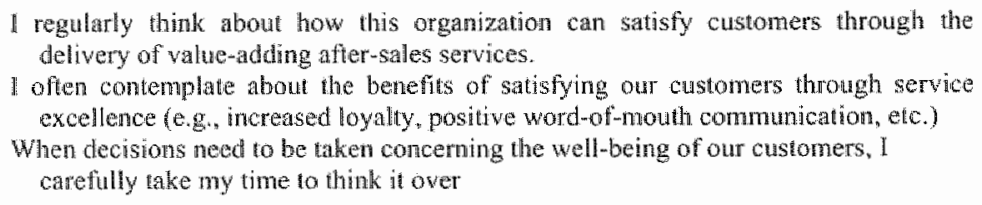 \\
\hline $\begin{array}{l}\text { Selective } \\
\text { Judgmeni }\end{array}$ & $\begin{array}{l}\text { Well-conducted satisfacion research should contain detailed information on critical } \\
\text { after-sales service improvement areas } \\
\text { A convincing after-sales service survey should highlight customer complainis } \\
\text { A convincing atter-sales service survey should cover all elements of the service } \\
\text { delivery process }\end{array}$ \\
\hline 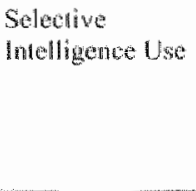 & 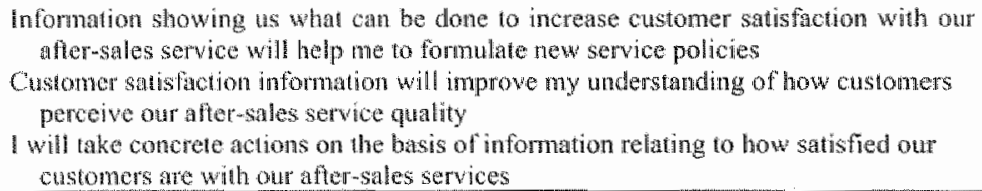 \\
\hline
\end{tabular}

Within method-convergent validity was assessed testing the significance and magnitude of the elements of the matrix $\Lambda^{*}$. All indicator loadings were significant at $\alpha=0.05$ and $t$-values ranged from 5.43 to 13.48 . The standardized indicator loadings all exceeded 0.50 ; the average indicator loading is 0.76 for the exogenous constructs (pertaining to commitment and embeddedness) and 0.82 for the endogenous constructs (pertaining to cognitive elaboration, sellective judgment, and selective intelligence use). Discriminant validity was evaluated by testing whether pairs of constructs were correlated less than unity. We used a $\Delta x^{2}$ with one degree of freedom to test for unity between the constructs. All tests were significant at $\alpha=0.05$. Moreover, 
the reliability of the constructs in the model was evaluated using composite reliability and variance extracted measures (Fornell \& Larcker 1981; Jöreskog 1971b).

The composite reliability scores ranged from 0.78 to 0.92 . The Cronbach's $\alpha$ reliability coefficients along with construct means, standard deviations, and intercorrelations are presented in table 5-2. The reliability scores all exceed the threshold value of 0.70 as proposed by Nunnally and Bernstein (1994). After assessing the construct validity we proceed with testing the hypotheses in our hypothesized model.

Table 5-2: Means, standard Deviations, Intercorrelations, and Coefficients $\alpha^{\text {al }}$

\begin{tabular}{|c|c|c|c|c|c|c|c|c|}
\hline Nr. & Cronsitruet & $\mathrm{M}$ & $\mathrm{SD}$ & 1. & 2. & 3. & 4. & 5 \\
\hline 1. & COMM & 5.56 & 1.45 & 0.77 & & & & \\
\hline 2. & FMB & 6.93 & 1.70 & 0.03 & 0.86 & & & \\
\hline 3. & COGNEL & 5.94 & 1.51 & $0.61^{\circ *}$ & $0.24^{* 0}$ & 0.94 & & \\
\hline 4. & SELUUDG & 5.66 & 1.79 & $0.38^{* 4}$ & $0.21^{5+4}$ & $0.611^{*-*}$ & 0.81 & \\
\hline 5. & INTUSE & 4.07 & 1.95 & $0.51^{\prime \prime *}$ & 0.13 & $0.45^{-6 t}$ & $0.38^{* i x}$ & 0.89 \\
\hline
\end{tabular}

Cronbuch's o rellability coefficients on diagonal

3 COMM=Comminment; EMB=Embeddedness; COGNEL=Cognitive Enaboration; SELUDGmSelectwe Judgment; NTUSE-Selective lonelligence Use

* Significant iat $\alpha=0.05(1-$ ated $)$

** Significant at $\alpha=0.01$ (1-kxted)

\subsubsection{Hypotheses Testing}

The hypothesized model in figure 5-1 was tested by estimating the structural model. With the variance-covariance matrix of the indicator variables as imput the parameters in the latent variable model were estimated using maximum likelihood estimation (Bentler \& Chou 1987; Bollen 1989; Hayduk 1987; Loehlin 1998). The model shows a satisfactory fit to the data: $\chi^{2}(110)=130.46 \quad(p=0.089)$, RMSEA=0.032, GFH=0.89, $A G F \mid=0.85, T L I=0.98, C F I=0.98$. Determination of rejection (or failure to reject) is based on the path coefficients ( $B$ and $\Gamma$ ) and their supporting statistics (see table 5-3). The results of the path analysis are also visualized in figure 5-2.

As can be concluded from table 5-3 and figure 5-2, we fail to reject hypotheses 1, 3. 4,7 , and 8 at a $5 \%$ significance level. Hypotheses 2, 5, and 6 , however, are rejected: none of the respective path coefficients turned out to be significant. More specifically, we found that decision-maker commitment toward customer satisfaction has a relatively strong significant positive effect $\left(\gamma_{1,1}=0.70\right)$ on cognitive elaboration on issues concerning customer satisfaction, such as its consequences for the organization. In addition, a direct positive relationship $\left(\gamma_{1,3}=0.44\right)$ between commitment and intentions to selectively use customer satisfaction-related information for making decisions was found: decision-makers highly committed toward satisfying customers are more likely to use attitude-congruent information that, for example, indicates what an 
organization can do to increase its customers" satisfaction level. However, no significant effect was found for commitment on the selective judgment of customer satisfaction-related information.

Table 5-3: Estimated Path Coefficients

\begin{tabular}{|c|c|c|c|c|}
\hline Relationships & Hypothesis & $\begin{array}{l}\text { Unstandardized } \\
\text { Path Coefficient }\end{array}$ & $\begin{array}{c}\text { Standardized Path } \\
\text { Coefficient }\end{array}$ & Hypothesis Testing \\
\hline COMM $\rightarrow$ COONEL & $\mathrm{H}_{1}$ & $0.63(6.14)$ & 0.70 & Falled to rejeci \\
\hline COMM $\rightarrow$ SELIUDO & $\mathrm{H}_{2}$ & n.:s. & m.s. & Rejected \\
\hline COMM $\rightarrow$ NNTUSE & $H_{3}$ & $0.67(3.86)$ & 0.44 & Failed to reject \\
\hline $\mathbb{E M B} \rightarrow$ COGNEL & $\mathrm{H}_{4}$ & $0.17(2.34)$ & 0.18 & Failed to reject \\
\hline EMB $\rightarrow$ SELUUDG & $\mathrm{H}_{5}$ & n.s. & $\mathrm{n}, \mathrm{s}$ & Rejecied \\
\hline EMB $\rightarrow$ NTTUSE & $\mathrm{H}_{16}$ & n.s. & n... & Rejerted \\
\hline COGNEL $\rightarrow$ SELJUDG & $\mathrm{H}_{7}$ & $0.79(4.65)$ & 0.66 & Falled to reject \\
\hline SELUUDG $\rightarrow$ INTUSE & $\mathrm{H}_{3}$ & $0.43(2.74)$ & 0.30 & Failed to reject \\
\hline \multicolumn{5}{|c|}{$R^{2}$ COGNEL $=0.53 \cdot R^{2} S E L J U D G=0.49 ; R^{2}$ INTUSE $=19.42$} \\
\hline $\begin{array}{l}\text { COMM }=\text { Comminte } \\
\text { Judgment; INTUSE } \\
\text { Corresponding l-walt }\end{array}$ & between pa & $\begin{array}{l}\text { Tess: COGNEL } \\
\text { Use } \\
\text { es }\end{array}$ & Elaboration: & SELIUDG = Selective \\
\hline
\end{tabular}

\section{Figure 5-2: Causal Model of Attitude Strength ${ }^{\text {a,b }}$}

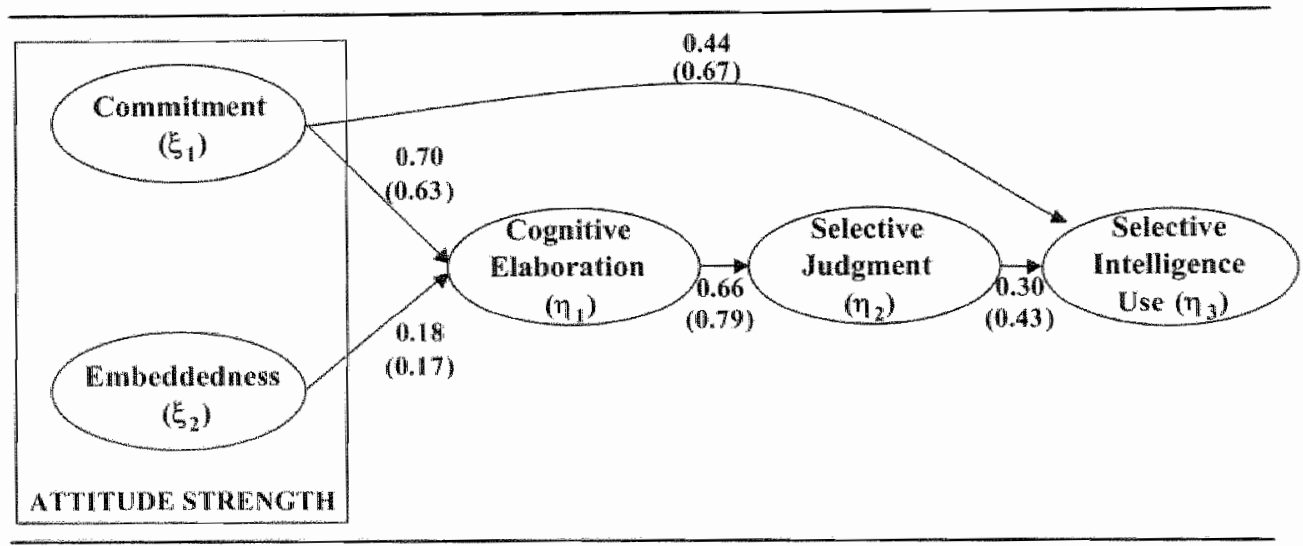

"Dnly significant path coefficients are shown $(a=0.05)$

b Unandardizad path coefficients between parentheses

For embeddedness of customer satisfaction, the second dimension of attitude strength, we also found a significant positive effect $\left(\gamma_{2,3}=0.18\right)$ on cognitive elaboration on issues concerning customer satisfaction. No relationships were found between attitude embeddedness and selective judgment or intentions to selectively use customer satisfaction-related information. This seems to indicate that embeddedness. of customer satisfaction, involving a deeper linkage of the attitude to one's selfconcept, value system, and knowledge structure (Pomerantz et al. 1995), indirectly 
influences decision-maker selective judgment and intentions to act on this attitude through evoking cognitive processes on this matter. On the other hand, commitment toward the importance of satisfying customers directly triggers intentions to selectively use customer satisfaction-related information.

in correspondence with Pomerantz et al. (1995) we also found a strong positive effect $\left(\beta_{1,2}=0.66\right)$ of cognitive elaboration on decision-maker selective judgment. In other words, the effect of commitment toward customer satisfaction on selective judgment of satisfaction-related information is mediated by the level to which one cognitively elaborates on customer satisfaction. Furthermore, it can also be concluded that higher selective judgment of information on customer satisfaction directly contributes to a decision-maker's intention to act in accordance with the attitude and the normative framework used to evaluate the information on $\left(\beta_{2,3}=0.30\right)$ : he or she will be more inclined to use information that underlines the importance of customer satisfaction for the organization.

\subsection{Conclusion}

\subsubsection{Discussion}

Although not all hypothesized relationships were confirmed, our study indicates that attitude strength is a relevant concept in explaining information use. The results enable the development of a more profound understanding of managerial decisionmaking processes concerning customer satisfaction and marketing operations in general. The dimensions of attitude strength, commitment and embeddedness, provide key insights into subjective factors creating a bias or non-bias toward customer satisfaction-related information. They explain how a personal prejudice against or favoritism for this issue can lead to the acceptance or rejection of intelligence.

The concept of attitude strength and the results of this study offer an interesting complementary view to previous research, which mainly focused on how instrumental issues, such as the quality of research contents or the presentation of research results influence marketing intelligence use. With this study it becomes clear that these variables represent only one flipside of the coin. Acceptance and use of information is also determined by subjective biases or non-biases following from the strength with which attitudes are being held. Subjectivity can have great influence, no matter how good the quality of the research being conducted or the intelligence provided. 
Our results show a sequential flow from the attitude strength commitment dimension to intentions of selectively using customer satisfaction-related information. Commitment, focusing on the certainty that one's opinion concerning the importance of customer satisfaction is correct and the extremity of taking a strong position in defending that bellef, increases cognitive thinking processes on customer satisfactionrelated issues. This might for example be on the positive effects that satisfying customers has for the organization, such as higher sales, higher customer loyalty, and positive word-of-mouth communications. The positive effect of cognitive elaboration on selective judgment indicates that by thinking about customer satisfaction the issue is placed in a cognitive context, to be translated into a normative framework used to judge satisfaction-related information by. Such framework will contain attitudecongruent requirements. This could be that information should underline the importance of satisfying customers by stressing its effect on customer loyalty. In turn, the results indicate that decision-makers who selectively judge customer satisfactionrelated information show higher intentions to selectively use information that "fits" into one's framework. Information for example demonstrating that efforts undertaken to increase customer satisfaction levels indeed lead to a pay-off in terms of extra sales is more likely to be used for decision-making purposes.

In addition to this sequentiall process, the relevance of commitment toward the importance of customer satisfaction is exemplified by its direct effect on selective intelligence utilization. This seems to indicate that persons who take on a strong standpoint on customer satisfaction and its importance will be more inclined to act accordingly and use information supporting their standpoint. Overall, therefore, it can be concluded that customer satisfaction commitment has a positive impact on the selective acceptance and use of customer satisfaction-related information.

Customer satisfaction embeddedness, referring to one's personal attachment and involvement with customer satisfaction and the belief that the position concerning this issue is reflecting some inner values or concepts, was found to increase cognitive elaboration on customer satisfaction. A decision-maker's involvement with customer satisfaction, the personal importance being attached to it, and prior knowledge of customer satisfaction will heighten the level to which one thinks about satisfying customers and what it can do for one's organization. An underlying explanation for the insignificant relationship with selective judgment and intelligence use could be sought in the deeper, internally directed nature of embeddedness. In contrast to attitude commitment, represented as a strong, externally oriented standpoint held by a decision-maker, deeper personal attachment with customer satisfaction is apparently not likely to evoke intentions to act. It first seems to be transferred to a higher level 
through cognitively thinking about it and these cognitions are then used to develop a normative framework to judge satisfaction-related information by.

\subsubsection{Theoretical Implications}

Since the present study is a preliminary attempt to investigate attitude strength in a marketing decision-making context several theoretical implications need to be taken into account. Acknowledging these implications will be beneficial to marketing intelligence utilization research, but also to research aimed at other marketing-related fields that could benefit from such psychological perspective. An example could be marketing innovation management, in which success rates might depend heavily on the attitudes of the persons involved, e.g., customers and decision-makers.

First, attitude strength research conventionally focuses on quite controversial issues, such as capital punishment, legalized abortion, and environmental preservation (e.g. Pomerantz et al. 1995). Customer satisfaction might not be perceived as particularly controversial. It could even be nominated as a socially desirable value among business practitioners. Consequently, one's attitude toward customer satisfaction and its importance to an organization is not likely to elicit such extreme bipolar reactions as the more social significant and controversiall issues are likely to do. As such, a positive-directed tendency in the data might be present. Therefore "future research should also focus on business-related issues likely to elicit more extreme attitudes in both directions, such as the allocation of (marketing) budgets. Decisions then might have a quite varied impact on the parties involved.

Second, customer satisfaction can be interpreted in various ways by different respondents. Future research on the relationship between attitude strength and marketing intelligence use could focus on issues that are more concise. It might for example be interesting to study effects of decision-maker attitudes toward concrete actions that an organization can undertake to increase customer satisfaction levels, such as improving the product offering or increasing service levels. This might lead to more univocal research results, which could further substantiate our conclusions.

Third, as mentioned before, decision-maker attitude strength is only one of many factors that have an impact on the utillization of marketing intelligence. Attitude strength irepresents a subjective characteristic of information receivers. However, prior research has already proven the impact of instrumental issues of the research project itself (e.g., quality of contents, quality of form, actionability and acceptability of results) on market research intelligence use (Deshpandé \& Zaltman 1982, 1984). We feel that attitude strength should not be seen as a substitute to the explanations offered by these studies; it rather facilitates and complements them. It offers a better 
understanding of the psychological processes that play a role in managerial decisionmaking. Therefore, future research should combine the concept of attitude strength with instrumental variables. In doing so, determinants of marketing intelligence use can be studied simultaneously from different perspectives and additional explained variance can be assessed. In addition, the model could also be extended at the frontend by including drivers of attitude strength, for example the level of decision-maker experience as suggested by Perkins and Rao (1990).

Next, even though all respondents were working for the same organization and face the same business environment, some variance in the data could be due to situational forces. For example, the functional backgrounds of respondents were varied and future research could investigate whether and, if so, how such functional differences have an impact on attitudes. Another suggestion for future research would be to replicate the study with decision-makers of organizations active in other industries in order to assess generalizability of results.

A final suggestion for future research relates to the research design that we used to study the effects of attitude strength. In this study, a cross-sectional study was conducted by administering questionnaires to decision-makers. The questionnaire contained Likert-type items, all focusing on the importance of satisfying customers, on which respondents could react by self-reporting their level of (dis) agreement with a particular statement. Traditional research on attitude strength, however, is often of a more experimental nature. Subjects then are provided with issue attitude-congruent and -incongruent materials on which they have to react (cf. Pomerantz et al. 1995). Based on these reactions indexes are calculated expressing a tendency to selectively think about the issue or judge information on it. The main advantage of this procedure is that automatic selectivity is assessed more explicitly and according to real-life in which decision-makers are often confronted with information covering 'both sides of the story'. We suggest that future research efforts should adopt such an experimental approach in order to validate the conclusions we draw from our cross-sectional study. This could be done by simultameously confronting respondents with information stressing the importance of satisfying customers and with information giving counterarguments concerning this issue.

\subsubsection{Managerial implications}

Our results have several interesting managerial implications, both for customer satisfaction or other marketing-related intelligence providers (e.g., marketing research agencies) as well as organizational management. Since attitudes determine acceptance of research information, insight into decision-maker attitudes is important, 
especially when a study deals with a topic crucial for the organization. Researchers and managers need to recognize that the strength with which attitudes are being held has an impact on cognitive processes, which in turn determine the evaluation and use of is sue-related information.

Both attitude commitment and embeddedness play a significant role in the acceptance and use of customer satisfaction-related information. Therefore, decisionmaker attitudes should be strengthened by focusing on these dimensions. Researchers conducting customer satisfaction surveys and management should make decisionmakers more aware of the importance of satisfying customers for the organization and their own well-being and get them more involved and committed toward the issue. Furthermore, increasing knowledge levels on the benefits of satisfying customers could be achieved by organizing training sesslons or publishing periodicals or research reports in which attention is paid to this issue. This will improve the acceptance and use of information obtained by conducting expensive satisfaction surveys.

The results also offer insight into resistance processes against organizational and structural change. Often changes are proposed on the basis of marketing research results that not only affect the position or quality of product and service offerings, but also have major secondary consequences. Strategic changes often require substantive organizational adjustments. To facilitate this transition process senior managers could focus on the strength with which attitudes are being held by organizational members. For example, the outcome of a customer satisfaction survey could be that the organization is too product-focused and that the organization lacks customer orientation. For striving for $100 \%$ customer satisfaction massive organizational change at the cultural level is likely to be required. Improving attitudes toward customer satisfaction is likely to lower resistance against measures that need to be taken.

Company-wide campaigns could be run to stress the importance of satisfying customers and what the effects will be on the bottom line. Knowledge on the importance for long-term survival and involvement with the is sue should be raised, leading to stronger embeddedness of customer orientation into the company. Increasing decision-makers commitment will also facilitate cognitive processes, the selective judgment of satisfaction-related information, and acceptance of information concerning customer satisfaction. 

Chapter 6

Conclusion 


\section{Conclusion}

\subsection{Synopsis}

Our mission throughout this thesis was to develop a deeper understanding of marketing inteligence effectiveness in international services marketing. Our main criterion for conceptualizing effectiveness was whether or not international marketing intelligence is likely to be used by marketing as well as other decision-makers within a service firm. To accomplish our mission, we introduced the metaphor of the intelligence service encounter in which specific attention is paid to intelligence attributes, as well as the characteristics of the acting parties, namely intelligence providers and intelligence users. In the introduction, we formulated the main research question of this thesis as follows:

Which factors contribute to the effectiveness of marketing research intelligence in international service organizations?

Four related research questions have been developed to specify our main research question:

1) How can cross-cultural variation be taken into account in international services research?

2) How do additional information attributes contribute to effectiveness of international service intelligence?

3) What researcher characteristics contribute to effectiveness of international service intelligence?

4) What user characteristics contribute to effectiveness of international service intelligence?

These research questions were based on a multifaceted research approach, consisting of three distinct perspectives, that we adopted to focus our vision. First, the intelligence attribute perspective pertained to characteristics of international service intelligence and how these relate to information utilization by decision-makers. The basic premise is that, in order to be used effectively by marketing and other decisionmakers in international service firms, international intelligence needs to have certain properties. Next, the relational exchange perspective on intelligence effectiveness 
originated from the services research domain and conceptualized the provision of international service intelligence as a service encounter involving a relationship between researcher and user. Our final perspective, referred to as the attitudinal perspective, focused on the impact of user attitudes on cognition and behavior with respect to international service intelligence.

Four specific research projects have been presented in order to address the aforementioned research questions. Each research project incorporated both a conceptual development and empirical research components. First, we demonstrated how variation on cross-cultural characteristics could be taken into account when generating intelligence on international service performance. Second, as an extension of the intelligence attribute perspective, we examined the impact of informational presence or absence on decision-maker preference for and utilization of international service intelligence. A differentiation was made between two types of information: information (I) on cross-cultural comparability of intelligence and (11) on financial accountability of tactical actions to be taken on the basis of the intelligence. Next, the intelligence attribute perspective was supplemented with the relational exchange perspective by also studying the effects of perceived researcher interpersonal characteristics on service intelligence effectiveness. Finally, our perspectivism approach focused on attitudes of intelligence users and how these have an effect on cognition and utilization behavior with respect to international service intelligence. By adopting the attitudinal perspective, our multifaceted research cycle was completed.

The remainder of this final chapter is structured as follows. In section 6.2, we attem to tevelop a multifaceted vision on effective international service intelligence, which goes beyond the conclusions that were already drawn on the basis of the individual research projects. This will serve as an integrative answer to our main and specific research questions, as formulated above. The chapter concludes with a perspective on future research.

\subsection{A Multifaceted Vision}

Our vision on the effectiveness of international service intelligence is strongly embedded in the fact that, due to emerging technological developments, the delivery of international services can be incrementally characterized by a multifaceted nature. Advances in information and communication technology, such as the internet, intranet, extranet, hand-held mobile communication devices, etc., enable service providers to enter new service playgrounds and reach customers through new, 
innovative service delivery modes. Technology in service delivery facilitates the broadening of business horizons, both with respect to service content as well as to service location. In this thesis, several examples were given of service providers that, through the infusion of technology in their service processes, are able to enter new international markets and serve international customers. Besides the well-known advantages of internationalization (e.g., market expansion, avoidance of market saturation, acquisition of know-how through new business partners, etc.), a significant consequence of these developments is that service firms face the challenge of addressing issues that lie beyond their home turf. In our modern age of service delivery, it has been frequently argued that particularly cross-cultural variation between countries thas a not to be ignored impact on service customers, their perceptions of service performance, and their behavior in the international services setting. As such, technology-infusion in services does not appear to be culturally neutrall, despite the fact that this is an ephemera often associated with cyberspace. As is the case for traditional service settings, the measurement of performance of innowative, technology-infused services is an essentiall step in moving towards service success. Intelligence on how customers perceive service quality will help service firms to develop better service management policies, irrespective of the service nature. Due to higher levels of uncertainty and complexity that are commonly associated with services, as well as the consequences of cross-cultural diversity in international service markets, it there would seem to be an urgent need for the development of a methodology and research instruments that enable a differentiation and refinement of the interaction between technology-infusion in services and cross-cultural background. Such techniques can be used for the generation of more effective international service intelligence. As was demonstrated in this thesis, the use of multilevel modeling holds a promising potentiall for nuancing the impact of culture's consequences in servilce performance intelligence.

Our finding that cultural differences indeed appear to be influential on service performance evaluations when the technological factor is more prominent in service delivery should be taken into consideration when interpreting intelligence on perceived service performance and developing service management policies. Intelligence that is used for decision-making in international services needs to be reconsidered beyond its multi-cultural context. For it may very well be that crosscultural variation rather than business or employee performance accounts for differences in conclusions of international service intelligence. Transferring established ideas, e.g." providing quality services to customers will result in higher customer satisfaction, irrespective of service setting or geographical location, may be dangerous. In some countries, one specific type of service interaction may be more 
effective than in other countries, due their specific cultural background. More specifically, a "think global, act local" strategy, often used in the traditional face-to face service approach, might also be effective for technology-infused services. In light of the technological developments in the services arena, the approach outlined in this thesis for taking cross-cultural variation in international services research into account enables both intelligence providers and users to explicitly take the richness of new media for service delivery and interactive service processes into account. A focus on additional cues facilitates the development of further insight into how service satisfaction can be resolved into service performance and how concrete measures could and should be taken to improve service performance. Consequently, service intelligence will become more effective in a (media) richer international service intelligence setting.

A double-edged sword is unsheathed with respect to the issue of uncertainty in services. It has been argued that the arena of international services can be characterized by higher levels of uncertainty. In addition, the fact that service intelligence hardly ever is complete increases decision-making uncertainty and results in an uncertainty multiplier effect. In order to effectively deal with uncertainty in international services through effective intelligence, it is important to understand what the effects will be of presence or absence of international service information. This is substantiated by our finding that information users are not likely to ignore missing information, but tend follow a strategy of inferential, assumption-based reasoning: other available information will be used to fill in an intelligence gap and reduce decision-making uncertainty. More specifically, two types of information seem to be relevant in an international services setting. Our plea for the necessity of taking cross-cultural variation in services into account calls for the inclusion of additional information on cross-culturall issues and how these affect service performance as well as performance intelligence. Furthermore, due to the substantial investments associated with the internationalization of services information on financial accountability of service investments will be desirablle as well. Given these informational types, it was concluded that decision-makers value intelligence that is as. complete as possible. However, they also tend to differentiate between information on the basis of its perceived decision-making relevance. Information on financial consequences of international service investments seems to be preferred over crosscultural info. On the other hand, intelligence seems to be evaluated even more positively when it includes both cross-cultural information and financial accountability information.

Since international service intelligence can be considered the outcome of the interaction between intelligence providers and users, we conceptualized it as 
representing the technical quality dimension of the researcher-user service encounter. A multifaceted approach seems to be useful as well for conceptualizing this dimension. In addition to attributes, such as paying attention to cross-cultural variance and the financial consequences of service investments, two additional informational properties seem to be quintessential for improwing the effectiveness of international service intelligence. First, our conclusions suggest that content quality is a key driver of user satisfaction evaluations and, indirectly, of intelligence utilization. Researchers can improve users. perception of content quality by making information as well as research procedures as transparent as possible to information users and, in doing so, create a sense of value for money. Second, intermational service intelligence will be more effective when decision-makers perceive the informational content as matching with the service organization's political context. This requires a deeper understanding of the user decision-making situation, the beliefs being held, and the resources availlable.

In essence, the intelligence service encounter involves a relationship between researchers and intelligence users. Previous studies on relationship marketing, services marketing, and marketing intelligence utilization suggest that interpersonal factors might play a role in the establishment of effective international service intelligence. Our findings also provided clear support for relational dimensions of international service intelligence and lead to a more comprehensive understanding of the dynamics that operate in the researcher-user relationship over time. In the starting phase, international service intelligence effectiveness seems to be determined most strongly by user satisfaction and political acceptability. When the relationship starts to develop over time, however relational factors strongly emerge as crucial determinants of international service intelligence effectiveness. Therefore, intelligence providers need to transcend the short-term perspective and move towards an account-management approach in order to establish effective relationships with their clients, i.e. users. In this respect, researcher interpersonal characteristics, such as integrity, flexibility, service-oriented, and objectivity will play a quintessential role. Adapting research strategies accordingly will improve user perceptions of researchers as being valued partmers and not just as (one of many) suppliers.

A focus on the other pole of the intelligence service encounter, i.e. the decisionmaker, completes our vision on international service intelligence effectiveness. Our results suggest that subjective decision-maker attitudes towards an international service-related topic will affect cognitions and behavior concerning service intelligence regarding this topic. Intentions of selectively using intelligence were found to be related to the strength with which these attitudes are being held. Especially attitude commitment, representing user certainty that one's opinion is correct and the 
willingness to take a strong position in defending that belief, is a significant determinant of international service intelligence effectiveness. More specifically, intelligence is likely to be more effective when it is in line with user attitudes towards the intelligence issue. Researchers, therefore, need to develop an understanding of how users think or feel about a specific topic under investigation. Costly intelligence can be made more effective, either by adapting it to user attitudes or, from a more ethical perspective, help to develop user attitudes that are in line with the intelligence. Furthermore, by knowing how committed users are towards an international service issue, the extend to which they think about this issue can be predicted, as well as deeper insights can be developed into the expectations users have with respect to intelligence dealing with the issue. This enables intelligence providers to further enhance international service intelligence effectiveness.

We are currently witnessing a gargantuan rise in connectivity and subsequent information availability as a result of open and virtually cost-free exchange of increasingly rich information. Intelligence, as a result, may increasingly be the end product for a majority of businesses as it glues together value as well as supply chains and networks of organizations. In the age of the economics of information the challenge becomes to make intelligent use of intelligence.

\subsection{A Perspective on Future Research}

We conclude this thesis by looking to the road ahead. The infusion of technology is rapidly resulting in interconnected market places that are characterized by a tremendous free-flow of information moving back and forth between individual actors. As a consequence, computer-mediated communication is becoming a strategic tool for business organizations. As a result, extensive amounts of information are exchanged leading to a redefinition of strategies for generating effective international service intelligence. It is within this context that future research priorities on intelligence effectiveness are taking shape.

As we have already seen, advances in technology have substantial effects on international services and their service delivery modes. Likewise, they can be expected to change the ways in which international service intelligence can be generated in the future. For instance, the internet offers an increasing range of opportunities for collecting data and conducting marketing research in international services settings. Online survey methods are being developed for generating intelligence on and from service customers. New interactive technological developments create an onine 
environment in which customers are able to express perceptions, opinions, and desires both verbally as well as nonverbally. Visualized aids can supplement traditional, verbal-oriented tools for collecting intelligence and assist researchers in overcoming difficulties often related to cross-cultural differences in language and communication between respondents. Difficulties, however, remain with respect to the interpretation of such non-verbal cues and undoubtedly merit further investigation.

Increased interconnectivity through online social as well as business networks enables service firms to actively communicate with customers and, consequently, obtain information directly from them. Intelligence in the information age will become more personal, containing information pertaining to personal and professional wellbeing. As a consequence, international personalized market segments can now be identified by service firms. In addition, customers are also increasingly able to communicate with each other. Entire communities of (service) customers seem to emerge in cyberspace, regardless of geographical and physical boundaries. In these virtual communities, customers can 'meet' and exchange information involving ideas, opinions, perceptions, etc. The richness of the intelligence that is surfacing through such media is of utter strategic importance to international service firms, especially since it pre-eminently originates from customers themselves. Furthermore, service organizations will also be able to 'link up' with other service firms through the infusion of technology. Channel integration is likely to lead to the establishment of partnerships and inter-organizational exchange of intelligence. Future research should take these various types of interconnective intelligence into consideration and clarify ways to maximize its effectiveness for decision-making in international service settings.

Future research is likely to benefit from observing actual interactions between providers and users of marketing intelligence in order to develop a more adequate operationalization of the concept of intelligence effectiveness. Particularly, as a result of more advanced research techniques and the wider availability of research data, it will become gradually feasible to examine actual use of international service intelligence and compare it to intended use. Additional research should be directed at developing and listing valid and reliable measures of a more advanced metric of intelligence effectiveness.

Another direction for further research on intelligence effectiveness in international services is to establish connectivity with other, related research streams. For instance, in distribution research there has been an accumulating body of evidence on the nature and impact of various influence strategies and tactics. As we demonstrated, political acceptability is a key informational attribute in determining inteligence effectiveness. On the basis of this we feel it could be argued that a case could be made 
for the inclusion of social and political influence strategies for the actual use of intelligence (e.g., the coercive and non-coercive mechanisms a company headquarters have for influencing the attitudes and behavior of its international operating companies). We feel, therefore, that a fruitfull path for further research would be to expand on the impact of various influence strategles in conjunction with intelligence effectiveness in an international service context. Furthermore, additional variables originating from the relationship marketing as well as the service marketing literature may be welcomed as alternative explanations for explaining effectiveness of intelligence in the international services context.

We argued as well as empirically demonstrated that cross-cultural variation has an impact on the content of international service intelligence. Similarly, it could be reasoned that the symbolic significance of intended and actual information use depends on the cultural context in which it is taken. In other words, it may very well be that cross-culturally dependent interaction effects impact the use of intelligence by decision-makers. Future research should be undertaken to provide more clarity on this topic.

A final word of caution is warranted. The focus throughout the various projects presented in this dissertation was on international service intelligence and intelligence use in one specific industry, for one specific company. For the purpose of generalization of results, the conceptual frameworks and hypotheses presented here need to be validated in other settings and across other industries as well.

Uitimately, a more in-depth insight into the concept of intelligence effectiveness may provide a starting point for developing measures of organizational learming competency or knowledge management efficacy. In this way, verifiable links between intelligence effectiveness and organizational effectiveness could empirically be established. 



\section{References}

A

Abelson, R.P. (1988), "Conviction", American Psychologists, 43, 267-275.

Anderson, E.W. (1996), "Customer Satisfaction and Price Tolerance", Marketing Letters, $7(3), 19-30$.

Anderson, E.W. \& C. Fornell (1994), "A Customer Satisfaction Research Prospectus," in:

R.T. Rust \& R.L. Oliver (eds.), Service Quality: New Directions in Theory and Practice, Sage Publications, Thousand Oaks, 1-19.

Anderson, J.C. \& D.W. Gerbing (1988), "Structural Equation Modeling in Practice: A Review and Recommended Two-step Approach", Psychological Bulletin, 103, 411423.

B

Bagozzi, R.P. (1980), "Performance and Satisfaction in an Industrial Sales Force: An Examination of their Antecedents and Simultaneity", Journal of Marketing, 44 (Spring), 65-77.

Bagozzi, R.P. \& Y. Yi (1988), "On the Evaluation of Structural Equation Models", Journal of the Academy of Marketing Science, 16 (Spring), 74-94.

Barabba, V. (1978), Knowledge Utilization: The Difference Between the Public and Private

Sector, Paper Presented at the Conference on Federal Information Sources, Ottawa,

Canada, December 4-5.

Barabba, V.P. \& G. Zaltman (1991), Hearing the Voice of the Market: Competitive Advantage Through Creative Use of Market Information. Harvard Business School Press, Boston, MA.

Barnes, J.G., P.A. Dunne \& W.J. Glynn (2000), "Self-Service and Technology: Unanticipated and Unintended Effects on Customer Relationships," in: T.A. Swartz \& D. lacobucci (eds.), Handbook of Services Marketing \& Management, Sage Publications, inc., Thousand Oaks, etc., 89-102.

Bateson, J.E.G. (1995), Managing Services Marketing: Text and Readings, The Dryden Press, Fort Worth, etc.

Beatty, 5.E. \& S.M. Smith (1987). "External Search Effort: An Investigation Across Several Product Categories", Journal of Consumer Research, 24 (1), 83-95.

Bentler, P.M. (1990), "Comparative Fit Indexes in Structural Models", Psychological Bulletin, 61, 238-246.

Bentler, P.M. \& D.G. Bonett (1980), "Significance Tests and Goodness-of-Fit in the Analysis of Covariance Structures", Psychological Bulletin, 88, 588-606. 
Bentller, P.M. \& C. Chou (1987), "Practical Issues in Structural Modeling", Sociological Methods \& Research, 16 (August), 78-117.

Birgellen, M. van, K. de Ruyter \& M. Wetzels (2000), "An Empirical Investigation into Total Quality Management Information Use", Total Quality Management, in press. Bitner, M.J. (1990), "Evaluating Service Encounters: The Effects of Physical Surroundings and Employee Responses", Journal of Marketing, 54 (April), 69-82.

Bitner, M.J. (1995), "Building Service Relationships; It's All About Promises", Journal of the Academy of Marketing Science, $23(4), 246-251$.

Bitner, M.J., S.W. Brown \& M.L. Meuter (2000), "Technology Infusion in Service Encounters", Journal of the Academy of Marketing Science, 28 (Winter), 138-149.

Bollen, K.A. (1989), Structural Eqwations with Latent Variables, John Wiley \& Sons, New York.

Brace, C. (2000), "Tomorrow Calling", Holland Herald, September, 65-94.

Bradley, F. (1995), "The Service Firm in International Marketing", in: W.J. Glynn \& J.G.

Barnes (eds.), Understanding Services Management, John Willey \& Sons; Chicester, $420-4.48$.

Bradley, F. (1999), International Marketing Strategy, Prentice Hall, London, etc.

Brislin, R.W. (1980). "Translation and Content Analysis of Oral and Written Materials",

in: H.C. Triandis \& J.W. Berry (eds.), Handbook of Cross-Cultural Psychology, Vol. 2: Methodology, Allyn and Bacon, Boston, 389-444.

Bryk, A.S. \& S.W. Raudenbush (1992), Hierarchical Linear Madels: Applications and Data Analysis Methods, Sage Publications, Newbury Park, CA.

Buzzell, R.D. (1968), "Can you Standardize Multinational Marketing?", Harvard Business Review, 46 (November-December), 102-113.

Buzzell, R.D. \& B.T. Gale (1987). The PIMS Principles: Linking Strategy to Performance, The Free Press, New York/London.

Buzzell, R.D., B.T. Galle \& R.G.M. Sultan (1975). "Market Share: A Key to Profitability", Harvard Business Review, 53 (January-February), 97-106.

Byrne, B.M., R.J. Shavelson \& B. Muthén (1989), "Testing for the Equivalence of Factor Covariance and Mean Structures: The Issue of Partial Invariance", Psychological Bulletin, 105, 456-466.

C

Carlson, J.R. \& R.W. Zmud (1999). "Channel Expansion Theory and the Experiential Nature of Media Richness Perceptions". Academy of Management Journal, 42 (2). $153-170$. 
Chaiken, 5. (1980), "Heuristic Versus Systematic Information Processing and the Use of Source Versus Message Cues in Persuasion", Joumal of Personality and Social Psychology, 39, 752-766.

Chaiken, S. \& S. Yates (1985), "Affective-Cognitive Consistency and Thought-1nduced Attitude Polarization", Journal of Personality and Social Psychology. 49, 1470-1481.

Chaiken, S., E.M. Pomerantz \& R. Giner-Sorolla (1995), "Structural Consistency and Attitude Strength", in: R.E. Petty \& J.A. Krosnick (eds.) Attitude Strength: Antecedents and Consequences, Lawrence Erlbaum Associates, Mahwah, NJ, 387412 .

Cohen, 1. (1992), "A Power Primer", Psychological Bulletin, 112 (1), 155-159.

Cohen, M.S. (1989), "A Database Tool to Support Probabilistic Assumption-based Reasoning in Intelligence Analysis", Proceedings of the 1989 Joint Director of the C2 Symposium, Ft. McNair, VA, (June), 27-29.

Cowell, D. (1993), The Marketing of Services, Heinemann, London.

Craig, C.S. \& S.P. Douglas (2000), International Marketing Research, John Wiley \& Sons, Ltd., Chichester, etc.

Cronin Jr., J.J. \& S.A. Taylor (1992), "Measuring Service Quality: A Reexamination and Extension", Journal of Marketing, 56, 55-68.

Czinkota, M.R. \& I.A. Ronkainen (1995), Intermational Marketing, The Dryden Pres5, Fort Worth, etc.

Dabholkar, P.A. (1992), "Role of Affect and Need for Interaction in On-site Service Encounters", Advances in Consumer Research, 19, 563-569.

Dabholkar, P.A. (1994), "Technology-based Service Delivery: A Classification Scheme for Developing Marketing Strategies". Advances in Services Marketing and Management, 3, 241-271.

Dabholkar, P.A. (1996), "Consumer Evaluations of New Technology-Based Self-Service Options: An Investigation of Alternative Models of Service Quality", International Journal of Research in Marketing, 13 (1), 29-51.

Dabholkar, P.A. (2000), "Technology in Service Delivery: Implications for Self-Service and Service Support", in: T.A. Swartz \& D. lacobucci (eds.), Handbook of Services Marketing \& Management, Sage Publications, Inc., Thousand Oaks, etc., 103-110.

Dabholkar, P.A., C.D. Shepherd \& D.1. Thorpe (2000), "A Comprehensive Framework for Service Quality: An Investigation of Critical Conceptual and Measurement issues Through a Longitudinal Study" , Journal of Retailing, 76 (2), 139-173.

Dahringer, L.D. (1991), "Marketing Services Internationally: Barriers and Management" Strategies", Journal of Services Marketing, 5 (3), 5-17. 
Davidson, A.R., S. Yantis, M. Norwood \& D.E. Montano (1985). "Amount of Information about the Attitude Object and Attitude-Behaviar Consistency", Joumal of Personality and Social Psychology, 49, 1184-1198.

Dawar, N. \& P. Parker (1994), "Marketing Universals: Consumers' Use of Brand Name, Price, Physical Appearance and Retailer Reputation as Signals of Product Quality", Journal of Marketing, 58 (April), 81-95.

Dempster, A.P., N.M. Laird \& D.B. Rubin (1977), "Maximum Likelihood from Incomplete Data via the EM Algorithm", Journal of Royal Statistical Association, 76, 341-353.

Deshpandé, R. (1989), "How Decision Makers Use Marketing Information", in: 5. Krishnan (ed.), Making More Effective Use of Market information: A Conference Summary, Report No. 89-113, Marketing Science Institute, Cambridge, MA.

Deshpandé, R. \& G. Zaltman (1982), "Factors Affecting the Use of Market Research Information: A Path Analysis", Journal of Marketing Research, 19 (February), 14-31.

Deshpandé, R. \& G. Zaltman (1984), "A Comparison of Factors Affecting Researcher and Manager Perceptions of Market Research Use", Journal of Marketing Research, 21 (February), 32-38.

Deshpandé, R. \& G. Zaltman (1985). "The Use of Market Research in Industrial Organizations" "in: R. Spekman \& D. Wilson (eds.). A Strategic Approach to Business Marketing, American Marketing Association, Chicago, 58-66.

Deshpandé, R. \& G. Zaltman (1987), "A Comparison of Factors Affecting Use of Marketing Information in Consumer and Industrial Firms", Journal of Marketing Research, 24 (February), 114-118.

Dickerson, M.D. \& J.W. Gentry (1983), "Characteristics of Adopters and Non-Adopters of Home Computers", Journal of Consumer Research, 10 (September), 225-235.

Donthu, N. \& B. Yoo (1998), "Cultural Influences on Service Quality Expectations", Journal of Service Research, 1 (2), 178-186.

Douglas, S.P. \& C.S. Craig (1983), International Marketing Research, Prentice Hall, Englewood Cliffs, N.J.

E

Eagly, A.H. \& S. Chaiken (1993), The Psychology of Attitudes, Harcourt Brace Jovanovich College Publishers, Fort Worth, TX.

Eagly, A.H. \& S. Chaiken (1995), "Attitude Strength, Attitude Structure, and Resistance to Change", in: R.E. Petty \& J.A. krosnick (eds.), Attitude Strength: Antecedents and Consequences, Lawrence Erlbaum Associates, Mahwah, NJ, 413-432.

Elder, J.W. (1976), "Comparative Cross-national Methodology", Annual Review of Sociology, 2 , Annual Reviews, Inc., Palo Alto, CA. 
Ernst, LA. (1976), "703 Reasons Why Creative People Don't Trust Research", Advertising Age, 10 (February), 35-36.

F

Feldman, M.S. \& J.G. March (1981), "Information in Organizations as Signal and Symbol" "Administrative Science Quarterly, 26 (2), 171-186.

Fishbein, M. \& I. Ajzen (1975), Belief, Attitude, Intention, and Behavior: An Introduction to Theory and Research, Addison-Wesley, Reading MA.

Fisher, L.M. (1998), "Here Comes Front-Office Automation", Strategy and Business, 13 (4), 53-65.

Fiske, S.T. \& S.E. Taylor (1991), Social Cognition, McCraw-Hill, New York.

Folkes, V.A. (1994), "How Consumers Predict Service Quality: What do they Expect", in: R.T. Rust \& R.L. Oliver (eds.), Service Quality, New Directions in Theory and Practice, Sage Publications Inc., California, 108-122.

Ford, G.T. \& R.A. Smith (1987), "Inferential Beliefs in Consumer Evaluations: An Assessment of Alternative Processing Strategies", Journal of Consumer Research, 14 (December), 363-371.

Fornell, C. \& D.F. Larcker (1981), "Evaluating Structural Equation Models with Unobservable Variables and Measurement Error" Journal of Marketing Research, 18 (February), 19-50.

Forrest, E. (1999), Internet Marketing Research: Resources and Techniques, The McGrawHill Companies, Inc., Sydney, etc.

Fulk, 1. (1993), "Social Construction of Communication Technology", Academic Management Journal, 36, 921-950.

Fulk, J. \& B. Boyd (1991), "Emerging Theories of Communication in Organizations", Journal of Management, 17, 407-446.

Furrer, O., B.S-C. Liu \& D. Sudharshan (2000), "The Relationships between Culture and Service Quality Perceptions: Basis for Cross-cultural Market Segmentation and Resource Allocation", Journal of Service Research, 2 (4), 355-371.

G

Gallois, C. \& V.J. Callan (1997), Communication and Culture: A Guide for Practice, John Wiley \& Sons, Chichester, etc.

Gardial, S. \& G. Biehal (1987), "Measuring Consumer's Inferential Processing in Choice", in: E.C. Hirschmann \& M.B. Holbrook (eds.). Advances in Consumer Research, Association for Consumer Research, Ann Arbor, MI, 14, 101-105. 
Gerbing, D.W. \& J.C. Anderson (1988), "An Updated Paradigm for Scale Development Incorporating Unidimensionality and its Assessment", Journal of Marketing Research, 25 (May), 186-192.

Gilbert, D.T. B.W. Pelhan \& D.S. Krull (1988), "On Cognitive Business: When Person Perceivers meet Persons Perceived", Journal of Personality and Social Psychology, 54 . $733-740$.

Goldstein, H. (1995), Multilevel Statistical Madels, Griffin, London.

Greenwald, A.G. \& C. Leavitt (1984), "Audience Involvement in Advertising Four Levels". Journal of Consumer Research, 11, 581-592.

Grudin, J. (1994), "Groupware and Social Dynamics: Eight Challenges for Developers", Communications of the ACM 37, 1, 92-105.

Guseman, D.S. (1981), "Risk Perception and Risk Reduction in Consumer Services", in: J.H. Donnelly \& W.R. George (eds.), Marketing of Serwices, American Marketing Association, Chicago, 200-204.

H

Hayduk, L.A. (1987), Structural Equation Modeling with LISREL: Essentials \& Advances, The $\downarrow$ ohn Hopkins University Press, Baltimore \& London.

Heim, G.R. \& K.K. Sinha (2000), "Design and Delivery of Electronic Services: Implications for Customer Value in Electronic Food Retailing", in: J.A. Fitzsimmons \& M.J. Fitzsimmons (eds.), New Service Development: Creating Memorable Experiences, Sage Publications, Inc., Thousand Oaks, etc, 152-182.

Hiltz, S.R. \& K. Johnson (1989), "Measuring Acceptance of Computer-Mediated Communication Systems" , Journal of the American Society for Information Science, $40(6), 386-397$.

Hoelter, J.W. (1983), "The Analysis of Covariance Structures: Goodness-of-Fit Indices", Sociological Methods and Research, 11 (February), 325-344.

Hofmann, D.A. (1997), "An Overview of the Logic and Rationale of Hierarchical Linear Models", Journal of Management, 23, 723-744.

Hofstede, G. (1980), Culture's Consequences: International Differences in Work-related Values, Sage Publications, Beverly Hills, CA.

Hofstede, G. (1991), Cultures and Organizations: Software of the Mind, McGraw-Hill, London.

Hogarth, R.M. \& S. Makridakis (1988), "Forecasting and Planning: An Evolution", in: P.E.

Earl (ed.), Behovioural Economics, Schools of Thought in Economics Series, 1 (6), Elgar, Aldershot, UK/Gower, Brookfield, VT.

Hoppe, M.H. (1990), A Comparative Study of Country Elites, Doctorall Dissertation, University of North Carolina, Chapel Hill. 
Houston. D.A. \& R.H. Fazio (1989), "Biased Processing as a Function of Attitude Accessibility: Making Objective Judgments Subjectively", Social Cognition, 7, 51-66. Hox, J.J. (1995), Applied Multilevel Analysis, T.T.-Publikaties, Amsterdam.

Hox, J.J. \& I.G.G. Kreft (1994), "Multilevel Analysis Methods", Sociological Methods \& Research, 22, 283-299.

Hu, L. \& P.M. Bentler (1995). "Evaluating Model Fit", in: R.H. Hoyle (ed.), Structural Equation Modeling: Concepts, Issues, and Applications, Sage Publications, Thousand Oaks, 76-99.

Huber, J. \& J. McCann (1982), "The Impact of inferential Beliefs on Product Evaluations", Journal of Marketing Research, 19 (August), 324-333.

Huber, F., A. Herrmann \& Ch. Braunstein (1997). "Standardization and Differentiation of Services: A Crosscultural Study Based on Semiotics, Means End Chains and Conjoint Analysis", in: Ashford et al. (eds.), Marketing Across Borders, Conference Proceeding, The Manchester Metropolitan University, Manchester, 219-234.

Jaccard, 1. (1981), "Attitudes and Behavior: Implications of Attitudes toward Behavioral Alternatives", Journal of Experimental Social Psychology, 17, 286-307.

Jaccard, J. \& M.A. Becker (1985). "Attitudes and Behavior: An Information Integration

Perspective", Journal of Experimental Social Psychology, 21, 440-465.

Jaccard, I., C. Radecki, T. Wilson \& P. Dittus (1995), "Methods for Identifying Consequential Beliefs: Implications for Understanding Attitude Strength", in: R.E. Petty \& J.A. Krosnick (eds.), Attitude Strength: Antecedents and Consequences, Lawrence Erlbaum Associates, Mahwah, NI, 337-359.

Jagacinski, C.M. (1991), "Personnel Decision Making: The Impact of Missing Information", Journal of Applied Psychology, $76(1), 19-30$.

Johansson, J.K. (1997), Global Marketing: Foreign Entry, Local Marketing, and Global Management, R.D. Irwin, Chicago.

Johnson, R.D. (1989), "Making Decisions with incomplete Information: The First Complete Test of the Inference Model", in: T.K. Srull (ed.), Advances in Consumer Research, 16, Association for Consumer Research, Provo, UT, 522-528.

Johnson, R.D. \& I.P. Levin (1985), "More than Meets the Eye: The Effect of Missing Information on Purchase Evaluations", Journal of Consumer Research, 12 (September), 169-177. Jones, 5. \& 5. Marsh (1997), "Human-Computer-Human Interaction: Trust in CSCW", SICCHIB Bulletin, $29(3), 36-40$.

Jöreskog, K.G. (1971a), "Simultameous Factor Analysis in Several Populations", Psychometrika, 36 (December), 409-426. 
Jöreskog, K.G. (1971b), "Statistical Analysis of Sets of Congeneric Tests", Psychometrika, 36 (June), 109-133.

Jöreskog, K.G. \& D. Sörbom (1993), LSREL 8: Structural Equation Modeling with the SIMPLS Command Language, Scientific Software International, Hillsdale/Lawrence Erlbaum Associates, Chicago.

K

Kahneman, D., P. Slovic \& A. Tversky (Eds.) (1982), Judgment under Uncertainty: Heuristics and Biases, Cambridge University Press, Cambridge, MA.

Keppel, G. (1991), Design and Analysis: A Researcher's Handbook, Prentice-Hall, Englewood Cliffs, NJ.

Kirk, R.E. (1982), Experimental Design: Procedures for the Behavioral Sciences, Brooks/Cole, Monterey, CA.

Kleinbaum, D.G., L.L. Kupper \&. K.E. Muller (1988), Applied Regression Analysis and Other Multivariable Methods, PWS-KENT Publishing Company, Boston.

Kluckhohn, C. (1951), "The Study of Culture", in: D. Lerner \& H.D. Lasswell (eds.), The Policy Sciences, Stanford University Press, Stanford, CA.

Kohli, A. \& B.J. Jaworski (1990), Market Orientation: The Construct, Research Propositions, and Managerial Implications, Report No. 90-113, Marketing Science Institute, Cambridge, $M A$.

Kotler, Ph. \& G. Armstrong (1997), Marketing: An Introduction, Prentice Hall, Upper Saddle River, NJ.

Kreft, I.G.G. \& I. de Leeuw (1998), Introducing Multilevel Modeling, Sage Publications, London/Thousand Oaks, New Delhi.

Krishnan, S. (1989), Making More Effective Use of Market Information: A Conference Summary, Report No. 89-113, Marketing Science Institute, Cambridge, MA.

Krosmick. J.A. (1988), "Attitude Importance and Attitude Change", Journal of Experimental Social Psychology, 24, 240-255.

Krosnick, J.A. (1990), "American's Perceptions of Presidential Candidates: A Test of the Projection Hypothesis", Journal of Social /ssues, 46, 159-182.

Krosnick, J.A. \& R.E. Petty (1995). "Attitude Strength: An Overview" in: R.E. Petty \& J.A. Krosnick (eds.), Attitude Strength: Antecedents and Consequences, Lawrence Erlbaum Associates, Mahwah, NJ.

Krosnick, J.A., D.S. Boninger, Y.C. Chuang, M.K. Berent \& C.G. Carnot (1993), "Attitude Strength: One Construct or Many Related Constructs?", Journal of Personality and Social Psychology, 65 (6), 1132-1151. 
Kumar, A. \& W.R. Dillion (1987), "The Interaction of Measurement and Structure in Simultaneous Equation Models with Unobservable Variables", Journal of Marketing Research, 24 (February), 98-105.

$\underline{L}$

Lastovika, J.L. \& D.M. Gardner (1979), "Components of Involvement", in: I.C. Maloney \& B. Silverman (eds.), Attitude Research Plays for High Stakes, American Marketing Association, Chicago.

Ledingham, J.A. (1984), "Are Consumers Ready for the Information Age?", Journal of Advertising Research, $24(4), 31-37$.

Lee, H., F. Acito \& R.L. Day (1987), "Evaluation and Use of Marketing Research by Decision Makers: A Behavioral Simulation" Journal of Marketing Research, 24 (May), $187-196$.

Lee, H., I.D. Lindquist \& F. Acito (1997), "Managers" Evaluation of Research Design and its Impact on the Use of Research: An Experimental Approach", Joumal of Business Research, 39, 231-240.

Lee, H., P.M. Herr, F.R. Kardes \& C. Kim (1999), "Motivated Search: Effects of Choice Accountability, Issue Involvement, and Prior Knowledge on Information Acquisition and Use", Journal of Business Research, 45, 75-88.

Lim, J.-S. \& J. Kim (1992), "Impact of Consumers" Confidence in Judgements about Missing Information on Product Evaluations", Journal of Business Research, 25, 215229.

Lipe, M.G. (1998), "Individual Investor's Risk Judgments and Investment Decisions: The Impact of Accounting and Market Data", Accounting, Organizations and Society, 23 $(7), 625-640$.

Lipshitz, R. \& O. Ben Shaul (1997), "Schemata and Mental Models in Recognitionprimed Decision-making", in: C. Zsambok \& G.A. Klein Keds.), Naturalistic Decisionmaking. Erlbaum, Hillsdale, NJ, 292-303.

Lipshitz, R. \& O. Strauss (1997), "Coping with Uncertainty: A Naturalistic Decisionmaking Analysis", Organizational Behavior and Human Decision Processes, 69 (2) (February), 149-163.

Loehlin, J.C. (1998), Latent Variable Models: An Introduction to Factor, Path, and Structural Analysis, Lawrence Erlbaum Associates, Mahwah, NJ.

Lord, C.G." L. Ross \& M.R. Lepper (1979), "Biased Assimilation and Attitude Polarization: The Effects of Prior Theories on Subsequently Considered Evidence", Journal of Personality and Social Psychology, 37, 2098-2109.

Lovelock, C. \& G. Yip (1996), "Developing Global Strategies for Service Businesses", California Management Review, 38, 64-86. 
Lynn, M. \& B.D. Gelb (1996), "Identifying Innovative National Markets for Technical Consumer Goods" "International Marketing Review, 13 (December), 43-57.

Lynn, M. G.M. Zinkhan \& J. Harris (1993), "Consumer Tipping: A Cross-Country Study", Journal of Consumer Research, 20 (December), $478 \times 488$.

\section{M}

Maheswaran, D. \& J. Meyers-Lewy (1990), "The Influence of Message Framing and Issue Involverment", Journal of Marketing Research, 27,361-367.

Maheswaran, D. \& B. Sternthal (1990), "The Effects of Knowledge, Motivation, and Type of Message on Ad Processing and Product Judgements", Journal of Consumer Research, 17, 66-73.

Maltz, E. \& A.K. Kohli (1996), "Market Intelligence Dissemination across Functional Boundaries", Journal of Marketing Research, 33 (February), 47-61.

Maney. K. (1997), "Technology is 'Demolishing" Time, Distance", USA Today, 24 (April), 1B-2B.

Marks, L.J. \& M.A. Kamins (1988), "The Use of Product Samples and Advertising: Effects of sequences of exposure and Degree of Advertising Claims Exaggeration on Consumers" Beliefs Strength, Belief Confidence, and Attitudes" , Journal of Marketing Research, 25, 266-281.

Marsh, H.W. (1994), "Confirmatory Factor Analysis Models of Factorial Invariance: A Multifaceted Approach", Structural Equation Modeling, 1 (1), 5-34.

Marsh, H.W. \& D. Hocevar (1985), "Application of Confirmatory Factor Analysis to the Study of Self-Concept: First- and Higher Order Factor Models and Their Invariance Across Groups", Psychological Bulletin, 97 (3), 562-582.

Marsh, H.W. J.R. Balla \& R.P. McDonald (1988), "Goodness-of-fit Indexes in Confirmatory Factor Analysis: The Effect of Sample Size" ${ }^{*}$ Psychological Bulletin. 103, 391-410.

Marsh, H.W., J.R. Balla \& H.K. Tai (1996), "An Evaluation of Incremental Fit Indices: A Clarification of Mathematical and Empirical Properties", in: G.A. Marcoulides \& R.E. Schumacker (eds.), Advanced Structural Equation Modeling: Issues and Techniques. Lawrence Erlbaum Associates, Mahwah, NJ.

Mattila, A.S. (1999), "The Role of Culture in the Service Evaluation Process", Journal of Service Research, $1(3), 250-261$.

McCaskey, M.B. (1986), The Executive Challenge: Manage Change and Ambiguity. Harper Business.

McDaniel, M.A., E.L. DeLosh, G.D. Einstein, C.P. May \& P. Bracy (1995), "The Bizarreness Effect: It's not Surprising, it's Complex", Journal of Experimental Psychology: Learning. Memory and Cognition, 21, 422-435. 
Menon, A. \& R. Varadarajan (1992), "A Model of Marketing Knowledge Use within Firms", Journal of Marketing, 56 (October), 53-71.

Menon, A. \& J. Wilcox (1994), USER: A Scale to Measure Use of Market Research, Report No. 94-108, Marketing Science institute, Cambridge, MA.

Meuter, M.L., A.L. Ostrom, R.I. Roundtree \& M.J. Bitner (2000), "Self-Service Technologies: Understanding Customer Satisfaction with Technology-Based Service Encounters", Journal of Marketing, 64 (3), 50-64.

Mick, D.G. \& S. Foumier (1998), Paradoxes of Technology: Consumer Cognizance, Emotions, and Coping Strategies, Working paper, Report No. 98-112, July, Marketing Science Institute, Cambridge, MA.

Miller, A.G., J.W. McHoskey, C.M. Bane \& T.G. Dowd (1993), "The attitude Polarization Phenomenon: Role of Response Measured Attitude Extremity, and Behavioral Consequences of Reported Attitude Change", Journal of Personality and Social Psychology, 64, 561-574.

Moorman, Ch. \& J. Austin (1995), The Paradox of Low Quality and High Use: How Research Trust impacts Market Research Outcomes, Report No. 95-116, Marketing Science Institute, Cambridge, MA.

Moorman, Ch., R. Deshpandé \& G. Zaltman (1993). "Factors Affecting Trust in Market Research Relationships", Journal of Marketing, 57 (January), 81-101.

Moorman, Ch., G. Zaltman \& R. Deshpandé (1992), "Relationships between Providers and Users of Market Research: The Dynamics of Trust within and between Organizations", Journal of Marketing Research, 29 (August), 314-328.

Mowen, J.C. (1995), Consumer Behavior, $4^{\text {th }}$ ed., Prentice Hall, Englewood Cliffs, NJ. Mulder, P. (1999), "Er Verandert Geen Moer!": Frustraties met Continuklanttevredenheidsonderzoek", Tjjdschrift woor Marketing, (April), 20-24.

Mullen, M. (1995), "Diagnosing Measurement Equivalence in Cross-national Research". Journal of International Business Studies, 26 (3), 573-596.

N

Netemeyer, R.G., M.W. Johnston \& S. Burton (1990), "Analysis of Role Conflict and Role Ambiguity in a Structural Equations Framework", Joumal of Applied Psychology, 75. 148-157.

Neter, J., W. Wasserman \& M.H. Kutner (1990), Applied Linear Statistical Models: Regression, Analysis of Variance and Experimental Designs, Irwin, Burr Ridge, IL.

Newman, J.W. (1962), "Putting Research into Marketing Decisions", Harvard Business Review, March-April, 105-112.

Newman, L.S. \& J.S. Uleman (1989), "Spontaneous Trait Inference", in: J.5. Uleman \& I.A. Barge (eds.), Unintended Thought, Guilford, New York, 155-188. 
Nunnally, J.C. \& I.H. Bernstein (1994), Psychometric Theory, McGraw-Hill, Inc., New Vork, etc.

Nylen, D.W. (1990), Marketing Decision-Making Handbook, Prentice Hall, Englewood Cliffs, N.J.

O

Oliver, R.L. (1980), "A Cognitive Model of the Antecedents and Consequences of Satisfaction Decisions", Journal of Marketing Research, 17 (November), 460-469.

Oliver, R.L. (1993), "A Conceptual Model of Service Quality and Service Satisfaction: Compatible Goals, Different Concepts", in: A.T. Swartz, D.E. Bowen \& S.W. Brown (eds.), Advances in Services Marketing Management. JAl Press, Greenwich, CT, 2, 6585.

Oliver, R.L. (1997), Satisfaction: A Behavioral Perspective on the Consumer, McGraw-Hill, Inc., New York.

Olson, J.M. \& M.P. Zanna (1993), "Attitudes and Attitude Change", Annual Review of Psychology, 44, 117-154.

Orasanu, J. \& T. Connolly (1993), "The Reinvention of Decision-making", in:, G.A. Klein, J. Orasanu, R. Calderwood \& C. Zsambok (eds.), Decision-making in Action: Models and Methods, Ablex Publishing, Norwoad, NJ, 3-20.

\section{P}

Parasuraman, A. (1996), "Understanding and Leveraging the Role of Customer Service in External, Interactive, and Internal Marketing", Frontiers in Services Conference, Nashville, TN, October 5 .

Parasuraman, A (2000), "Technology Readiness Index (TRI): A Multiple-Item Scalle to Measure Readiness to Embrace New Technologies", Journal of Service Research, 2 (4), $307-320$.

Parasuraman, A., V.A. Zeithaml \& L.L. Berry (1985), "A Conceptuall Model of Service Quality and its Implications for Future Research", Journal of Marketing, 49 (Fall), 4150.

Parasuraman, A., V.A. Zeithaml \& L.L. Berry (1988), "SERVQUAL: A Multiple-item Scale for Measuring Consumer Perceptions of Service Quality", Journal of Retailing, 64 (Spring), $12-40$.

Parasuraman, A., V.A. Zeithaml \& L.L. Berry (1994), "Reassessment of Expectations as a Comparison Standard in Measuring Service Quality: Implications for Further Research", Journal of Marketing, 58, 111-124.

Peppers, D. \& M. Rogers (1997), Enterprise One to One: Tools for Competing in the Interactive Age, Doubleday, New York. 
Perdue, B.C. \& J.O. Summers (1986), "Checking the Success of Manipulations in Marketing Experiments", Journal of Marketing Research, 23 (Nowember), 317 -326.

Perkins, W.S. \& R.C. Rao (1990), "The Role of Experience in Information Use and

Decision Making by Marketing Managers", Joumal of Marketing Research. 27 (February), 1-10.

Petty, R.E. \& J.T. Cacioppo (1979), "Issue Involvement can Increase or Decrease

Persuasion by Enhancing Message-Relevant Cognitive Response", Journal of Personality and Social Psychology, 37, 1915-1926.

Petty, R.E. \& J.T. Cacioppo (1986a), Communication and Persuasion Central and Peripheral Routes to Attitude Change, Springer-Verlag, New York.

Petty, R.E. \& J.T. Cacioppo (1986b), "The Elaboration Likelihood Model of Persuasion", in: L. Berkowitz (ed.), Advances in Experimental Social Psychology, Orlando, FL.

Petty, R.E., J.T. Cacioppo \& D. Schumann (1983), "Central and Peripheral Routes to Advertising Effectiveness: The Moderating Role of involvement" , Journal of Consumer Research, 10, 135-146.

Pligt, J. van der, P. Ester \& J. van der Linden (1983). "Attitude Extremity, Consensus, and Diagnosticity", European lournal of Social Psycholagy, 13. 437-439.

Pomerantz, E.M. S. Chaiken \& R.S. Tordesillas (1995), "Attitude Strength and Resistance Processes", Journal of Personality and Social Psychology, 69 (3), 408-419. Potter, R.E. \& L.R. Beach (1994) "Imperfect Information in Pre-choice Screening of Options", Organizational Behawior and Human Decision Processes, 59, 313-329.

Przeworski, A. \& H. Teune (1970), The Logic of Comparative Social Inquiry, Wiley Interscience, New York.

Quinn, J.B. (1996), "The Productivity Paradox is False: Information Technology lmproves Service Performance", in: T.A. Swartz, D.E. Bowen \&. S.W. Brown (eds.). Advances in Services Marketing and Management, JAl Press, Greenwich, CT, 5, 71-84.

$\underline{R}$

Raden, D. (1985), "Strength-Related Attitude Dimensions", Social Psychology Quarterly, $48(4), 312-330$.

Raphael, I. \& R. Parket (1991), "The Need for Market Research in Executive Decision Making", Journal of Business and Industrial Marketing, 6 (1/2) (Winter/Spring), 1521.

Rasbash, J. W. Browne, M. Healy, B. Cameron \& C. Charlton (1999), MLwIN BETA Version 1.10.0005. Multilevel Models Project, Institute of Education, London. 
Raudenbush, S.W. (1993). "Hierarchical Linear Models and Experimental Design", in:

L.K. Edwards (ed.), Applied Analysis of Variance in Behavioral Science, Marcel Dekker, Niew York, $459-496$.

Richardson, R. \&. J. Marshall (1999), "Teleservices, Call Centres, and Urban and Regional Development". The Service Industries Journal, 19 (1), 96-116.

Risch Rodie, A. \& 5. Schultz Kleine (2000), "Customer Participation in Services

Production and Delivery", in: T.A. Swartz \& D. lacobucci (eds.), Handbook of Services

Marketing \& Management, Sage Publications, Inc., Thousand Oaks, etc., 111-125.

Robinson, G.L. (1988), Cross-cultural Understanding, Prentice Hall, Sydney.

Ross Jr. W.T. \& E.H. Creyer (1992), "Making Inferences about Missing Information: The

Effects of Existing Information", Journal of Consumer Research, 19 (June), 14-25.

Roth, M.S. (1995), "The Effects of Culture and Socioeconomics on the Performance of

Global Brand Image Strategies", Journal of Marketing Research, 32 (May), 163-175.

Rust, R.T. (1998), "What is the Domain of Service Research?", Journal of Service Research, $1(2), 107$.

Rust, R. (1999), "Technology and Service", Editorial Journal of Service Research, 1 (3), 195.

Rust, R.T \& R.L. Oliver (1994), "Service Quality: Insights and Managerial Implications from the Frontier", in: R.T. Rust and R.L. Oliver (eds.), Service Quality: New Directions in Theory and Practice, Sage, London, 1-19.

Rust, R.T., A.J. Zahorik \& T.L. Keiningham (1995), "Return on Quality (ROQ): Making Service Quality Financially Accountable", Journal of Marketing, 59 (Aprill), 58-70.

Rust, R.T., A.J. Zahorik \& T.L. Keiningham (1996), Service Marketing. Harper Collins College Publishers, New York.

Rust, R.T., V.A. Zeithaml \& K.N. Lemon (2000), Driving Customer Equity: How Customer Lifetime Value is Reshaping Corporate Strategy. The Free Press, New York, etc.

Ruyter, K. de \& M. Wetzels (2000), "The Impact of Perceived Listening Behavior in Voiceto-Voice Service Encounters", Journal of Service Research, 2 (3), 276-284.

Ruyter, K. de, M. van Birgelen \& M. Wetzels (1998), "Consumer Ethnocentrism in International Services Marketing", International Business Review, 7, 185-202.

Ruyter, K. de, J. Bloemer \& P. Peeters (1997), "Merging Service Quality and Service Satisfaction: An Empirical Test of an Integrative Model", Journal of Economic Psychology, 18, 387-406.

Ruyter, K. de, M. Wetzels \& M. Kleijnen (2000), "Customer Adoption af E-Service: An Experimental Study" International Joumal of Service Industry Management, Forthcoming. 


\section{$\mathrm{S}$}

Sanbonmastu, D.M., S. Shavitt \& S.J. Sherman (1991)" The Role of Personal Relevance in the Formation of Distinctiveness-based Illusory Correlations", Personality and Social Psychology Bulletin, 17, 124-132.

Sawyer, A.G. \& A.D. Ball (1981), "Statistical Power and Effect Size in Marketing Research", Journal of Marketing, 17 (August), 275-290.

Schmitz, J. \& J. Fulk (1991), "Organizational Colleagues, Media Richness, and Electronic Mail: A Test of the Social Influence Model of Technology Use", Communication Research, $18(4), 563-598$.

Schoeffler, S., R.D. Buzzell \& D.F. Heany (1974), "Impact of Strategic Planning on Profit Performance" . Harvard Business Review, 56 (September-October), 104-114.

Schwartz, S.H. (1994), "Beyond Individualism/Collectivism: New Cultural Dimensions of Values", in: U. Kim et al. (eds.), Individualism and Collectivism: Theory, Method, and Applications, Sage Publications, Thousand Oaks, CA, 85-119.

Segal-Horn, S. (1994), "Are Service Industries Going Global?", in: C. Armistead (ed.), The Future of Services Management, Kogan Page, London, 41-63.

Shaked, 1. (1986), "Are Multinational Corporations Safer?", Journal of International Business Studies, 17 (Spring), 100.

Sherman, S.J., C.M. Judd \& B. Park (1989), "Social Cognition", Annual Review of Psychology, 40, 281-326.

Shostack, G.L. (1985), "Planning the Service Encounter", in: J.A. Czepiel, M.R. Solomon \& C.F. Surprenant (eds.), The Service Encounter, Lexington Books, Lexington, MA, 243254.

Slovic, P. \& D. MacPhillamy (1974), "Dimensional Commensurability and Cue Utilization in Comparative Judgment", Organizational Behavior and Human Performance, 11, 172 194.

Smith, P.B. \& 5. Schwartz (1997). "Values", in: J.W. Berry, M.H. Segall \& C. Kagitçibasi leds.). Handbook of Cross-Cultural Psychology. Vol. 3: Social Behavior and Applications, Allyn and Bacon, Boston, etc, 76-129.

Steenkamp, J.-B.E.M. \& H. Baumgartner" (1998), "Assessing Measurement Invariance in Cross-National Consumer Research", Journal of Consumer Research, 25, 78-90.

Steenkamp, J.B.E.M. \& H.C.M. van Trijp (1991), "The Use of LISREL in Validating Marketing Constructs", Intermational Joumal of Research in Marketing, 8 (4/November), 283-299.

Steenkamp, J.-B.E.M., F. ter Hofstede \& M. Wedel (1999), "A Cross-National Investigation into the Individual and National Cultural Antecedents of Consumer Innovativeness", Journal of Marketing, 63 (April), 55-69. 
Stelger, JH. (1990), "Structural model evaluation and modification: An interval estimation approach", Multivariate Behavioral Research, 25, 173-180.

Steinman, Ch. R. Deshpandé \& J.U. Farley (2000), "Beyond Market Orientation: When Customers and Suppliers Disagree" Journal of the Academy of Marketing Science, $28(1), 109-119$.

Sternthal, B. A.M. Tybout \& B.I. Calider (1987). "Confirmatory versus Comparative Approaches to Judging Theory Tests", Joumal of Consumer Research, 18 (June), 114125.

Stevens, 1. (1986), Applied Multivariate Statistics for the Social Sciences, Lawrence Erlbaum Associates, Hillisdale, NJ/London.

Styles, C. (1998), "Export Performance Measures in Australlia and the United Kingdom" " Journal of International Marketing, $6(3), 12-36$.

T

Tabachnik, B.G. \& L.5. Fidell (1996), Using Multivariate Statistics, Harper Collins College Publishers, New York.

Terpstra, V. \& R. Sarathy (1993), International Marketing, Harcourt Brace College Publishers, Fort Worth, etc.

Thompson, J. (1967), Organizations in Action, McGraw-Hill, New York.

Treviño, L.K., 1. Webster \& E.W. Stein (2000), "Making Connections: Complementary Influences on Communication Media Choices, Attitudes, and Use", Organization Science, $11(2), 163-182$.

Trillo, N.G. (1997), "Intercultural Computer-Mediated Communication", ComputerMediated Communication Magazine, $4(1)$.

Tucker, L.R. \& C. Lewis (1973), "The Reliability Coefficient for Maximum Likelihood Factor Analysis" "Psychometrika, 1-10.

$\bigcup$

Umesh, U.N., R.A. Peterson, M. MCCann-Nelson \& R. Vaidyanathan (1996) "Type IV Error in Marketing Research: The Investigation of ANOVA Interactions", Journal of the Academy of Marketing Science, 24 (1), 17-26.

Usunier, J.-C. (1996), Marketing Across Cultures, Prentice Hall, London, etc.

V

Vijver, F.J.R. van de \& K. Leung (1997), "Methods and Data Analysis of Comparative Research", in: J.W. Berry, Y.H. Poortinga \& J. Pandey (eds.), Handbook of Cross. Cultural Psychology, Vol. 1: Theory and Method, Allyn and Bacon, Boston, etc., 257. 300. 
W

Webster Jr., F.E. (1978), "Is Industrial Marketing Coming of Age?", in: T.V. Bonoma \& G. Zaltman (eds.), Review of Marketing. American Marketing Association, Chicago, 138-159.

Wetzels, M. K. de Ruyter \& M. van Birgelen (1998), "Marketing Service Relationships: The Role of Commitment", Journal of Business and Industrial Marketing. 13 (4/5), 406-423.

Wood, W. \& C.A. Kallgren (1988), "Communicator Attributes and Persuasion: Recipients" Access to Attitude-Relevant Information in Memory", Personality and Social Psychology Bulletin, 14, 172-182.

$Y$

Yamagishi, T. \& C.T. Hill (1981), "Adding versus Averaging Models Revisited: A Test of a Path-analytic Integration Model", Joumal of Personolity and Social Psychology, 41, $13-25$.

Yamagishi, T. \& M. Yamagishi (1994). "Trust and Communication in the United States and Japan", Motivation and Emation, 18, 129-165.

Vates, J.F., C.M. Jagacinski \& M.D. Faber (1978), "Evaluation of Partially Described Multiattribute Options", Organizational Behavior and Human Performance, 21, 240251.

\section{Z}

Zeithaml, V.A. (2000), "Service Quality, Profitability, and the Economic Worth of Customers: What We Know and What We Need to Learn" Journal of the Academy of Marketing Science, $28(1), 67-85$. 


\section{Appendix I}

\section{Modeling Hierarchical Relationships}

The modeling of both within-level and between-level relationships requires a simultaneous estimation of two models: one modeling relationships within each of the lower level units, and a second modeling how these relationships within units vary between units. This type of two level approaches defines hierarchical linear models (Bryk \& Raudenbush 1992; Raudenbush 1993). The level 1 (individualmevel) and level 2 (country-level) models for testing the hypotheses are formulated as follows:

Level 1:

$S A T_{i j}=\beta_{0 j}+\beta_{i j} E-S_{i j}+\beta_{2 j} C A L L_{i j}+\beta_{3 j} V I S I T_{i j}+e_{i j}$

Level 2:

$\beta_{0 j}=\gamma_{00}+\gamma_{01} P_{j}+\gamma_{02} 1 N D_{j}+\gamma_{09} M S_{j}+\gamma_{04} U A_{j}+u_{0 j}$

$\beta_{1 j}=\gamma_{10}+\gamma_{11} P_{j}+\gamma_{12} I N D_{j}+\gamma_{19} M A S_{j}+\gamma_{i, 4} U A_{j}+u_{1 j}$

$B_{21}=\gamma_{20}+\gamma_{21} P_{j}+\gamma_{22} \| N D_{j}+\gamma_{29} M A S_{j}+\gamma_{24} U A_{j}+u_{2 j}$

$\beta_{31}=\gamma_{30}+\gamma_{31} P D_{j}+\gamma_{32} I N D_{j}+\gamma_{93} M S_{j}+\gamma_{34} U A_{j}+u_{3 j}$

where $i$ stands for individuals; $j$ indicates countries; SAT refers to overall customer satisfaction; E-S, CALL and VISIT are electronic service, service call and service visit, respectively; PD, IND, MAS, and UA are power distance, indivildualism, masculinity, and uncertainty avoidance, respectively. Substituting Equations $\mathrm{A} 2 \mathrm{a}-\mathrm{A} 2 \mathrm{~d}$ in Equation $\mathrm{A} 1$ yields the following multilevel model (model C). which we estimated to test the hypotheses:

$$
\begin{aligned}
& S A T_{i j}=\gamma_{00}+\gamma_{10} E_{-} S_{i 1}+\gamma_{20} C A L L_{i j}+\gamma_{30} V_{1 S I T}+\gamma_{01} P D_{j}+\gamma_{02} I N D_{1}+\gamma_{03} M_{1 j} \\
& +\gamma_{04} U A_{j}+\gamma_{11} P_{j} \times E-S_{i j}+\gamma_{12} I N D_{j} \times E-S_{i j}+\gamma_{13} M A S_{j} \times E-S_{i j}
\end{aligned}
$$

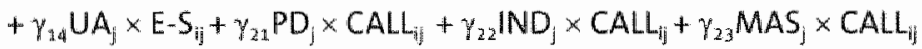

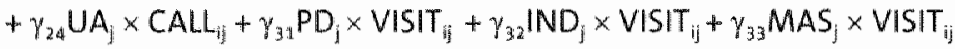

$$
\begin{aligned}
& +\gamma_{34} U A_{1} \times V I S I T_{i j}+\text { error term }
\end{aligned}
$$

The individual-level error term $e_{i j}$ is normally distributed with a mean of 0 and variance $\sigma^{2}$. The random effects $u_{q i}(q=0)$ are multivariate normally distributed over countries with an expected value of $0, \operatorname{var}\left(u_{q \mathrm{qj}}\right)=\tau_{q q}$, and $\operatorname{cov}\left(u_{q \mathrm{q}^{\prime}} u_{q \mathrm{qj}}\right)=\tau_{q q q^{\prime}}\left(q, q^{\prime}=0\right)$. $u_{\mathrm{qjj}}$ is the unique deviation of country $\mathrm{j}$ from the overall effect on the intercept $\left(\beta_{0 j}\right)$ while controlling for the country-level predictor variables. 
The level 1 submodel specifies the effects of the individual difference variables, whereas the level 2 submodel specifies the effects of country level variables. The level 1 predictors were centered within countries to guarantee numerical stability (Bryk \& Raudenbush 1992). Within country centering implies that the intercept $\beta_{0}$ is the mean level of SAT in country $j$. Within country centering has the important advantage that differences in mean levels between countries in the individual difference variables do not affect hypotheses testing. Furthermore, within country centering precludes multicollinearity between cross-level interactions and country level variables (Kreft \& de Leeuw 1998).

The coefficients $\left(\beta_{0 j}, \ldots, \beta_{3 j}\right)$ were specified as random coefficients; that is the coefficients were allowed to vary across countries. In theory, all $\beta_{\mathrm{a}} \mathrm{s}$ could be specified as random effects. However, from a statistical viewpoint such model is not recommendable, because it negatively affects model convergence and the stability of the parameter estimates (Bryk \& Raudenbush 1992). 


\section{Appendix II}

\section{Sample Scenario}

\section{(A sample scenario referring to complete information on both cross-national data equivalence and financial accountability of international service investments)}

Imagine yourself in the following decision-making situation:

A marketing research agency has conducted an international study among customers of several local operating companies to assess the perceived quality of various important service aspects and satisfaction with that service quality. You are being provided with an international research report in which the results of the study are presented. On the basis of this report you are expected to make decisions on (improving) your company's international service quality policy or assist in decisionmaking on this matter. When reading the report you find all the basic elements that you would normally expect to be present in a research report. These include issues such as information on research design, data collection methods, sampling techniques, sample composition, and results and recommendations regarding international customer service quality perceptions and satisfaction.

After browsing through the report, you are interested in more detailed information. Given the international character of the study, you would like to compare the various local operating companies to see which country scores the highest on customer satisfaction with the company's service quality. For this purpose, a table is included in the report that presents the overall customer satisfaction scores for every operating company. However, you wonder whether or not you can readily compare these scores. Due to differences in familiarity with research techniques and cultural differences regarding the service aspects that are considered to be important in the various countries, problems may arise when trying to compare the satisfaction scores. Different research procedures and conceptualizations of the service aspects under study may be effective in different cultures and countries. In the text accompanying the table, you read that several techniques have been applied by the research agency to establish equivalence in the data and that the satisfaction scores have been checked on this equivalence. This enables you to make quite reliable comparisons between the service performance of the various operating companies.

Additionally, you would like to know what the financial implications will be of decisions aimed at improving your company's international service quality level. In other words, you want to be convinced that investing in service aspects actually pays. 
off financially in the end. For this purpose, an appendix is included in the report that explicitly addresses this issue. On the basis of the recommendations given in the report, several cost-benefit scenarios are presented in the appendix. These provide you with useful insights on what financial costs international service quality improvement efforts will incur and what the financial benefits will be. 


\section{Nederlandse Samenvatting}

Binnen marketingkringen wordt meer en meer erkend dat kennis en informatie van doorslaggevende betekenis is woor het succes van organisaties. Op basis van marketing informatie kunnen strategische en tactische acties ondernomen worden die voordeel bieden voor zowel klanten als organisaties zelf. Het geven van een eenduidige definitie van informatie blijkt niet eenvoudig te zijn. Het is namelijk een complex concept dat uit meerdere facetten lijkt te bestaan. Wat echter wel duidelijk is, is dat het gebruik vain informatie om op de omgeving te kunnen reageren een centraal element in de definitie dient te zijn. Effectief gebruik van marketing informatie blijkt namelijk bepalend te zijn om meer marktgericht te opereren en de kans van slagen in een uiterst competitieve omgeving te vergroten. Dit geldt voor organisaties die op nationaal niveau opereren maar vooral ook voor bedrijven die hun diensten internationaal aanbieden. Dergelijke organisaties staan namelijk voor de dubbele uitdaging om effectief om te gaan met het risico en de onzekerheid waardoor diensten in het algemeen en internationale markten in het bijzonder gekenmerkt worden. Om te kumnen inspelen op de hoge mate van onzekerheid, onduidelijkheid en dymamiek in de internationale markt voor diensten, lijkt het beschikken over goede en betrouwbare internationale marketing informatie cruciaal te zijn.

Gezien het belang van informatie alsmede het feit dat er momenteel mede door ontwikkelingen in de informatie technologie (IT) en de internationale marktonderzoekpraktijk steeds meer een overvloed aan informatie lijkt te ontstaan is het cruciaal om meer aandacht te besteden aan de effectiviteit van informatie voor de internationalle marketing van diensten. Dit is dan ook de centrale missie van deze dissertatie. Hierbij gaan we ervan uit dat, om effectief te kunnen zijn, marketing informatie daadwerkelijk gebrukt dient te (gaan) worden door beshitvormers. Aangezien we informatie als een concept kunnen beschouwen dat uit meerdere facetten bestart, zal ons onderzoek naar de effectiviteit van informatie in een internationale dienstensetting ook meerdere perspectieven dienen te omvatten. Volgens onze visie zal namelijk voor een succesvolle vervulling van de centrale missie aandacht besteed dienen te worden aan de informatie zelf, alsmede de partijen die de informatie leveren en de partijen die deze dienen te (gaan) gebruiken.

De reden om de internationale dienstverlening alls onderzoeksdomein te kiezen komt woort uit het feit dat een groeiend aantal dienstverleners hun horizon lijken te verbreden. Verschillende factoren liggen hieraan ten grondslag. Het aambod van diensten kan nu de grenzen van nationale markten overschrijden, hetgeen vergemakkelijkt wordt door ontwikkelingen in de informatie- en communicatie 
technologie (ICT). Verzadiging van de thuismarkt kan op die manier vermeden worden. Verder wordt het door openstelling van buitenlandse markten steeds meer mogelijk voor dienstverleners om lokale vestigingen in buitenlandse locaties te openen en samenwerkingsverbanden met lokale partners aan te gaan. Toenemende internationalisering brengt vanzelfsprekend echter ook strategische en tactische problemen met zich mee die roepen om internationaal marktonderzoek. Zo zorgen culturele verschillen tussen landen en de onbekendheid met nieuwe markten er bijvoorbeeld voor dat meer aandacht geschonken dient te worden aan zaken als internationale promotie- en distributiestrategieën. Verder dienen de mate van internationalle klanttevredenheid en trends in de internationale omgeving nawkeurig. in het oog gehouden te worden en is informatie over de financiële gevolgen van investeringen ter bevordering van de internationalisering onontbeerlijk.

De centrale probleemstelling van deze dissertatie is als volgt geformuleerd:

\section{Welke factoren dragen bij tot de effectiviteit van marktonderzoekinformatie in internationale dienstverlenende organisaties?}

Zoals vermeld dienen voor de beantwoording van deze probleemstelling meerdere perspectieven ingenomen te worden. Deze perspectieven worden middels vier verschillende onderzoeksprojecten nader onderzocht. In de hoofdstukken 2 en 3 wordt volgens het informatie attribuut perspectief nader gekeken naar bepaalde karakteristieken van informatie en hoe deze gerelateerd zijn aan het gebruik van informatie door besluitvormers in internationale dienstverlenende organisaties. In hoofdstuk 4 wordt het informatie attribuut perspectief aangevuld worden met het relationele uitwisseling perspectief. De onderliggende gedachte hiervan is dat de levering van informatie in principe een relationele uitwisseling betreft tussen een dienstverlener (bijv. de marktonderzoeker) en een cliènt (bijv. de informatie gebruiker of besluitvormer). Dientengevolge zal er voor de bepaling van de effectiviteit van internationale marktonderzoekinformatie, naast de kwalliteit van de geleverde (onderzoek)dienst, ook rekening gehouden dienen te worden met interactie gerelateerde aspecten. Ons derde en laatste perspectief, het attitude perspectief geheten, komt aan bod in hoofdstuk 5 en richt zich op de interne attributie van gebruikers van informatie en het effect hiervan op de effectiviteit van internationale diensten informatie. Hierbij wordt gekeken naar het effect van de attitude van een gebruiker ten opzichte van het onderwerp van een onderzoek op bepaalde cognities en gedrag met betrekking tot dit onderwerp en de marktonderzoekinformatie hierover.

Meer in thet bijzonder wordt in hoofdstuk 2 een perspectief geintroduceerd dat erop gericht is om het effect van culturele variatie tussen landen op informatie over 
klantenevaluaties voor drie verschilfende vormen van dienstverlening te achterhalen. Aldus wordt gedemonstreerd hoe culturele achtergronden internationale marketing informatie over service prestaties kunnen nuanceren. Ten gevolge van technologische ontwikkelingen wordt het diensten portfolio van veel organisaties uitgebreid van de traditionele, persoonlijke dienstverlening naar tellefonische en elektronische dienstverlening. Meer in het bijzonder wordt de interactie onderzocht tussen de door klanten ervaren service prestatie en hun culturele achtergrond bij de totstandkoming van internationale marketing informatie over klanttevredenheid voor traditionele versus op technologie gebaseerde vormen van after-sales diensten. De resultaten van een empirisch onderzoek laten duidelijk zien dat in het geval van deze laatste vormen van dienstwerlening cultuur van invloed is op de relatie tussen kwaliteit van dienstwerlening en klanttevredenheid. Dientengevolge concluderen we dat cultuur verschillen een belangrijke factor vormen van effectieve marketing informatie voor internationale dienstverlening.

Ir hoofdstuk 3 vervolgen we het informatie attribuut perspectief. Terwijl uit hoofdstuk 2 gebleken is dat informatie over culturele verschillen relevant is zullen andere soorten wan informatie wellicht ook een rol spelen. Gegeven de aanzienlijke investeringen die veelal met de intermationalisering van diensten gemoeid zijn, lijkt het derhalve van belang om ook informatie over de financiële gevalgen van investeringen in de dienstverlening nader te beschouwen. Derhalve wordt er nu gekeken naar het relatieve effect van aan-dan wel afwezigheid van zowel culturele als financiêlle achtergrond informatie op de evaluatie en het gebruik van internationale marktonderzoekinformatie door besluitvormers in een dienstverlenende organisatie. Uit de resultaten van een experiment blijkt dat deze besluitvormers een gebrek aan unformatie niet zullen negeren. In plats hiervan zullen ze een strategie volgen waarbij ze de ontbrekende informatie zullen aanvullen middels inferenties en aannames. verder blijkt de mate van afwezigheid van informatie die relevant is voor de besluitvorming in internationale dienstverlening specifieke gevolgen te hebben voor bes/uitvormer tevredenheid, vertrouwen en intentie tot gebruik van de informatie.

In hoofdstuk 4 wordt dan vervolgens het informatie attribuut gezichtspunt aangevuld met de relationele uitwisseling benadering. Aangezien marktonderzoek gezien kan worden als een dienst die door een onderzoeker aan een gebruiker c.q. besluitvormer verleend wordt, zal voor de bestudering van effectiviteit van informatie voor internationalle dienstverlening het in overweging nemen van relationele aspecten nuttig kunnen zijn. Meer in het bijzonder wordt in dit hoofdstuk, naast informatie gerelateerde factoren, gekeken naar welke kenmerken van de onderzoeker het gebruik van informatie mogelijk beinvloeden. Aangezien ook academische onderzoekscentra zich in de afgelopen jaren meer en meer geprofileerd hebben als volwaardige 
onderzoekspartners wordt in dit onderzoeksproject expliciet gekeken naar dit type leveranciers van informatie. De resultaten van een tweejarig empirisch onderzoek suggereren dat tevredenheid van informatie gebruikers cruciaal is voor het genereren van vertrouwen en het bevorderen van informatie gebruik. In beide jaren blijken zowel inhoudelijke kwaliteit als eigenschappen wan de onderzoeker de voornaamste determinanten van tevredenheid te zijn. In een vroeg stadium van de relatie tussen onderzoeker en informatie gebruiker wordt informatie gebruik verder beïnwloed door de mate waarin de informatie politiek aanvaardbaar is. Wanneer de relatie zich verder ontwikkelt lijkt vooral de mate van vertrouwen in een onderzoeker een steeds. belangrijkere rol te gaan spelen, aangezien dit dan een direct effect op het gebruik van informatie blijkt te vertonen.

Na gekeken te hebben naar zowel de informatie zelf als de leverancier hiervan werpt het laatste onderzoeksproject dat centraal staat in hoofdstuk 5 een nadere blik op de informatie gebruiker. Door het attitude perspectief te volgen wordt nu getracht om intenties tot selectief gebruik van internationale klanttevredenheid informatie te voorspellen uit de sterkte van een attitude van een besluitvormer ten aanzien van dit onderwerp. De resultaten van een empirisch onderzoek laten zien dat twee dimensies van attitude sterkte, betrokkenheid ('commitment") en inbedding ('embeddedness') geheten, zowel direct als indirect bijdragen aan het selectief gebruik van marketing informatie in internationale dienstverlening. Indirecte effecten treden dan op via cognitieve processen en de selectieve beoordeling van tevredenheid gerelateerde informatie.

Om de centrale missie van deze dissertatie, het verkrijgen van een beter inzicht in de effectiviteit van marketing informatie in internationale dienstenmarketing, tot een succesvol einde te brengen wordt tenslotte in hoofdstuk 6 een uit meerdere facetten bestaande visie geformuleerd. Deze visie wordt in sterke mate gedreven door het feit dat internationale dienstverlening met name door voortschrijdende technologische ontwikkelingen steeds meeromvattend van aard zal zijn. ICT, Internet, intranet, extranet, etc. maken dat dienstverleners mationale en internationale klanten met steeds vernieuwende vormen van dienstverlening kunnen bereiken. Ondanks de veel gehoorde gedachte dat cultuur in een dergelijke setting er niet meer toe doet, blijkt uit deze dissertatie het tegendeel. Door de grotere onzekerheid en complexiteit van diensten, in combinatie met de crossculturele diversiteit van internationale markten, zal er een sterkere behoefte ontstaan aan cultureel verantwoorde onderzoekstechnieken die resulteren in effectievere informatie die beter bruikbaar is voor modern management van internationale diensten. Om de onzekerheid die met internationale dienstverlening verbonden is te reduceren dient deze informatie zo compleet mogelijk te zijn en, naast culturele aspecten, ook inzicht te verschaffen in 
bijvoorbeeld de financiële gevolgen wan investeringen in internationale dienstverlening. Daarnaast dienen voor de effectiviteit van informatie bepaalde attributen, zoals de inhoudelijke kwaliteit, alsmede relationele factoren, zoals het genereren van vertrouwen tussen informatie leverancier en gebruiker zeker niet onderschat te worden. Tevens zal een beter begrip van de informatie gebruiker en zijh/haar attitudes resulteren in informatie die effectiever gebruikt kan worden voor de internationale marketing van diensten. De dissertatie sluit tenslotte af met een perspectief op toekomstig onderzoek betreffende dit thema. 


\section{Curriculum Vitae}

Marcel J.H. van Birgelen was born on March 6, 1972 in Posterholt, the Netherlands. After completion of the secondary school "Bisschoppelijk College Schöndelin" in Roermond, The Netherlands), he studied Economics at the Faculty of Economics and Business Administration of the State University of Limburg, Maastricht. During these studies he specialized in Marketing and Marketing Research. In 1995 he started to work for the Department of Marketing \& Marketing Research as Research Assistant. In September 1996, after obtaining his Master's Degree, he joined the Department of Marketing \& Marketing Research as a lecturer. In November 1997, he became a Ph.D. Candidate and started working on his dissertation. His main research interests include international marketing research, international marketing intelligence effectiveness, intelligence utilization by decision-makers, services marketing, relationship marketing. and interactive/electronic marketing. The journals in which he has published include the Journal of Service Research the Journal of Business and Industrial Marketing, International Business Review, the European Journal of Marketing, Industrial Marketing Management, Total Quality Management, Maandblad voor Accountancy en Bedrijfseconomie, and Tijdschrift voor Marketing. Finally, he has also contributed various papers to conference proceedings and to the yearbook of the Dutch Association for Market Research and Information Management. 